\title{
Korrelationen zwischen struktureller Ordnung und elektrischen Transporteigenschaften in $\mathrm{CoFeB}|\mathrm{MgO}| \mathrm{CoFeB}$ Tunnelmagnetowiderstandselementen
}

\author{
Dissertation \\ zur Erlangung des Doktorgrades \\ der Mathematisch-Naturwissenschaftlichen Fakultäten \\ der Georg-August-Universität zu Göttingen
}

vorgelegt von

Gerrit Eilers

aus Westerstede

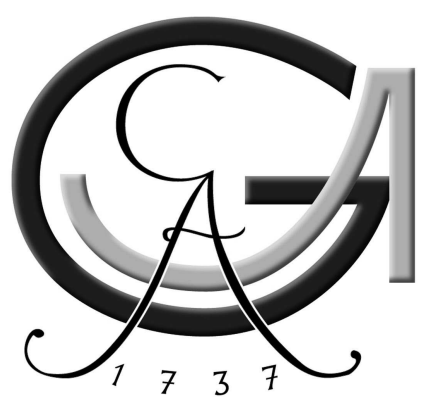

Göttingen 2009 
Referent:

Korreferent:

Tag der mündlichen Prüfung: 15.01.2010
Prof. Dr. M. Münzenberg

Prof. Dr. R. Kirchheim 


\section{Inhaltsverzeichnis}

$\begin{array}{lll}\mathbf{1} & \text { Einleitung } & 1\end{array}$

2 Theoretische Grundlagen $\mathbf{5}$

2.1 Bandstruktur und Austauschaufspaltung . . . . . . . . . . 5

2.2 Der Tunnelmagnetowiderstandseffekt . . . . . . . . . . . . 8

2.2 .1 Spinpolarisation: Modell nach Julliere. . . . . . . . . . 8

2.2 .2 Kohärentes Tunneln . . . . . . . . . . . . . . . 12

2.2 .3 Sauerstoff an der Grenzfläche ... . . . . . . . . 17

2.2 .4 Amorph-kristalline Elektroden . . . . . . . . . . . 20

2.2 .5 Sauerstofffehlstellen . . . . . . . . . . . . . . 23

3 Experimentelle Methoden 25

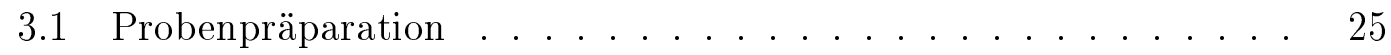

3.1.1 $\quad$ Elektronenstrahlverdampfen . . . . . . . . . . . . 25

3.1 .2 Magnetronsputterdeposition . . . . . . . . . . . . . . . . 26

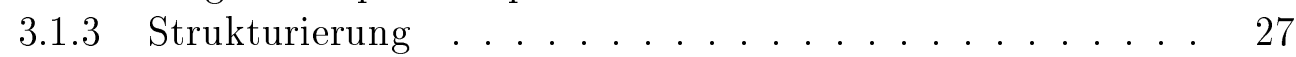

3.1 .4 Zielpräparation mittels FIB . . . . . . . . . . . . . . . 28

3.2 Untersuchungsmethoden . . . . . . . . . . . . . . . . . . . 30

3.2.1 Transmissionselektronenmikroskopie. . . . . . . . . . . 30

3.2 .2 Dreidimensionale tomographische Rekonstruktion aus STEM-Abbildungen $\ldots \ldots \ldots \ldots . \ldots . \ldots . \ldots 33$

$3.2 .3 \quad$ Mittelung von HRTEM Abbildungen . . . . . . . . . . . 34

3.2 .4 Multislice-Simulation gemittelter Abbildungen . . . . . . . 35

$3.2 .5 \quad$ Elektrische Transportmessungen $\ldots . . . . . . . . .39$

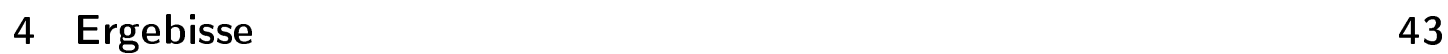

4.1 Modellsystem $\mathrm{MgO} / \mathrm{CoFeB}$. . . . . . . . . . . . . . . . . 43

$4.1 .1 \quad$ TEM-Untersuchungen auf der um-Skala . . . . . . . . . . 43

4.1.2 Hochaufgelöste Transmissionselektronenmikroskopie . . . . 44

4.1.3 Vergleich mit Simulationen . . . . . . . . . . . . . 46

4.2 Untersuchungen an realen Devices . . . . . . . . . . . . . . . . . . 52

4.2 .1 Teilkristallines Pseudo-Spinvalve. . . . . . . . . . . 53 
$4.2 .2 \quad$ Pseudo-Spinvalve mit thermisch zerstörtem TMR . . . . . 55

$4.2 .3 \quad$ Vergleich der Pseudo-Spinvalve-Systeme . . . . . . . . . 56

$4.2 .4 \quad$ Vollkristallines Pseudo-Spinvalve . . . . . . . . . . . . 60

$4.3 \quad$ Dielektrischer Durchbruch magnetischer Tunnelelemente . . . . . 65

4.3 .1 Überspannungszerstörte MTJs . . . . . . . . . . . . . . 66

$4.3 .2 \quad$ MTJs mit ultradünnen Barrieren . . . . . . . . . . . . . 68

$\begin{array}{lll}5 & \text { Zusammenfassung } & 75\end{array}$

\begin{tabular}{ll}
\hline Literatur & 83
\end{tabular} 


\section{Einleitung}

Der Tunnelmagnetowiderstandseffekt beschreibt das Phänomen, dass sich der Widerstand zwischen zwei ferromagnetischen Elektroden, die durch eine dünne isolierende Barriere getrennt sind, in Abhängigkeit von der relativen Ausrichtung der Magnetisierung der Elektroden zueinander, ändert. Dieser Effekt wurde zum ersten Mal 1975 von M. JulliÈRE 1 in einem Fe/Ge-O/Co-System experimentell gefunden, das eine Änderung des Tunnelwiderstands von $14 \%$ bei einer Temperatur von 4,2 K zeigte. Er erklärte diesen Widerstandseffekt mit einem Modell, das auf der zu Beginn der 70er Jahre von Meservey ${ }^{[2]}$ und Tedrow ${ }^{[3}$ nachgewiesenen Spinpolarisation des Tunnelstromes aus ferromagnetischen Elektroden beruht. Da es in der Folgezeit aber nicht gelang den Effekt auch bei Raumtemperatur nachzuweisen, blieb Jullières Entdeckung lange Zeit unbeachtet.

Mit der Entdeckung des Riesenmagnetowiderstands (Giant Magnetoresistance, GMR) durch A. FERT ${ }^{4}$ und P. GRÜNBERG ${ }^{[5}$ Ende der 80er Jahre, für den Sie 2007 mit dem Nobelpreis geehrt wurden, bekam auch die Forschung auf dem Gebiet des Tunnelmagnetowiderstands und des Spintransports allgemein neue Aufmerksamkeit. Der schnelle kommerzielle Erfolg des GMR, der schon kurz nach seiner Entdeckung Einzug in die Anwendung, zum Beispiel als Sensor in neuartigen Festplattenleseköpfen, fand, gab einem ganzen Gebiet der Forschung Auftrieb, das heute als „Spintronic“ bezeichnet wird. In der Folge wurde 1995 von MiYAzAKI ${ }^{[6]}$ und MOODERA ${ }^{[7}$ auch der TMR bei Raumtemperatur in magnetischen Tunnelelementen mit amorphen Aluminiumoxid-Barrieren und 3d-ÜbergangsmetallFerromagneten als Elektroden nachgewiesen. Durch weitere Optimierung dieses Systems konnte der TMR auf ca. $70 \%$ gesteigert werden, was zwar schon deutlich mehr war als die größte Widerstandsänderung, die mit dem GMR zu erreichen ist, für viele mögliche Anwendungen in der Spintronic aber immer noch zu wenig.

In 2001 erschienen zeitgleich zwei Arbeiten von BUTLER ET AL. ${ }^{8}$ sowie MATHON UND UMERSKI ${ }^{9}$, die beide unabhängig TMR-Werte von über $1000 \%$ für epitaktisch gewachsene Systeme mit kristalliner Magnesiumoxid (MgO)-Barriere vorhersagten, was auch zu der Bezeichnung Giant TMR für den Effekt in diesen Systemen führte. Die Vorhersagen gründen auf Berechnungen der komplexen Bandstruktur des $\mathrm{MgO}$ und der Annahme, dass Bloch-Zustände der Elektroden mit unterschiedlicher Symmetrie an passende abklingende Zustände in der Barriere koppeln. Daraus ergeben sich verschiedene Abklinglängen für Bloch-Zustände 
unterschiedlicher Symmetrie innerhalb der Barriere. Es zeigte sich, dass die $\mathrm{Zu}-$ stände mit $\Delta_{1}$-Symmetrie am effektivsten an die Barriere koppeln und hier am langsamsten abklingen. Zusammen mit der Tatsache, dass das $\Delta_{1}$-Band in Eisen bei der Fermi-Energie nur mit Majoritätsladungsträgern besetzt, also vollständig spinpolarisiert ist, folgt daraus ein sehr großer möglicher Tunnelmagnetowiderstand.

Die erste experimentelle Realisierung eines epitaktischen $\mathrm{Fe} / \mathrm{MgO} / \mathrm{FeCo}$ Tunnelkontakts gelangen BOWEN ET AL. ${ }^{10}$ ebenfalls in 2001. Sie erreichten allerdings nur einen TMR von $60 \%$ bei $30 \mathrm{~K}$ und $27 \%$ bei Raumtemperatur, der damit weit hinter den Prognosen von BUTLER ET AL. zurück blieb und nichtmal höher war als die besten Werte für Aluminiumoxid-Barrieren. Allerdings führten BowEN ET AL. keinen Auslagerungsschritt nach der Präparation ihrer Proben durch, was, wie spätere Beobachtungen anderer Gruppen zeigten, für das Erreichen hoher TMRWerte notwendig ist.

Wesentliche Fortschritte in Richtung auf die theoretisch vorhergesagten TMRWerte gab es erst 2004, zunächst durch YUASA ET AL.11, die in einem $\mathrm{Fe} / \mathrm{MgO} / \mathrm{Fe}$ Tunnelkontakt $88 \%$ TMR nachwiesen. Nur wenig später erschienen zwei Veröffentlichungen von YUASA ET AL. ${ }^{12}$ und PARKIN ET AL. ${ }^{13}$, die diesen Wert mehr als verdoppeln konnten. YUASA ET AL. ${ }^{[12]}$ konnten ihr mit MBE präpariertes System dabei soweit verbessern, dass sie einen TMR von $180 \%$ bei Raumtemperatur erreichten, während PARKIN ET AL. ${ }^{13}$ in einem gesputterten System mit Elektroden aus einer Eisen-Kobalt-Legierung einen TMR von bis zu $220 \%$ bei Raumtemperatur fanden.

Obwohl die bislang vorgestellten Ergebnisse wissenschaftlich große Fortschritte darstellen, ist ihre Bedeutung für eine großtechnische Anwendung gering. Nach den theoretischen Vorhersagen sind einkristalline ferromagnetische Elektroden notwendig, um hohe TMR-Werte erreichen zu können, weil eine wohldefinierte, saubere Grenzfläche benötigt wird, um eine perfekte Kopplung von BlochZuständen der Elektroden an abfallende Barrieren-Zustände zu ermöglichen. Um so größer war die Überraschung, als DJAYAPRAWIRA ET AL. 14 230\% TMR bei Raumtemperatur in $\mathrm{CoFeB} / \mathrm{MgO} / \mathrm{CoFeB}$-Tunnelelementen fanden. Für die Anwendungsaussichten stellte dies einen Durchbruch dar, weil die Struktur der CoFeB-Elektroden amorph ist, was eine Integration in bestehende Prozesse der Halbleiterindustrie deutlich erleichtert. Durch einen Auslagerungsschritt im Anschluss an die Schichtpräparation wird dabei erreicht, dass die ferromagnetischen Elektroden an der Grenzfläche zur MgO-Barriere lokal kristallisieren. Erstaunlicherweise zeigen diese Systeme mit amorphen ferromagnetischen Elektroden höhere TMR-Werte als die epitaktischen Systeme, wobei der aktuelle Rekord für Raumtemperatur bei $604 \%[15]$ liegt.

Die Hintergründe für diese unerwartet hohen TMR-Werte in Systemen mit 
amorphen ferromagnetischen Elektroden konnten später durch neuerliche theoretische Berechnungen aufgeklärt werden. So zeigten HEILIGER ET AL. ${ }^{16}$ 2007, dass schon wenige kristalline Lagen Eisen an der Grenzfläche zur MgO-Barriere ausreichen, um einen nahezu maximalen TMR zu erzeugen. Die experimentelle Bestimmung des quantitativen Zusammenhangs zwischen kristalliner Ordnung der ferromagnetischen Elektroden an der Grenzfläche zur MgO-Barriere und den elektrischen Transporteigenschaften der Tunnelelemente sind Gegenstand dieser Arbeit. Hierzu wurden aus bereits strukturierten Tunnelelementen, deren elektrische Transporteigenschaften so vermessen werden konnten, Querschnitte gefertigt und diese mit dem Transmissionselektronenmikroskop untersucht. Amorph/kristalline Grenzflächen spielen auch in anderen Bereichen eine wichtige Rolle, zum Beispiel die Grenzfläche zwischen kristallinem Silizium (Si) und amorphem Siliziumdioxid (SiO2) in MOS-Transistoren („Metal Oxide Semiconductor“), daher ist die Untersuchung der atomaren Struktur solcher Grenzflächen von großem Interesse. Dabei basiert die Strukturbestimmung mittels hochaufösender Transmissionselektronenmikroskopie auf dem quantitativen Vergleich von Intensitätsverläufen experimenteller und simulierter Abbildungen. Während die Simulation kristalliner Materialien einfach ist, gestaltet sie sich bei amorphen Materialien schwierig, da für die numerische Simulation die individuellen Atompositionen bekannt sein müssen. Einen Ausweg bietet hier ein von BORGARDT ET AL. 17] vorgeschlagenes Verfahren, welches die mittlere Verteilung des amorphen Materials in Grenzflächennähe statistisch durch eine dreidimensionale Verteilungsfunktion beschreibt. Dieses Verfahren wurde von K.THIEL $\stackrel{18}{ }$ auf die Grenzfläche zwischen kristallinem Silizium und amorphem Silizium bzw. amorphem Germanium angewand. Das dabei angewendete Verfahren zur Modellierung der Atomverteilung im amorphen Material wird im Folgenden in einer angepassten Form in dieser Arbeit für die Grenzfläche zwischen kristallinem $\mathrm{MgO}$ und amorphem $\mathrm{CoFeB}$ verwendet. 



\section{Theoretische Grundlagen}

In diesem Kapitel sollen die physikalischen Hintergründe zum Verständnis des Tunnelmagnetowiderstandes erläutert werden. Nach einer kurzen Einführung zum Ferromagnetismus wird hierzu ein einfaches Modell zum spinpolarisierten Tunneln vorgestellt. Darauf aufbauend wird auf die Besonderheiten beim kohärenten Tunneln eingegangen, bevor dann einige experimentell relevante Spezialfälle beschrieben werden.

\subsection{Bandstruktur und Austauschaufspaltung}

Ferromagnetismus beschreibt das Phänomen, dass sich die elementaren magnetischen Momente benachbarter Atome im Festkörper spontan, also ohne die Einwirkung eines äußeren Feldes, parallel ausrichten. In isolierten Atomen basiert das magnetische Moment auf den Elektronenspins und den Bahndrehimpulsen der Hüllenelektronen. Die parallele Ausrichtung zweier Spins liefert hierbei einen symmetrischen Beitrag zur gemeinsamen Wellenfunktion. Da das Pauli-Prinzip für Fermionen aber eine antisymmetrische Gesamtwellenfunktion fordert, führt dies zu einer antisymmetrischen Ortswellenfunktion, was einer Aufteilung der Elektronen auf verschiede Orbitale entspricht. Die dadurch erreichte stärkere räumliche Trennung der Elektronen führt zu einem Energiegewinn durch Absenkung der Coulombenergie, was als Austauschwechselwirkung bezeichnet wird. Eine unmittelbare Folge der Austauschwechselwirkung ist die 1.Hundsche Regel, welche besagt, dass bei der Verteilung der Hüllelektronen auf die möglichen Zustände im Atom der Gesamtspin maximiert wird. Im Allgemeinen haben also isolierte Atome, die nicht nur über komplett gefüllte oder leere Schalen verfügen, ein resultierendes magnetisches Moment, sofern sich nicht Spinmoment und Bahnmoment gegenseitig aufheben.

Betrachtet man einen Festkörper als durch die Annäherung freier Atome entstanden, so bewirkt die Überlappung der Wellenfunktionen ein Absenken und Aufspalten der elektronischen Zustände zu sogenannten Energiebändern. Wie im einzelnen Atom sind auch im Festkörper die möglichen Zustände der Elektronen durch eine Reihe von Quantenzahlen definiert, welche im Festkörper durch die Energie $E$, den Wellenvektor $\vec{k}$ und den Spin $\vec{s}$ repräsentiert werden. Die mögli- 

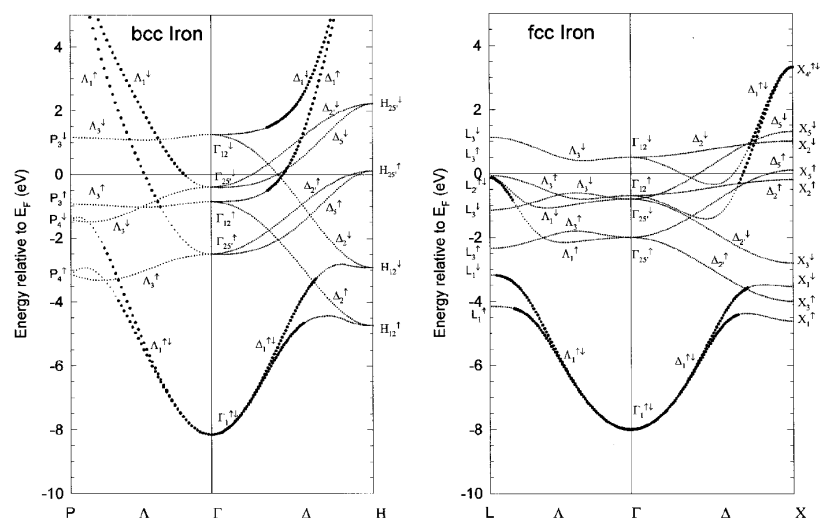

Abbildung 2.1: Bandstruktur von stabilem bcc-Eisen und einer metastabilen fcc-Phase, wobei letztere als dünner Film, wie hier auf Kupfer gewachsen, ferromagnetisch ist, sonst aber antiferromagnetisch (aus HIMPSEL ET AL. ${ }^{19}$ ).

chen, besetzbaren Zustände spannen im Impulsraum ( $\vec{k}$-Raum) meist kompliziert geformte $E(\vec{k})$ Flächen auf. Um sich einen Überblick über die Struktur der elektronischen Bänder zu verschaffen, werden Schnitte durch diese Energieflächen entlang ausgewählter Kristallrichtungen hoher Symmetrie aufgetragen. Es genügt die so erhaltene Dispersionsrelation für jede Richtung nur bis $k=\pi / a$ aufzutragen, weil nach dem Blochschen Theorem aus der Periodizität des Kristallgitters auch eine Periodizität in $E(\vec{k})$ folgt. Die experimentell ermittelte Bandstruktur für Eisen in bcc- und (metastabiler) fcc-Struktur ist in Abbildung 2.1 dargestellt.

Die d-Bandelektronen sind stärker lokalisiert als s- und p-Bandelektronen, daher erfahren sie eine stärkere Coulombabstoßung. Da auch im Festkörper jeder Zustand mit zwei Elektronen mit unterschiedlichem Spin besetzt werden kann, kann auch hier die Coulombenergie durch parallele Spinausrichtung abgesenkt werden. Auf der anderen Seite führt dies aber zu einer Erhöhung der kinetischen Energie, welche um so höher ist, je breiter das entsprechende Band ist. Ob diese Austauschaufspaltung von Vorteil ist, kann nur durch die Bilanzierung dieser Energien herausgefunden werden. Die folgende Bedingung, das sogenannte StonerKriterium ${ }^{22}$, dient daher als Indiz für das Vorhandensein von Ferromagnetismus.

$$
\tilde{D}\left(E_{f}\right) \cdot I>1 \text {. }
$$

Hierbei sind $\tilde{D}\left(E_{f}\right)$ die Zustandsdichte pro Spin und Atom an der Fermi-Energie und $I$ das Austauschintegral. Ferromagnetisches Verhalten ist also zu erwarten, 

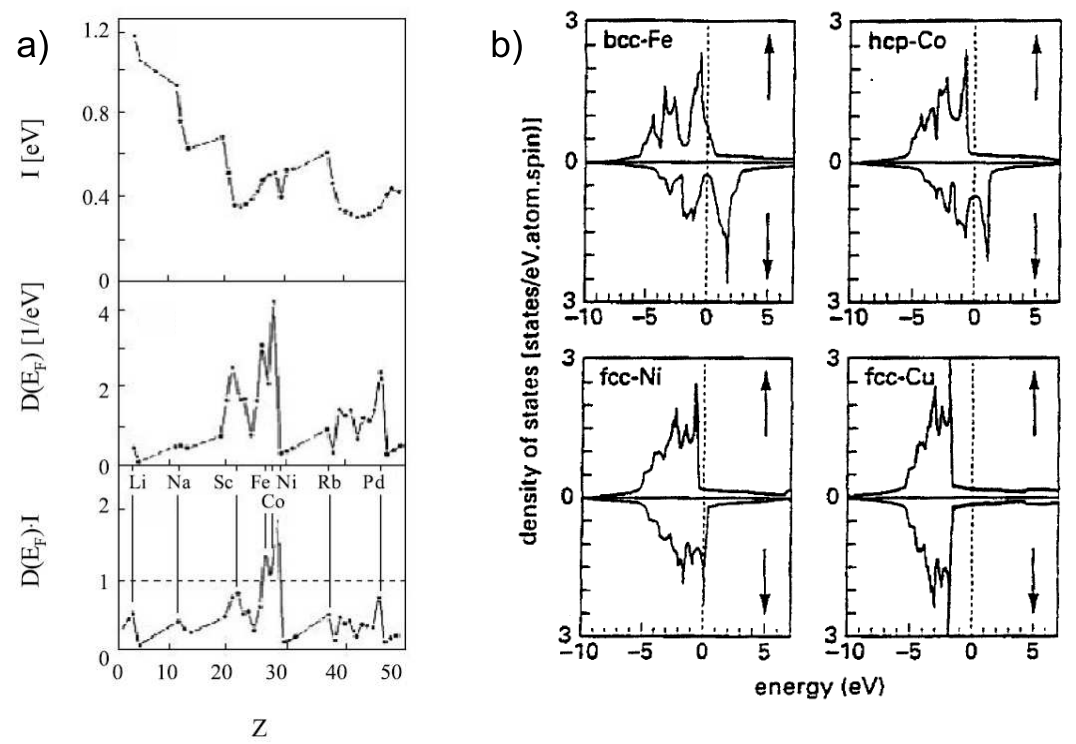

Abbildung 2.2: Stoner-Kriterium [20] (a) und Zustandsdichten verschiedener Übergangsmetallferromagneten (b) (aus MoOdERA ET AL. 21]).

wenn das Austauschintegral $I$ groß und die Zustandsdichte $D\left(E_{f}\right)$ in der nichtmagnetischen Berechnung an der Fermi-Energie $E_{f}$ hoch ist. Auch wenn die Zustandsdichte im Allgemeinen eine detailierte Struktur aufweist, kann man in erster Näherung annehmen, dass sie antiproportional zur Bandbreite ist. Für delokalisierte Elektronen ist die Bandbreite groß und die Zustandsdichte klein, während für stärker lokalisierte Elektronen das Band schmaler und damit die Zustandsdichte groß ist. Im Limit eines isolierten Atoms geht die Bandbreite gegen null, das Stoner-Kriterium ist immer erfüllt und das magnetische Moment ist maximal entsprechend der Hundschen Regel. Aus Abbildung 2.2 a ist zu entnehmen, dass die Bedingung bei Raumtemperatur für die 3d-Übergangsmetalle Eisen (Fe), Kobalt (Co) und Nickel (Ni) erfüllt ist.

Die stark lokalisierten d-Bandelektronen führen bei diesen Metallen also zur ferromagnetischen Austauschaufspaltung. Als Folge davon wird die Bandstruktur ohne Vorliegen eines äußeren Magnetfeldes in Subbänder mit unterschiedlicher Spinausrichtung aufgespalten. Die Energieabsenkung eines Subbandes im Bereich der Fermi-Energie führt zu einer spinabhängigen Anzahl der besetzten Zustände, die bevorzugte Spinausrichtung wird dabei als Majoritätsspin ( $\uparrow$ ), die entgegen- 
gesetzte als Minoritätsspin $(\downarrow)$ bezeichnet. In Abbildung $2.2 \mathrm{~b}$ sind die spinaufgespaltenen Zustandsdichten der drei Übergangsmetallferromagneten und die von Kupfer zum Vergleich dargestellt.

\subsection{Der Tunnelmagnetowiderstandseffekt}

In diesem Abschnitt wird zunächst ein simples Modell für spinpolarisiertes Tunneln eingeführt, welches sich ausschließlich auf die Spinpolarisation der tunnelnden Elektronen in den ferromagnetischen Elektroden als Ursache für Magnetowiderstandseffekte stützt. Im Folgenden werden die Besonderheiten des kohärenten Tunnelns in Systemen mit kristalliner Barriere, insbesondere MgO, erläutert. Danach soll der Einfluss der Grenzfläche zwischen Barriere und Ferromagnet sowie von Ordnungsstörung in der Nähe derselben auf den Tunnelmagnetowiderstand näher betrachtet werden. Dies führt dann zu Systemen mit amorphen ferromagnetischen Elektroden mit nur noch partieller Ordnung an der Grenzfläche. Abschließend wird dann Unordnung in der kristallinen Barriere anhand von Sauerstofffehlstellen behandelt.

\subsubsection{Spinpolarisation: Modell nach Julliere}

Mit der Entdeckung des spinpolarisierten Tunnelns durch Meservey Und TEDROw $1970^{[2] 3}$ wurde der Grundstein zu einem ganzen Feld in der aktuellen Forschung gelegt. Diese Experimente legten die Basis für das Verständnis des Tunnelmagnetowiderstandseffekts, wie auch für andere Phänomene spinpolarisierten Tunnelns. Sie konnten erstmals zeigen, dass der Strom tunnelnder Elektronen aus einer ferromagnetischen Elektrode heraus eine Spinpolarisation $P$ aufweist. Zur Messung der Spinpolarisation wurde supraleitendes Aluminium als Spindetektor benutzt. Die Quasiteilchen-Zustandsdichte eines dünnen, supraleitenden Aluminiumfilms unterliegt in einem großen magnetischen Feld der Zeemanaufspaltung. Durch Auswertung des Tunnelstromes von einem Ferromagneten mit seiner spinaufgespaltenen Zustandsdichte an der Fermi-Energie $E_{F}$ in einen spinaufgespaltenen supraleitenden Al-Film ergibt sich die Spinpolarisation der tunnelnden Elektronen zu:

$$
P=\frac{I_{\uparrow}-I_{\downarrow}}{I_{\uparrow}+I_{\downarrow}}
$$

Als wesentliches Ergebnis zeigen ihre Arbeiten, dass der Spin während des Tunnelprozesses erhalten bleibt, eine grundlegende Voraussetzung für die Existenz des 
Tunnelmagnetowiderstandseffekts. Für einen tieferen Einblick in das spinpolarisierte Tunneln sei auf den Review Artikel zu dem Thema von Meservey UnD TEDROW verwiesen.

Jullière ersetzte $1975^{1}$ den supraleitenden Spindetektor durch einen zweiten Ferromagneten und konnte zeigen, dass sich der Tunnelstrom in diesem Fe/Ge/Co System mit der relativen Orientierung der Magnetisierung in den Elektroden zueinander ändert. Anschaulich lässt sich dieser Tunnelmagnetowiderstandseffekt mit der spinaufgespaltenen Bandstruktur von Ferromagneten erklären. Jullière hat dafür ein einfaches Modell 1 zur Berechnung der Widerstandsänderung vorgeschlagen. Wie in Abschnitt 2.1 bereits beschrieben, werden die Elektronen mit der Spinorientierung parallel zur Magnetisierung als Majoritätselektronen bezeichnet, diejenigen mit entgegengesetzter Spinorientierung als Minoritätselektronen. Ist das d-Band, welches entscheidend für die Ausbildung des Ferromagnetismus ist, vollständig durch die Majoritätselektronen gefüllt, durch die Minoritätselektronen aber nur teilweise, wie im Falle von Nickel und Kobalt erfüllt (siehe Abbildung $2.2 \mathrm{~b}$ ), so haben die Majoritätselektronen an der Fermi-Kante eine geringere Zustandsdichte als die Minoritätselektronen, wie in Abbildung 2.3 schematisch dargestellt ist.

Parallel

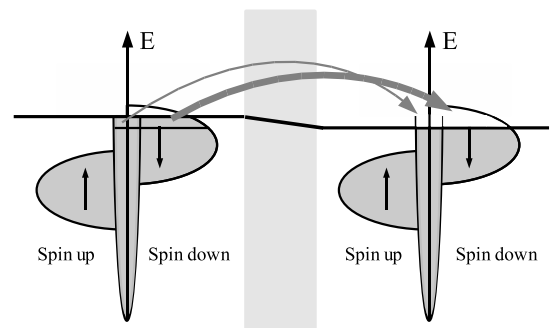

Antiparallel

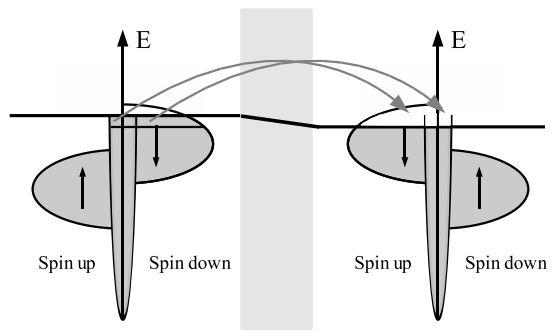

Abbildung 2.3: Schematische Darstellung des TMR-Effekts nach Jullière. Die Teilbänder der Majoritätselektronen sind jeweils mit einem $\uparrow$, die der Minoritätselektronen mit einem $\downarrow$ gekenzeichnet.

Im Falle einer parallelen Ausrichtung der Magnetisierungen der beiden beteiligten Ferromagneten sind die Minoritätselektronen der ersten Elektrode auch Minoritätselektronen in der zweiten. Da sie an der Fermi-Kante eine höhere $\mathrm{Zu}$ standsdichte haben als die Majoritätselektronen, können von dieser Spinsorte mehr Elektronen zum Tunnelstrom beitragen. Sie benötigen dafür aber auch freie Zustände in der zweiten Elektrode, in die sie hineintunneln können. Bei paralleler 
Ausrichtung der Magnetisierung stehen in der zweiten Elektrode ausreichend unbesetzte Zustände für die Minoritätselektronen zur Verfügung. Der zu erwartende Tunnelstrom ist also groß und wird im Wesentlichen von den Minoritätselektronen getragen. Im Falle einer antiparallelen Ausrichtung der Magnetisierungen sind die Minoritätselektronen der ersten Elektrode die Majoritätselektronen der zweiten. Es stehen zwar viele Minoritätselektronen in der ersten Elektrode in der Nähe der Fermi-Kante zur Verfügung, aber für sie sind nur wenige freie Zustände in der zweiten vorhanden. Für die Majoritätselektronen der ersten Elektrode hingegen sind genügend unbesetzte Zustände vorhanden, ihre Anzahl an der Fermi-Kante ist aber relativ gering. Bei paralleler Magnetisierungsausrichtung fließt also ein größerer Tunnelstrom als bei antiparalleler Ausrichtung. Nach dem Modell von Jullière leitet sich die Änderung des Kontaktwiderstands in magnetischen Tunnelelementen (JMR) ${ }^{1}$ von der Spinpolarisation folgendermaßen ab:

$$
J M R=\frac{\Delta R}{R}=\frac{R_{A P}-R_{P}}{R_{A P}}=\frac{2 P_{1} P_{2}}{1+P_{1} P_{2}} .
$$

Für $P_{1}$ und $P_{2}$ können die mit der Merservey-Tedrow-Methode bestimmten Polarisationen der ferromagnetischen Elektroden eingesetzt werden und so der nach Jullière zu erwartende JMR berechnet werden. Die gemessene Polarisation der Ferromagneten beträgt für Eisen $45 \%$, für Kobalt $42 \%$ [24 und für Nickel $33 \%$ [25. Die Definition von Jullière wird heute auch als pessimistischer Tunnelmagnetowiderstand bezeichnet. In der Literatur wird häufig eine abweichende Definition des optimistischen Tunnelmagnetowiderstands verwendet, der hier zur Unterscheidung mit TMR bezeichnet wird:

$$
T M R=\frac{\Delta R}{R}=\frac{R_{A P}-R_{P}}{R_{P}}=\frac{2 P_{1} P_{2}}{1-P_{1} P_{2}} .
$$

Jullières Formel kann den TMR unter Verwendung der von TEDROW UND MESERVEY ${ }^{23}$ experimentell bestimmten Spinpolarisationen erfolgreich vorhersagen. Allerdings lässt sich die so beobachtete Spinpolarisation nicht aus der Spinpolarisierten Zustandsdichte ableiten. Für Fe, Co und Ni würde man erwarten, dass der Tunnelstrom von den Minoritätselektronen dominiert wird und die Spinpolarisation somit negativ ist. Tatsächlich ist die experimentell bestimmte Spinpolarisation für alle Übergangsmetall-Ferromagneten positiv. Eine frühe Erklärung für diese Diskrepanz lieferte STEARNS ${ }^{26}$, indem sie anmerkte, dass die Transmissionswahrscheinlichkeit tunnelnder Elektronen von deren effektiver Masse $m_{e}^{*}$ abhängt, welche für unterschiedliche Bänder verschieden ist. So haben die relativ stark lokalisierten d-Elektronen, die in 3d-Ferromagneten den Großteil des

\footnotetext{
${ }^{1}$ engl. junction magnetoresistance
} 
magnetischen Moments tragen, eine hohe effektive Masse, weshalb ihre Zustände in der Barriere schnell abklingen. Demgegenüber haben mobile sp-hybridisierte Elektronen eine kleine effektive Masse und damit eine größere Abklinglänge in der Barriere. Sie schlug daher ein Modell mit separierten Kanälen für sp- und d-artige Elektronen vor, mit unterschiedlicher Spinpolarisation und Transmissionswahrscheinlichkeit für jeden Kanal. Bei hinreichend dicker Barriere (einige Monolagen) wird der Tunnelstrom also von sp-artigen Elektronen dominiert, welche in den 3d-Ferromagneten an der Fermi-Energie eine positive Spinpolarisation besitzen. Mit diesem einfachen Modell konnte Stearns die positive Spinpolarisation des Tunnelstromes trotz der negativen Spinpolarisation der Zustandsdichte erklären. Das Modell konnte 2004 von MÜNZENBERG ET AL. 27] experimentell bestätigt werden, indem die Spinpolarisation mit der Methode von Meservey und Tedrow für unterschiedlich dicke $\mathrm{Al}_{2} \mathrm{O}_{3}$ Barrieren vermessen wurde.

Eine weitere Ungenauigkeit des Modells von Jullière ist, dass die Eigenschaften der Barriere völlig außer Acht gelassen werden. So wurde zuerst von SLONCZEWSKI ${ }^{28}$ erkannt, dass der Tunnelkontakt als geschlossenes quantenmechanisches System zu behandeln ist und sich die Zustände der linken und rechten Elektrode in der Barriere überlagern. Hierzu modellierte er den Ferromagneten durch zwei einfache parabolische Bänder, welche in der Energie um eine Differenz $\Delta$ (Austauschaufspaltung) verschoben waren, und löste die Schrödinger Gleichung für tunnelnde Spin-up und Spin-down Elektronen durch eine rechteckige Barriere. Das prinzipielle Ergebnis seiner Berechnungen ist die zusätzliche Abhängigkeit der Polarisation des Tunnelstromes von der Barrierenhöhe $V_{b}$, welche den imaginären Wellenvektor $i \kappa$ in der Barriere beeinflusst. Mit $\hbar \kappa=\left[2 m\left(V_{b}-E_{F}\right]^{1 / 2}\right.$ wird die Polarisation $P$ zu:

$$
P=\frac{k^{\uparrow}-k^{\downarrow}}{k^{\uparrow}+k^{\downarrow}} \frac{\kappa^{2}-k^{\uparrow} k^{\downarrow}}{\kappa^{2}+k^{\uparrow} k^{\downarrow}}
$$

Hierbei sind $k^{\uparrow}, k^{\downarrow}$ die Fermi-Wellenvektoren im Spin-up bzw. im Spin-down Band. Unter der Annahme $k^{\uparrow} \propto D^{\uparrow}\left(E_{F}\right), k^{\downarrow} \propto D^{\downarrow}\left(E_{F}\right)$, welche für parabolische Bänder gültig ist, wird deutlich, dass der erste Faktor in Gleichung 2.5 der Polarisation nach der klassischen Tunneltheorie entspricht. Der zweite Faktor $A=\left(\kappa^{2}-k^{\uparrow} k^{\downarrow}\right) /\left(\kappa^{2}+k^{\uparrow} k^{\downarrow}\right)$ kommt neu hinzu. Da $\kappa$ Werte zwischen 0(niedrige Barriere) und $\infty$ (hohe Barriere) annehmen kann, gilt $-1<A<1$. Folglich entspricht Gleichung 2.5 für hohe Barrieren der Formel von Jullière, während sich für niedrige Barrieren gegenüber dieser sogar das Vorzeichen der Polarisation umkehren kann. In solchen Berechnungen ist es eine Vorraussetzung, dass der Elektronenimpuls parallel zur Barriere $k_{\|}$während des Tunnelns erhalten bleibt. Diese Erhaltung von $k_{\|}$, was auch als kohärentes Tunneln bezeichnet wird, ist in amorphen Barrieren wie $\mathrm{Al}_{2} \mathrm{O}_{3}$ im allgemeinen nicht erfüllt. In epitaktischen 
Systemen, zum Beispiel solchen mit kristalliner $M g O$ Barriere - welche in den folgenden Abschnitten behandelt werden - spielt das kohärente Tunneln hingegen eine entscheidene Rolle.

\subsubsection{Kohärentes Tunneln}

Das Wachstum von $\mathrm{MgO}$ auf Fe(001), sowie die Grenzfläche zwischen beiden, wurde in mehreren Studien untersucht. Dabei wurde sowohl für Eisen deponiert auf $\mathrm{MgO}(001)$ wie auch für den umgekehrten Fall von $\mathrm{MgO}$ deponiert auf Eisen(001) ein pseudomorphes Wachstum gefunden, bei dem die MgO[100] Richtung parallel zur Fe[110] Richtung ist und sich die Eisenatome über den Sauerstoffatomen des $\mathrm{MgO}$ anlagern, wie in Abbildung 2.4 gezeigt. Experimentell konnte gezeigt werden, dass bis zu sieben Monolagen $\mathrm{MgO}$ pseudomorph auf Fe(001) aufwachsen, bevor die geringe Gitterfehlpassung von $3,5 \% \mathrm{zu}$ Versetzungen führt 29 .
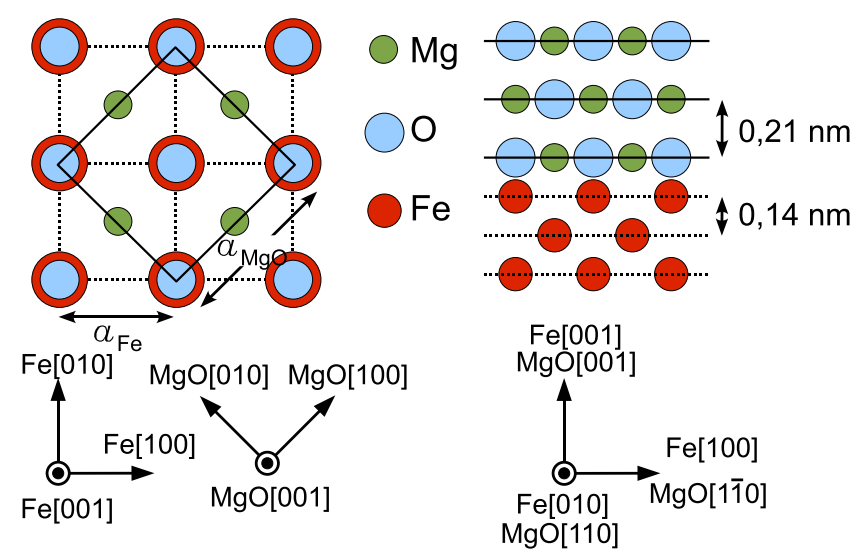

Abbildung 2.4: Epitaktisches Wachstum von bcc $\mathrm{Fe}(001)$ auf $\mathrm{MgO}(001)$. Nach YuAsA ET AL. $\stackrel{30}{.}$.

In einem epitaktisch aufgebauten Tunnelsystem aus $\mathrm{Fe}(001) / \mathrm{MgO}(001) / \mathrm{Fe}(001)$ lässt sich der Tunnelprozess nicht mehr durch das einfache Jullière Modell beschreiben. In diesem System spielt die effektive Kopplung von Bloch-Zuständen in der ferromagnetischen Elektrode an abklingende Zustände in der Barriere mit gleicher Symmetrie eine entscheidende Rolle. Es wurde zuerst von MAVROPOUlOS ET.AL. ${ }^{31}$ unter Berücksichtigung der komplexen Bandstruktur der isolierenden Barriere vorhergesagt, dass Elektronen mit $\Delta_{1}$ Symmetrie in Eisen die geringste Abklingrate in der Barriere haben. Obwohl sie sich im Wesentlichen 
mit halbleitenden Barrieren wie ZnSe oder Ge befassten, konnten sie die Bedeutung von epitaktischen $\mathrm{Fe} / \mathrm{MgO} / \mathrm{Fe}$ Tunnelsystemen für die Suche nach hohen Tunnelmagnetowiderständen als Erste vorhersagen.

Im Folgenden wurden 2001 für das System $\mathrm{Fe} / \mathrm{MgO} / \mathrm{Fe}$ von den Gruppen um BUTLER $^{[8]}$ sowie MATHON UND UMERSKI ${ }^{9]}$ unabhängig voneinander Berechnungen im Rahmen der Dichtefunktionaltheorie durchgeführt und ein hoher TMR von über $1000 \%$ theoretisch vorhergesagt. BUTLER ET AL. betrachteten dabei ein System aus mehreren Monolagen $\mathrm{MgO}(100)$ eingebettet zwischen ausgedehnten Eisenelektroden. Da bei der Präparation solcher Tunnelelemente mit einem kristallinen Eisensubstrat gestartet wird, kann man davon ausgehen, dass das $\mathrm{MgO}$ kompressiv verspannt auf diesem aufwächst. Für ihr Modell nahmen sie daher für die Eisenelektroden die experimentell bestimmte Gitterkonstante von $a_{\mathrm{Fe}}=2,866 \AA$, für den Eisen-Sauerstoff-Abstand $a_{\mathrm{Fe}-\mathrm{O}}=2,16 \AA$ und für die Gitterkonstante im $\mathrm{MgO} a_{\mathrm{MgO}}=\sqrt{2} a_{\mathrm{Fe}}=4,053 \AA$ an. Auf dieses System wird eine erweiterte Korringa-Kohn-Rostoker-Methode angewendet, welche den Vorteil bietet, dass keine Periodizität senkrecht zur Barriere erforderlich ist. Dies führt schließlich zur Landauer-Gleichung für die Leitfähigkeit:

$$
G=\frac{e^{2}}{h} \sum_{k_{\|}, j} T\left(k_{\|}, j\right) .
$$

Hierbei ist die Summation über j nötig, da es für jeden Wellenvektor parallel zur Barriere $k_{\|}$mehrere mögliche Bloch-Wellen gibt.

In Abbildung 2.5 sind die berechneten Tunnelwahrscheinlichkeiten als Funktion von $k_{\|}$für die verschiedenen Spinkanäle aufgetragen. Es ist leicht ersichtlich, dass für den Majoritätsspinkanal im Falle paralleler Magnetisierungsausrichtung ein deutliches Maximum der Transmissionswahrscheinlichkeit bei $k_{\|}=0$ auftritt. Die Schärfe dieses Maximums nimmt mit steigender Barrierendicke noch weiter zu, während die Verteilung der Transmissionswahrscheinlichkeit im Minoritätskanal und bei antiparalleler Magnetisierung deutlich komplizierter ist. Aufgrund der zweidimensionalen Periodizität des Gitters bleibt in epitaktischen Tunnelsystemen der Impulsanteil parallel zur Barriere $k_{\|}$erhalten. Die starke Konzentration der Transmissionswahrscheinlichkeit auf die Region um $k_{\|}=0$ ist daher vergleichbar mit dem Tunneln von freien Elektronen durch eine einfache Rechteckbarriere der Höhe $V_{b}$ und der Breite $d$. Für diesen Fall enthält die Transmissionswahrscheinlichkeit näherungsweise den Faktor $\exp (-2 d \kappa)$, wobei $\kappa^{2}=\left(2 m / \hbar^{2}\right)\left(V_{b}-E_{f}\right)+k_{\|}^{2}$ ist. In realen Systemen ist zwar die Variation der Transmission wesentlich komplizierter, dennoch macht die starke Konzentration der Transmission der Majoritätselektronen um $k_{\|}=0$ deutlich, dass das genaue Verständnis des Tunnelvorgangs im Fall $k_{\|}=0$ besonders wichtig ist. 
(a)

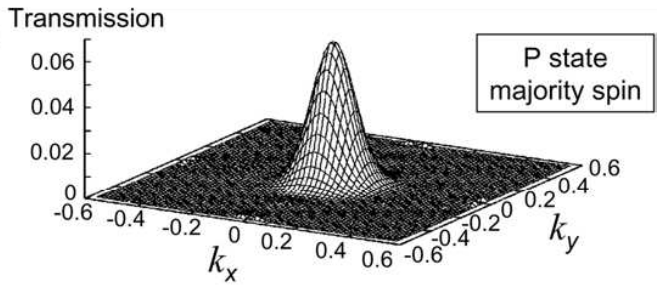

(b)
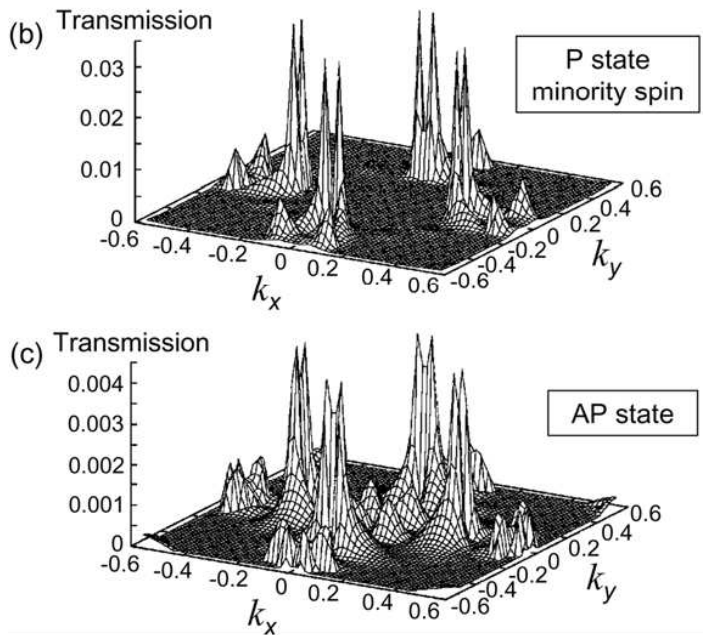

Abbildung 2.5: Tunnelwahscheinlichkeit in einem epitaktischen $\mathrm{Fe} / \mathrm{MgO} / \mathrm{Fe}$ Tunnelelement (4 Monolagen $\mathrm{MgO}$ ) als Funktion der Wellenvektorkomponenten parallel zur Barriere. (a) Majoritätsspinkanal in paralleler magnetischer Ausrichtung, (b) Minoritätsspinkanal in paralleler magnetischer Ausrichtung, (c) Antiparallele magnetische Ausrichtung. Aus Butler et Al. 8

In Abbildung 2.6 $\mathrm{a}$ ist die Bandstruktur für bcc Eisen in (001)-Richtung aufgetragen. Es gibt vier Bänder mit $k_{\|}=0$, die die Fermi-Energie schneiden. Die Bänder mit $\Delta_{5}$ und $\Delta_{2^{\prime}}$ Symmetrie haben für beide Spinrichtungen Zustände an der Fermi-Energie, wohingegen $\Delta_{1}$ Zustände nur für Majoritätsspins und $\Delta_{2} \mathrm{Zu}-$ stände nur für Minoritätsspins vorhanden sind. Im Gegensatz zur geringeren Gesamtspinpolarisation von Eisen sind die $\Delta_{1}$ und $\Delta_{2}$ Bänder bei der Fermi-Energie vollständig spinpolarisiert $(\mathrm{P}=1)$.

Es ist hilfreich, die Tunnelzustandsdichte für $k_{\|}=0$ der verschiedenen Energiebänder getrennt zu betrachten. Dabei ist die Tunnelzustandsdichte (TDOS) ${ }^{2}$ definiert als Zustandsdichte mit den Randbedingungen, das auf die von links einlaufenden Bloch-Zustände normiert wird. Abbildung 2.7 zeigt die Tunnelzustandsdichte für die verschiedenen $\mathrm{Fe}(100)$ Bloch-Zustände mit $k_{\|}=0$. In der linken Elektrode sind die einlaufenden und reflektierten Bloch-Wellen, in der Barriere deren Abfall und in der rechten Elektrode der transmittierte Anteil dargestellt. Da die Blochzustände von $\mathrm{Fe}(100)$ in der Barriere in der Bandlücke liegen, klingen sie hier mit der Abklingrate $\kappa$ exponentiell ab. Dabei können die Abklingraten aus der komplexen Bandstruktur des $\mathrm{MgO}$ hergeleitet werden und es zeigt sich,

\footnotetext{
${ }^{2}$ engl. tunneling density of states
} 

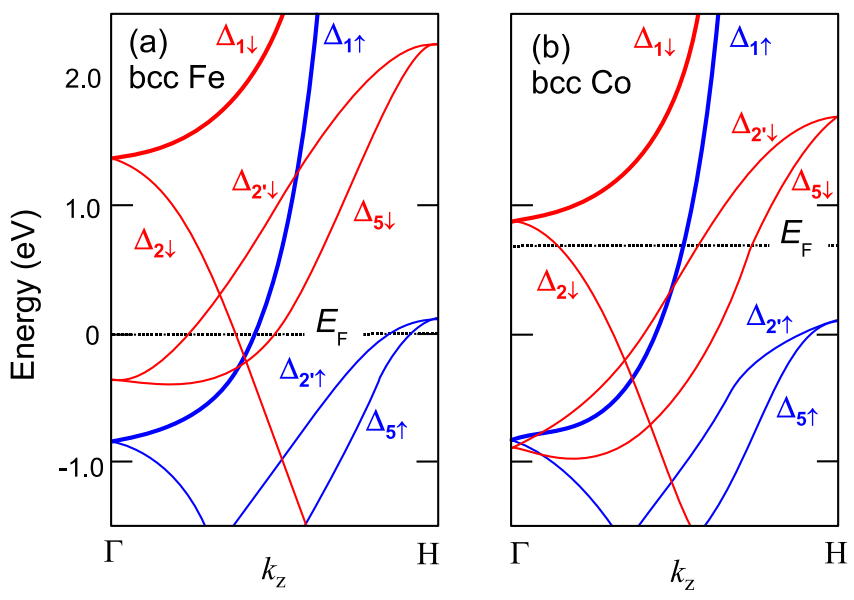

Abbildung 2.6: Bandstrukturen von bcc Fe und bcc Co entlang der [001]-Richtung für Majoritätselektronen (blau) und Minoritätselektronen (rot). Nach YUASA ET AL.

dass sie für Zustände unterschiedlicher Symmetrie verschieden sind. Dabei gilt:

$$
\kappa_{\Delta_{1}}<\kappa_{\Delta_{5}}<\kappa_{\Delta_{2^{\prime}}}<\kappa_{\Delta_{2}}
$$

Majoritäts-Bloch-Zustände mit $\Delta_{1}$ Symmetrie in der Eisenelektrode klingen in der MgO Barriere als Zustände mit derselben Symmetrie ab, gleiches gilt für Zustände mit $\Delta_{5}$ Symmetrie für beide Spinrichtungen. Die Zustände mit $\Delta_{2^{\prime}}$ Symmetrie beider Spinrichtungen klingen hingegen als Zustände mit $\Delta_{2}$ Symmetrie und die Minoritätszustände mit $\Delta_{2}$ Symmetrie als solche mit $\Delta_{2^{\prime}}$ Symmetrie in der Barriere ab. Diese scheinbare Symmetrieänderung hat ihre Ursache in der Verdrehung der kubischen Einheitszelle des $\mathrm{MgO}$ um $\pi / 4$ gegenüber der des Eisens.

Aus Abbildung 2.7 ist nun ersichtlich, dass bei paralleler Magnetisierung Majoritätselektronen mit $\Delta_{1}, \Delta_{5}$ und $\Delta_{2^{\prime}}$ Symmetrie am Tunnelprozess beteiligt sind. Solche mit $\Delta_{2^{\prime}}$ Symmetrie erreichen nur in sehr dünnen Barrieren die andere Elektrode und solche mit $\Delta_{5}$ Symmetrie klingen noch deutlich schneller ab als die mit $\Delta_{1}$ Symmetrie. Im Minoritätskanal gibt es keine $\Delta_{1}$ Zustände, dafür aber welche mit $\Delta_{2}$ Symmetrie, die aber schneller als die $\Delta_{5}$ Zustände abklingen. Bei paralleler Konfiguration wird der Tunnelstrom also maßgeblich von Elektronen mit $\Delta_{1}$ Symmetrie getragen. Bei antiparalleler Magnetisierung gibt es im Majoritätskanal wieder Zustände mit $\Delta_{1}, \Delta_{5}$ und $\Delta_{2^{\prime}}$ Symmetrie, für die $\Delta_{1}$ Elektronen gibt es aber in der zweiten Elektrode keine besetzbaren Zustände, da sie hier Minoritätsladungsträger sind. Die $\Delta_{1}$ Zustände klingen also in der rechten Elektrode 

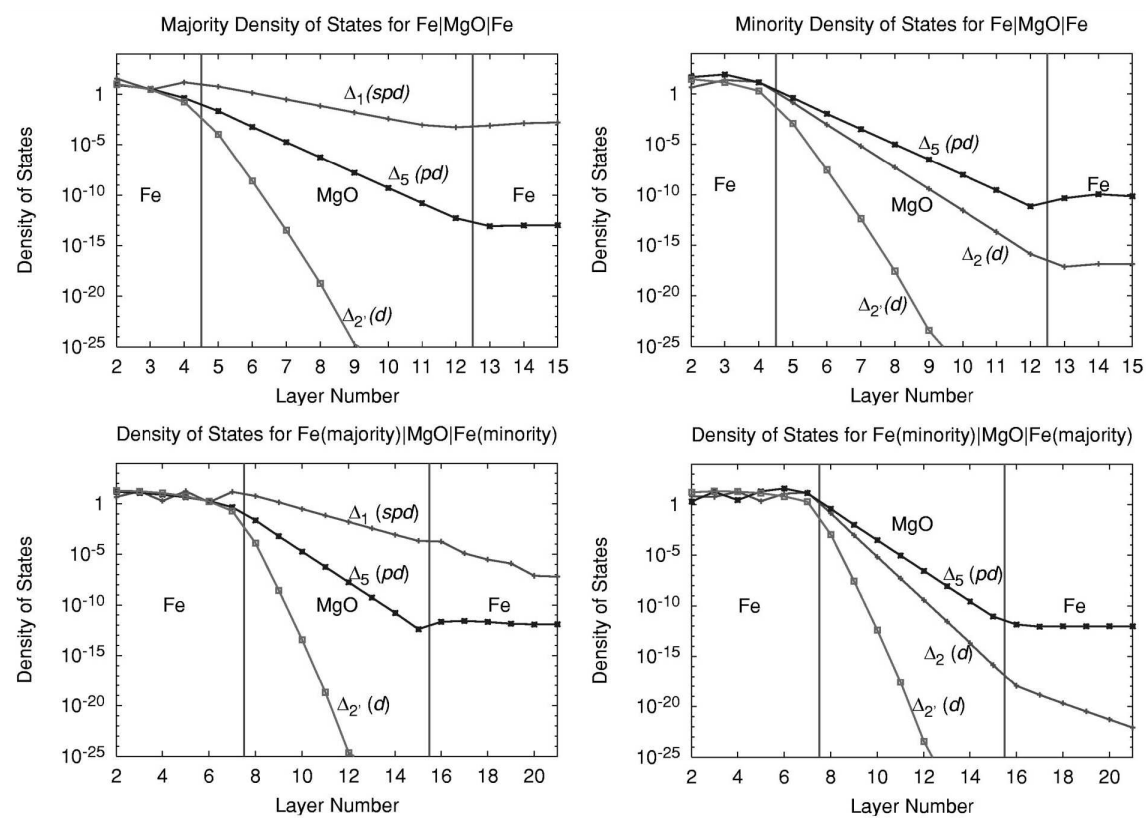

Abbildung 2.7: Tunnelzustandsdichte für $k_{\|}=0$ in $\mathrm{Fe}(100)|8 \mathrm{MgO}| \mathrm{Fe}(100)$. Gezeigt sind parallele (oben) und antiparallele (unten) Magnetisierungsausrichtung, jeweils der Majoritätsspinkanal (links) und Minoritätsspinkanal (rechts). Aus Butler ET AL. 8

weiter ab und werden vollständig reflektiert. Im Minoritätskanal erreichen wieder Elektronen mit $\Delta_{5}$ und $\Delta_{2}$ Symmetrie die andere Elektrode aber auch die $\Delta_{2}$ Elektronen werden vollständig reflektiert. Bei hinreichend dicker Barriere dominiert das vollständig spinpolarisierte $\Delta_{1}$ Band den Tunnelprozess und begründet so die hohen erzielbaren Werte des TMR.

Später wurden diese Berechnungen z.B. von ZHANG UND BUTLER auf andere epitaktische Systeme wie bcc $\mathrm{Co}(100) / \mathrm{MgO}(100) / \mathrm{bcc} \mathrm{Co}(100)$ und Fe$\mathrm{Co}(100) / \mathrm{MgO}(100) / \mathrm{FeCo}(100)$ übertragen ${ }^{32}$. Für diese magnetischen Tunnelsysteme konnte ein um ein Mehrfaches höherer TMR vorhergesagt werden als vorher für das System $\mathrm{Fe} / \mathrm{MgO} / \mathrm{Fe}$. Dies liegt vor allem daran, dass in bcc Co im Majoritätskanal ausschließlich das $\Delta_{1}$ Band die Fermi-Energie schneidet (siehe Abbildung 2.6b). Im Minoritätskanal schneiden hingegen nur die Bänder mit $\Delta_{2}, \Delta_{5}$ und $\Delta_{2^{\prime}}$ Symmetrie die Fermi-Energie, das heißt alle Bänder sind für $k_{\|}=0$ vollständig spinpolarisiert und bei antiparalleler Magnetisierung werden alle einlaufenden Blochwellen reflektiert. 


\section{Tunneln durch Grenzflächenresonanzzustände}

Obwohl sich die $k_{\|}$-Abhängigkeit der Transmission im Majoritätsspinkanal noch annähernd mit dem Modell freier Elektronen und einer einfachen Rechteckbarriere beschreiben lässt, sieht die Situation im Minoritätsspinkanal (vergl. Abbildung 2.5 völlig anders aus. Die komplizierte Struktur der Transmission mit ihren sehr scharfen Maxima in der zweidimensionalen Brillouinzone hat ihre Ursache in einem Zusammenspiel von Grenzflächenresonanzzuständen, der $k_{\|}$-Abhängigkeit der Abklingrate für die Wellenfunktionen im $\mathrm{MgO}$ und der Symmetrie der Minoritäts-Bloch-Zustände im Eisen. Die Minoritätszustandsdichte auf der Grenzflächenlage des Eisens weist vier Maxima auf, jeweils zwei für $k_{x}=0, k_{y}= \pm 0,4$ inversen Bohrradien bzw. $k_{y}=0, k_{x}= \pm 0,4$ inversen Bohrradien. Die Tunnelzustandsdichte des $\mathrm{MgO}$ an den Stellen der Maxima der Grenzflächenzustandsdichte des Eisens weist eine sehr kurze Abklinglänge auf, da an diesen Punkten keine s-artigen Blochzustände existieren. Ein kleines Stück neben den Achsen $k_{x}=0$ bzw. $k_{y}=0$ in der zweidimensionalen Brillouinzone ist die Abklingrate der Tunnelzustandsdichte im MgO schon sehr viel kleiner. Diese beiden Effekte führen im Zusammenspiel zu den scharfen Doppelpeaks der Transmissionswahrscheinlichkeit für den Minoritätskanal, wie sie in Abbildung 2.5 gezeigt sind. Diese sogenannten hot spots werden sowohl von BUTLER ET AL. als auch von MATHON UND UMERSKI beschrieben und belegen die große Bedeutung der Grenzfläche zwischen Eisen und MgO für das Erreichen hoher TMR Werte.

\subsubsection{Sauerstoff an der Grenzfläche}

In der Folge der Vorhersagen für extrem große TMR Werte versuchten mehrere Gruppen ein solches System herzustellen. Zu den Ersten gehörten WuLFHEKEL ET AL. 29] und KlaUA ET AL. ${ }^{33}$, die MgO Tunnelbarrieren auf einkristallinen Eisensubstraten präparierten und die Struktur sowie die Tunnelcharakteristik im Detail mittels Rastertunnelmikroskopie untersuchten. Dabei konnten sie das pseudomorphe Wachstum von $\mathrm{MgO}$ auf $\mathrm{Fe}(001)$ und die guten Tunneleigenschaften der MgO Barriere nachweisen, jedoch mangels Messmöglichkeit noch keinen TMR. Als erste fanden schließlich BOWEN ET AL. 10] einen TMR von $27 \%$ bei Raumtemperatur in einem epitaktischen System von $\mathrm{Fe} / \mathrm{MgO} / \mathrm{FeCo}$ auf einem GaAs Substrat mit MgO Pufferschicht. Einen etwas größeren TMR von $67 \%$ fanden 2003 FAURE-Vincent ET AL. 34 in einem epitaktischen $\mathrm{Fe} / \mathrm{MgO} / \mathrm{Fe}$ Tunnelsystem mit deutlich dickeren Barrieren. Es bleibt festzuhalten, dass die bis hierher erzielten Magnetowiderstände in Systemen mit $\mathrm{MgO}$ Tunnelbarriere jene für amorphe $\mathrm{Al}_{2} \mathrm{O}_{3}$ Barrieren nicht überstiegen und daher noch im Einklang mit Jullières Modell sind und kein Indiz für kohärentes Tunneln liefern. In allen theoretischen Be- 
trachtungen im vorigen Abschnitt wurde immer perfekt epitaktisches Wachstum und eine saubere Grenzfläche vorausgesetzt. Eine solche perfekte Probenstruktur bereitet in der Praxis natürliche einige präparative Schwierigkeiten.

MEYERHEIM ET AL. untersuchten als erste gezielt das Wachstum von MgO auf Fe(100) mittels oberflächensensitiver Röntgenbeugung (SXRD) ${ }^{35}$. Dabei beobachteten sie eine teilweise Oxidation der obersten Eisenlage, wobei in etwa $60 \%$ der Oktaederlücken der Fe(100) Oberfläche ein Sauerstoffatom eingebaut wird, wie in Abbildung 2.8 gezeigt. Dies legt die Vermutung nahe, dass die FeOGrenzschicht die effektive Kopplung der Blochzustände des Eisens an die abfallenden Zustände des MgOs behindert. Nach diesen Erkenntnissen präsentierten ZHANG ET AL. neue theoretische Berechnungen über das spinabhängige Tunneln unter Berücksichtigung einer teilweise oxidierten Eisenzwischenschicht ${ }^{[36}$. Sie fanden dabei einen deutlich reduzierten TMR von $76 \%$ für eine vollständig oxidierte Eisenoberfläche, was gut mit den bis dahin erzielten experimentellen Ergebnissen übereinstimmte.

(a)

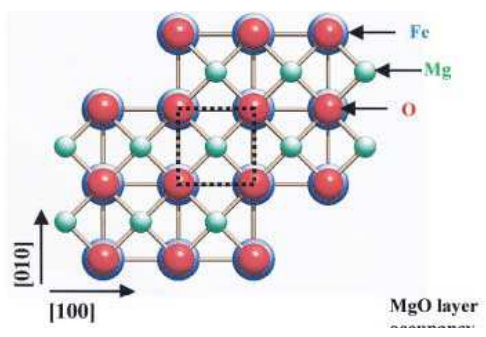

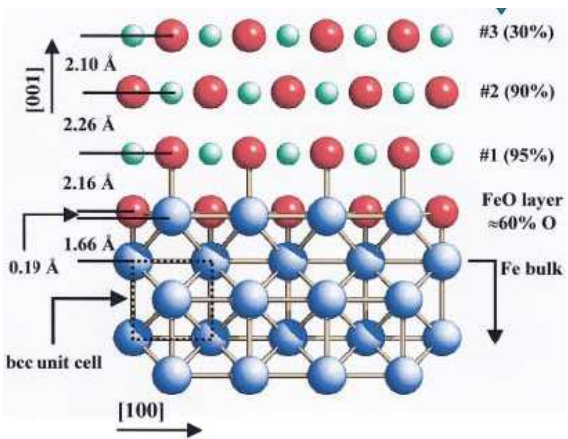

Abbildung 2.8: Grenzfläche $\mathrm{MgO} / \mathrm{Fe}(001)$ in Draufsicht (a). Einbau einer $\mathrm{FeO}$ zwischenschicht (b). Aus MeYerheim ET AL. ${ }^{35}$.

Da die $\Delta_{1}$-Zustände des Majoritätsspinkanals den Hauptteil des Tunnelstromes tragen, ist die Kopplung dieser Zustände an die benachbarte MgO Lage ein kritischer Faktor für den TMR. In Abbildung 2.9 ist daher die partielle Zustandsdichte dieses $\Delta_{1}$-Zustands an der Fermi-Energie in der Nähe der Grenzfläche dargestellt. Durch die zusätzlichen Bindungen zwischen den Eisen- und Sauerstoffatomen in der FeO-Zwischenschicht kommt es zu einer Ladungsverschiebung und gleichzeitiger Schwächung der Bindung der Eisenatome der Grenzfläche an die Sauerstoffatome der ersten MgO-Lage. In den Lagen, in denen Sauerstoffatome vorhanden sind, fließt der Tunnelstrom im Wesentlichen über diese. Hinzu kommt der Umstand, dass sich die Symmetrie der Wellenfunktion auf den Sauerstoffatomen 
in der FeO-Lage von der auf den Sauerstoffatomen im $\mathrm{MgO}$ unterscheidet. Dies führt zu einer ineffektiven Kopplung des $\Delta_{1}$-Zustands des Eisens an das MgO. Eine FeO-Zwischenschicht kann so den Tunnelstrom in paralleler Konfiguration um mehr als eine Größenordnung absenken, während gleichzeitig der Tunnelstrom in antiparalleler Konfiguration nahezu unbeeinflusst bleibt, was zu einer drastischen Abnahme des TMR führt.

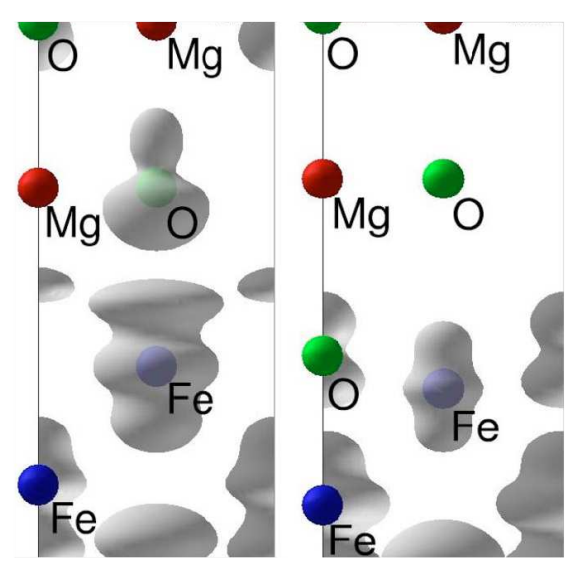

Abbildung 2.9: Partielle Zusstandsdichte der $\Delta_{1}$-Zustände an der Fermi-Energie in der Nähe einer idealen (a) und einer oxidierten $\mathrm{Fe}(001) / \mathrm{MgO}(001)$ Grenzfläche. Aus ZHANG ET AL. $\stackrel{36}{ }$.

Anfang 2004 erreichten YUASA ET AL.11 erstmals einen TMR von $88 \%$ bei Raumtemperatur in epitaktischen $\mathrm{Fe} / \mathrm{MgO} / \mathrm{Fe}$ Tunnelelementen. Dabei zeigte der TMR ein stark asymmetrisches Verhalten bezogen auf die Stromrichtung, für positive Spannung der oberen Elektrode wurde eine Halbwertsspannung von 1250 $\mathrm{mV}$ gemessen, was dreimal so groß ist wie der für $\mathrm{Al}_{2} \mathrm{O}_{3}$ Barrieren bekannte Wert. Kurze Zeit später erschienen zwei Veröffentlichungen, von YUASA ET AL. 12] und von PARKIN ET AL. ${ }^{13}$, die einen nochmals verdoppelten TMR zeigten. Auch hier trat die starke Asymmetrie des TMR auf, was auf eine schlechtere untere Grenzfläche gegenüber der oberen hindeutete. HEILIGER ET AL. ${ }^{[37}$ präsentierten daraufhin 2005 theoretische Berechnungen für drei verschiedene Modellsysteme, zum einen perfekte Grenzflächen zum anderen symmetrisch auftretende FeO Zwischenschichten und als drittes ein System mit nur einer FeO-Grenzschicht und einer perfekten Grenzfläche. Für alle Systeme berechneten sie die Bias-Abhängigkeit des TMR und konnten so die beobachtete Asymmetrie mit den FeO-Grenzschichten in Zusammenhang bringen. Sie zeigten sogar einen spannungsabhängigen Wechsel 
von positivem zu negativem TMR, was sie auf den Einfluss resonanter Grenzflächenzustände im Minoritätskanal zurückführten. Diese Ergebnisse deckten sich

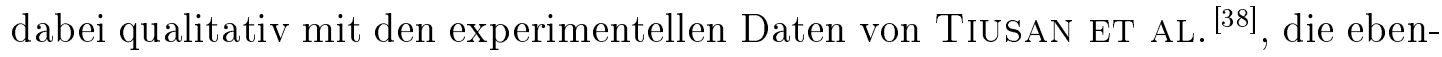
falls zeigen konnten, dass diese resonanten Zustände nur auftreten, wenn sie an bulk-Zustände in ausgedehnten Eisenelektroden koppeln können. Bei sehr dünnen Elektrodenschichten tritt daher der Vorzeichenwechsel im TMR nicht auf.

Den Einfluss der Wachstumsbedingungen auf die Eigenschaften der oberen Grenzfläche untersuchten Tusche ET AL. ${ }^{39}$, indem sie die erste Atomlage der oberen Eisenelektrode in einer kontrollierten Sauerstoffatmosphäre aufwuchsen. Dabei untersuchten sie ihre Schichten ebenfalls mit oberflächensensitiver Röntgenbeugung und konnten zeigen, dass sich durch einen gezielten SauerstoffHintergrunddruck auch an der oberen Grenzfläche eine FeO-Zwischenschicht erzeugen lässt, wodurch sich ein vollständig symmetrischer Schichtaufbau erreichen lässt. Sie führten für die so bestimmten Probengeometrien auch ab initio Rechnungen zum TMR in Abhängigkeit der Barrierendicke durch und fanden, dass im Falle symmetrischer FeO-Zwischenschichten ein TMR von mehreren $1000 \%$ zu erwarten ist, während sich für das asymmetrische System für dünne Barrieren von bis zu 6 Monolagen ein negativer und für dickere ein kleiner positiver TMR ergibt, was in guter Übereinstimmung mit den Untersuchungen von Heiliger ET AL. ${ }^{37}$ steht. Aus den Berechnungen von Heiliger ergibt sich auch ein qualitativer Unterschied zwischen den beiden symmetrischen Systemen. So ist der TMR in perfekt epitaktischen Systemen ohne FeO an der Grenzfläche nahezu unabhängig von der Bias-Spannung, während der TMR in Systemen mit symmetrischer FeO Zwischenschicht kontinuierlich mit der angelegten bias-Spannung abnimmt, wie es in experimentellen Daten immer beobachtet werden kann.

2006 veröffentlichten YU UND KIM ${ }^{[0]}$ ab initio Berechnungen zum Wachstum ultradünner $\mathrm{MgO}$ Schichten auf $\mathrm{Fe}(100)$. Dabei zeigten die Berechnungen der freien Energie, dass sich unter Mg-reichen Bedingungen die Bildung von FeO unterbinden lässt. Unter sauerstoffreichen Bedingungen hingegen ist die Bildung einer FeO Zwischenschicht bevorzugt. Das bedeutet, dass bei geschickter Wahl der Wachstumsparameter die Herstellung epitaktischer Fe(100)/MgO(100)/Fe(100) Tunnelelemente mit vollständig symmetrischem Aufbau, sowohl mit als auch ohne FeO-Zwischenschicht, und hohem TMR möglich ist.

\subsubsection{Amorph-kristalline Elektroden}

Ein entscheidener Schritt im Hinblick auf technische Anwendungen wurde erreicht, als man erkannte, dass sich auch in nicht epitaktischen Systemen bestehend aus amorphen $\mathrm{CoFeB}$-Elektronen mit kristalliner MgO-Barriere und anschließendem Auslagerungsschritt ein hoher TMR erreichen lässt. Der große Vorteil gegenüber 
den epitaktischen Systemen hierbei ist, dass sich solche Tunnelelemente wegen der einfach herzustellenden amorphen Elektrodenschichten problemlos in bestehende technische Prozesse der Halbleiterindustrie integrieren ließen. Zur allgemeinen Überraschung fanden DJAYAPRAWIRA ET AL. 14 Anfang 2005 einen TMR von $230 \%$ bei Raumtemperatur in einem solchen System. Dabei hat sich gezeigt, dass das MgO texturiert in (001)-Richtung auf der amorphen CoFeB-Schicht aufwächst und diese Struktur während der Auslagerung auf die CoFeB-Elektroden überträgt. YUASA ET AL. untersuchten das Wachstum von MgO auf CoFeB mittels RHEED und fanden ein anfangs amorphes Wachstum, welches bei einer Schichtdicke von 1 $\mathrm{nm}$ in ein texturiertes übergeht. Transmissionselektronenmikroskopische Aufnahmen eines solchen Systems noch vor dem Auslagerungsschritt zeigten eine bereits völlig kristalline Barriere. Die anfangs teilweise amorphe $\mathrm{MgO}$ Barriere ist also leicht zu kristallisieren, möglicherweise durch unbeabsichtigte Temperatursteigerung während der Deposition oder während der Präparation der TEM-Lamelle.
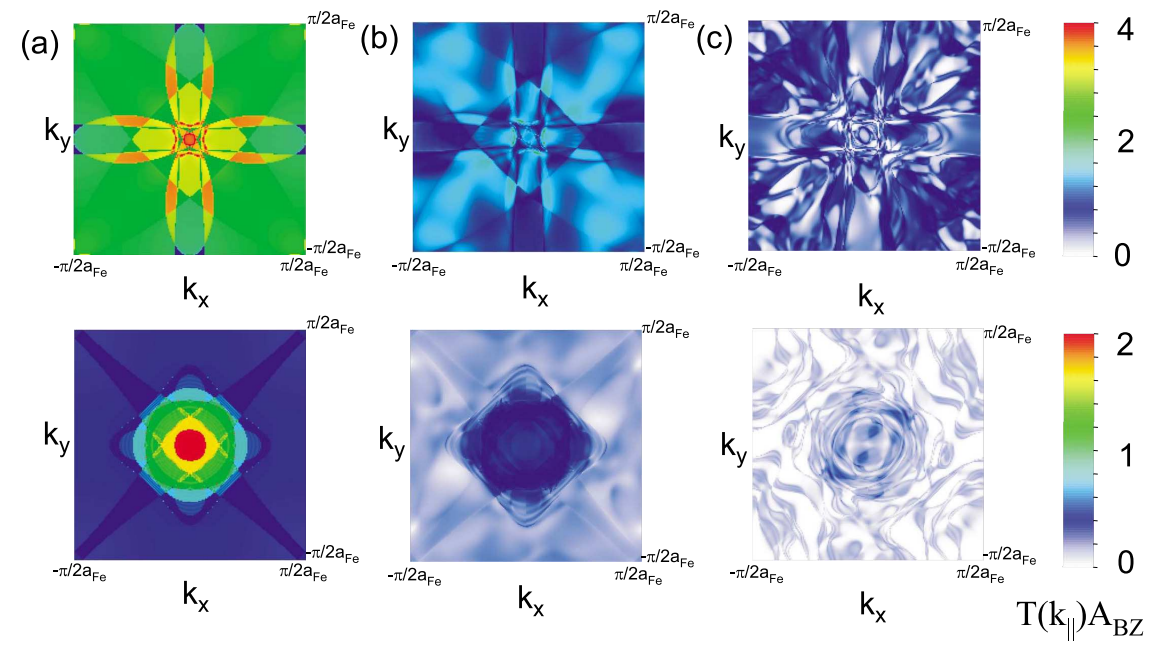

Abbildung 2.10: Karten spinabhängiger Leitfähigkeit $T^{\sigma}\left(k_{\|}\right)$von kristallinem Eisen (a), mit einer (b) und sieben (c) Lagen amorphen Eisens für den Minoritätsspinkanal (obere Reihe) und Majoritätsspinkanal (untere Reihe). Aus GRADHAnd ET AL. 41$]$

Die Ursache für die unerwartet hohen TMR-Werte in Systemen mit amorphen Elektroden war zunächst unklar. DJAYAPRAWIRA ET AL. vermuteten zunächst eine größere Bedeutung der Barriere und der Grenzflächenrauigkeit für den TMR, gleichzeitig schlossen sie aber auch nicht aus, dass einige Monolagen der Elektroden an der Grenzfläche zum MgO auskristallisiert seien, obwohl sie dies in ih- 
ren TEM Untersuchungen nicht beobachten konnten. Zu diesen Zeitpunkt lagen auch noch keine theoretischen Untersuchungen zu magnetischen Tunnelelementen mit amorphen Elektroden und kristallinen Barrieren vor, diese erfolgten 2007 durch HeILIGER, GRADHAND ET AL. 16/41. Sie führten ihre Berechnungen für Modellsysteme mit ferromagnetischen Elektroden begrenzter Ausdehnung durch, im Gegensatz zu früheren Untersuchungen. Als Ausgleich ergänzten sie ausgedehnte nichtmagnetische bcc-Cu Elektroden als Reservoir für $\Delta_{1}$-artige Zustände.

Abbildung 2.10 zeigt zunächst die Auswirkung des amorph modellierten Eisens auf die $k_{\|}$-abhängige Leitfähigkeit in beiden Spinkanälen des Eisens. Hierbei wurde ein System modelliert, bei welchem 1 bzw. 7 Lagen amorphen Eisens zwischen kristallinen Eisenelektroden eingefügt wurden, und hierfür die Transmission berechnet. Es wird deutlich, dass die ehemals hohe Spinpolarisation um $k_{\|}=0$ und die hot spots schnell zerstört wird. Im Zusammenspiel mit der Filterwirkung des $\mathrm{MgOs}$ ist für amorphe Elektroden also kein großer TMR zu erwarten. Die ermittelte Gesamtleitfähigkeit des amorphen Eisens stimmte gut mit experimentellen Werten überein, weshalb die Modellierung als ausreichend für die weiteren Untersuchungen angesehen wurde.

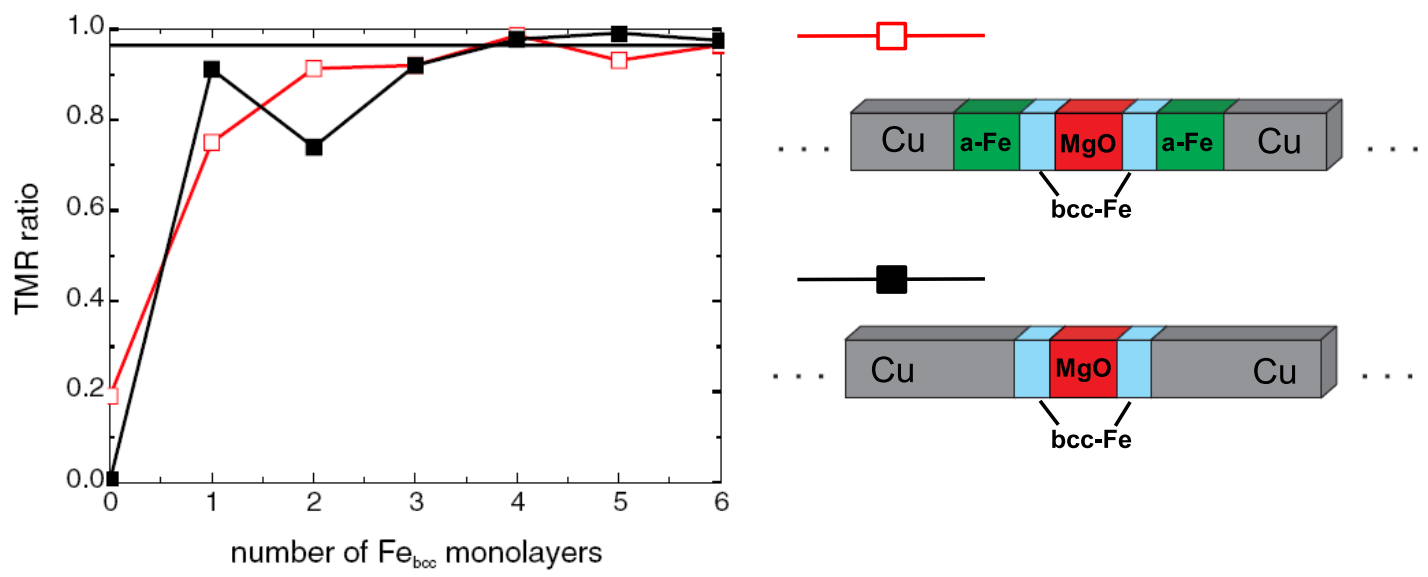

Abbildung 2.11: TMR-Werte in Abhängigkeit von der Anzahl kristalliner EisenMonolagen an der Grenzfläche zum MgO. Dargestellt ist der normierte TMR, der durch $\frac{G_{\mathrm{P}}-G_{\mathrm{AP}}}{G_{\mathrm{P}}+G_{\mathrm{AP}}}$ definiert ist. Die horizontale Linie entsprichte dem Wert für unendlich ausgedehnte kristalline Eisenelektroden, etwa 5000\% in optimistischer Definition. Aus HEILIGER ET AL. 16

In Abbildung 2.11 ist der TMR in Abhängigkeit der Anzahl kristalliner Monolagen Eisens für zwei unterschiedliche Systeme dargestellt, zum einen ein System 
mit ausschließlich kristallinen Monolagen Eisen an der Grenzfläche und zum anderen ein System bei welchem die kristallinen Eisenlagen um einen amorphen Teil ergänzt werden, dessen Dicke vier kristallinen Monolagen entspricht. Im Falle ohne amorphes Eisen gibt es für null Monolagen trivialer Weise keinen TMR, da das Tunnelsystem vollständig nichtmagnetisch ist. Bei rein amorphen Elektroden ohne kristallines Eisen an der Grenzfläche beträgt der TMR lediglich 44\%, was vergleichbar mit den Erwartungen nach dem Jullière Modell ist. Aber für nur zwei kristalline Monolagen zwischen der Barriere und dem amorphen Eisen steigt der TMR auf 560\% nach der optimistischen Rechnung. Mit mehr als sechs kristallinen Lagen Eisen verhalten sich die beiden Systeme nahezu identisch, so dass die amorphe Eisenschicht in diesem Falle keinen Einfluss mehr auf den TMR hat. Dies zeigt deutlich die große Bedeutung der Kristallinität der ferromagnetischen Elektroden in der Nähe der Grenzfläche zur Barriere für den TMR.

\subsubsection{Sauerstofffehlstellen}

Nachdem in den vorangegangenen Abschnitten vor allem auf die Störung der Spinpolarisation in den ferromagnetischen Elektroden eingegangen wurde, widmen wir uns nun der Störung der Symmetrieerhaltung in der kristallinen MgO Barriere. In den Messungen von MiAO ET AL. ${ }^{42}$ wurde eine starke Abhängigkeit des Tunnelmagnetowiderstands sowie der übrigen Transporteigenschaften, wie I(U)Abhängigkeit oder Flächenwiderstand, von den Präparationsbedingungen für die $\mathrm{MgO}-$ Barriere gefunden. Für eine Barriere, die mittels $\mathrm{MBE}$ bei $180^{\circ} \mathrm{C}$ gewachsen wurde, fanden sie die typischen Merkmale für kohärentes Tunneln und einen TMR von $130 \%$ bei Raumtemperatur. Eine Probe mit einer bei Raumtemperatur gewachsenen Barriere zeigte hingegen nur 42\% TMR, einen wesentlich höheren Flächenwiderstand und deutlich anderes I(U)-Verhalten (siehe Abbildung 2.12a).

Aus früheren Untersuchungen war bereits bekannt, dass $\mathrm{MgO}$ bei der MBE bevorzugt sauerstoffdefizitär wächst 12143 . Daher wurde vermutet, dass das Sauerstoffdefizit mit steigender Wachstumstemperatur abnimmt und dass Sauerstofffehlstellen in der Barriere, die als Streuzentren für die tunnelnden Elektronen dienen, die Ursache für die veränderten Transporteigenschaften sind. Hierfür wurde ein Modell entwickelt, dass zwei getrennte Kanäle für kohärentes und elastisches Tunneln vorsieht. Wie in Abbildung 2.12b ersichtlich, findet bis zum Streuzentrum kohärentes Tunneln statt und nach dem Verlust der Symmetrie elastisches. Die beiden Strombeiträge sind also zum einen alle nicht gestreuten, kohärent tunnelnden Zustände und zum anderen alle an Störstellen gestreuten Zustände. Insbesondere die Streuung aus den für hohen TMR hauptverantwortlichen $\Delta_{1^{-}}$ Zuständen führt zur deutlichen Abnahme des TMR. Wegen der geringen Abklingrate im $\mathrm{MgO}$ können die fehlenden $\Delta_{1}$-Zustände auch den Widerstandsanstieg 
a)

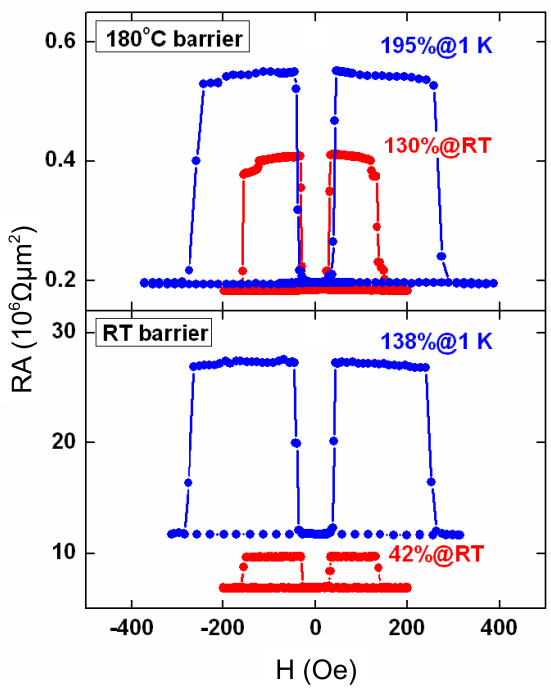

b)

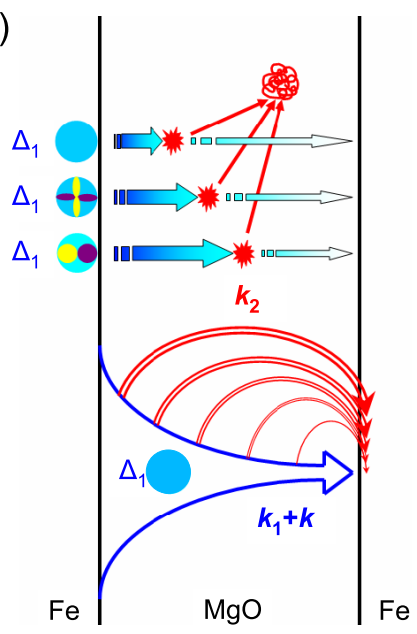

Abbildung 2.12: Vergleich der Magnetowiderstandskurven für verschiedene Wachstumstemperaturen der MgO-Barriere (a) und schematische Darstellung des nicht kohärenten Tunnelns (b). Aus Miao ET AL. 42

zum Teil erklären. Hierdurch wäre ein um das fünffache höherer Widerstand im parallelen Fall zu erwarten, dass die Widerstandsänderung noch deutlicher ausfällt führen die Autoren auf weitere Effekte der erhöhten Wachstumstemperatur, zum Beispiel erhöhte Filmrauigkeit, zurück. Unter der Annahme, dass die gemessenen Werte für die $180^{\circ} \mathrm{C}$ Barriere dem kohärenten Tunneln entsprechen und einer Abschätzung des TMR von 38\% für den elastischen Kanal nach dem Jullière Modell, lässt sich aus den gemessenen Daten für die Raumtemperaturbarriere eine charakteristische Streulänge von 1,6 $\mathrm{nm}$ bestimmen. 


\section{Experimentelle Methoden}

Dieses Kapitel beschreibt die eingesetzten experimentellen Methoden. Es gliedert sich dabei in zwei Teile. Im ersten Teil werden die Verfahren vorgestellt, die zur Herstellung der Proben und zur präparativen Vorbereitung für die folgenden Messungen verwendet wurden. Der zweite Teil befasst sich mit den benötigten Messmethoden und Analyseverfahren.

\subsection{Probenpräparation}

Die in dieser Arbeit untersuchten Proben stammen zum Teil aus verschiedenen Arbeitsgruppen, mit denen Kooperationen unterhalten werden. Allen Proben gemein ist die Vorgehensweise, dass das TMR-System zunächst als vollflächiger Schichtstapel auf einem geeigneten Substrat präpariert wird. Anschließend werden daraus mittels Photo- oder Elektronenstrahllithographie und einem nachfolgenden Ätzschritt die einzelnen TMR-Elemente herausgearbeitet. Die einzelnen Schichten des TMR-Systems werden dabei entweder durch Elektronenstrahlverdampfen oder mittels Magnetronsputtern hergestellt, deren Unterschiede im Folgenden erläutert werden. Nachdem die elektrischen Transporteigenschaften der TMRElemente vermessen wurden, können mit einem fokussierten Ionenstrahl gezielt Querschnitte aus einigen TMR-Elementen geschnitten werden für die strukturelle Untersuchung der Grenzflächen mit Hilfe der Transmissionselektronenmikroskopie.

\subsubsection{Elektronenstrahlverdampfen}

Das Elektronenstrahlverdampfen ist ein thermisches Aufdampfverfahren im Ultrahochvakuum (UHV), bei welchem das zu verdampfende Material von einem konzentrierten Elektronenstrahl lokal stark aufgeheizt wird, während es auf der Rückseite durch den wasserdurchflossenen Tiegel, in dem es liegt, gekühlt wird. Der hierdurch entstehende Temperaturgradient sorgt dafür, dass nur im Bereich des auftreffenden Elektronenstrahls eine hinreichende Temperatur erreicht wird, um das Material zu verdampfen, und eine punktförmige Quelle für Atome entsteht. Aufgrund diese Eigenschaft ist das Verfahren gut geeignet, um Proben ge- 
gebenenfalls durch einfache Schattenmasken oder ein lithographisches Verfahren mit einem Lift-Off-Schritt zu strukturieren. Elektronenstrahlverdampfen eignet sich für viele Materialien, insbesondere Metalle aber auch Isolatoren, die sich stöchiometrisch verdampfen lassen, wie beispielsweise $\mathrm{MgO}$. Weniger gut geeignet ist es zum direkten Verdampfen von Legierungen, da die Legierungsbestandteile in der Schmelze im Allgemeinen unterschiedliche Dampfdrücke haben und die Zusammensetzung der erzeugten Schicht so nicht der Zusammensetzung des Quellmaterials entspricht, welche sich obendrein mit der Zeit durch diese Entmischung auch noch verändert. Es besteht allerdings die Möglichkeit Legierungen durch Koverdampfen ihrer Bestandteile aus verschiedenen Tiegeln zu erzeugen. Der charakteristische Unterschied zum Sputtern (siehe unten) ist, dass die Atome ungehindert das Substrat erreichen und dort mit Energien, je nach Siedetemperatur des Materials, von einigen $100 \mathrm{meV}$ auftreffen.

Die in dieser Arbeit untersuchten elektronenstrahlverdampften Proben wurden in einer UHV-Anlage mit zwei Thermionics Four Position Crucible e-Gun Source 100-0040 Elektronenstrahlverdampfer, die jeweils vier Tiegel für Materialien bereitstellen, gefertigt.

\subsubsection{Magnetronsputterdeposition}

Beim Sputtern wird das Material nicht durch Verdampfen in die Gasphase gebracht sondern es wird durch Beschuss mit hochenergetischen Ionen und dem daraus folgenden Impulsübertrag von der Oberfläche her abgetragen. Ist dieser Materialabtrag das Ziel der Bemühungen, so spricht man von Sputterätzen, welches bei Strukturierungsprozessen eingesetzt wird. Wird hingegen die Wiederabscheidung des abgetragenen Materials auf einem Substrat in der Nähe genutzt, spricht man von Sputterdeposition.

Die nötigen hochenergetischen Ionen werden durch ein Plasma in einer verdünnten Gasatmosphäre, meist Edelgase wie Argon, erzeugt. Diese werden im einfachsten Fall, dem sogenannten DC-Sputtern, durch eine negative Bias-Spannung auf das abzutragende Material, das Target, beschleunigt. Dort übertragen sie ihre kinetische Energie in Stoßkaskaden an das Target-Material und schlagen so einzelne oder mehrere Atome aus diesem heraus. DC-Sputtern funktioniert gut mit allen elektrisch leitfähigen Target-Materialien. Isolierende Materialien würden sich aber schnell aufladen und die Ionen somit abbremsen, deshalb weicht man hierfür auf das RF (Radio Frequency)-Sputtern aus, bei welchem eine hochfrequente Wechselspannung an das Target-Material angelegt wird, um die Aufladung zu verhindern.

Bei der speziellen Form des Magnetronsputterns wird über dem Target-Material zusätzlich zum elektrischen Feld noch ein Magnetfeld erzeugt, welches die leichten 
Elektronen des Plasmas auf Spiralbahnen zwingt, die Ionen aber kaum beeinflusst. In Bereichen in denen die Magnetfeldlinien parallel zur Targetoberfläche verlaufen wird dadurch eine stark erhöhte Ionisation und damit unterhalb dieser Bereiche ein erhöhter Abtrag erreicht.

Im Gegensatz zum Elektronenstrahlverdampfen erreichen die Targetatome das Substrat beim Sputtern nicht ungehindert. Sie geben einen Teil ihrer zunächst relativ hohen kinetischen Energie durch Stöße an das Sputtergas ab. Die Restenergie mit der sie das Substrat erreichen, welche neben der Substrattemperatur großen Einfluss auf das Wachstumsverhalten des Films hat, ist also von vielen Faktoren wie Gasdruck, Geometrie und Beschleunigungsspannung abhängig und nicht vorwiegend ein Materialparameter wie beim Elektronenstrahlverdampfen. Da ferromagnetische Targets die magnetischen Feldlinien zum Teil kurzschließen und so die Bedingungen beim Magnetronsputtern verschlechtern, ist für sie ein relativ hoher Druck des Sputtergases erforderlich. Durch das großflächige Abtragen des Targetmaterials und die diffuse Ausbreitung der Atome durch das Sputtergas ist es möglich großflächig homogene Filme zu präparieren. Da auf diese Weise beim Sputtern kleinskalige Inhomogenitäten des Targets ausgeglichen werden können, werden für Legierungen häufig Targets verwendet, die pulvermetallurgisch durch Pressen und Sintern aus den pulverförmigen Legierungsbestandteilen hergestellt werden. Da außerdem keine Entmischung des Targets stattfindet, kann man eine über lange Zeit konstante Filmqualität erwarten.

\subsubsection{Strukturierung}

Nach der Präparation der vollflächigen TMR-Systeme müssen diese zunächst lateral strukturiert werden, um sie für die elektrischen Transportmessungen vorzubereiten. Dies geschieht in einem photolithographischen Negativprozess, in dem die Teile der Oberfläche, die als TMR-Elemente herausgearbeitet werden sollen, zunächst mit Photolack abgedeckt werden. Anschließend wird die unbedeckte Fläche durch Sputterätzen bis zur unteren Elektrode heruntergeätzt. Um dabei saubere Flanken zu erhalten und Redeposition an diesen zu vermeiden wird die Probe während des Ätzens rotiert und die Einfallsrichtung der Ionen um $27^{\circ}$ gegen die Probennormale verkippt. Werden dabei große Elemente mit mehreren $\mu \mathrm{m}$ Durchmesser erzeugt, ist die Strukturierung nach der Entfernung des restlichen Photolacks beendet, da diese Elemente direkt mit einer Probestation (vergl. Abschnitt 3.2.5 kontaktiert werden können. Falls aber kleine Elemente mit Durchmessern unter einem $\mu \mathrm{m}$ präpariert werden, ist es nötig zunächst die freiliegenden unteren Kontaktflächen mit einem geeigneten Isolator abzudecken und nach der Entfernung des restlichen Photolacks in einem weiteren Lithographieschritt obere Kontaktflächen aufzubringen. Eine detailierte Beschreibung der Strukturierung 
findet sich in der Diplomarbeit von KAI UBBEN ${ }^{44}$.

\subsubsection{Zielpräparation mittels FIB}

Mit Hilfe des Focussed Ion Beam (FIB) ist es möglich Proben sehr effektiv im Mikro- und Nanometerbereich zu strukturieren. Dazu wird ein stark fokussierter $\mathrm{Ga}^{+}$-Ionenstrahl mit Beschleunigungsspannungen zwischen $1 \mathrm{kV}$ und $30 \mathrm{kV}$ genutzt, um gezielt Material abzutragen oder abzuscheiden. Unter den vielfältigen Möglichkeiten, die dieses Gerät bietet, ist die Möglichkeit dünne Scheiben im Querschnitt aus geschichteten Probensystemen zu schneiden und diese anschließend soweit zu dünnen, dass sie für die Untersuchungen im Transmissionselektronenmikroskop (TEM) (Abschnitt 3.2.1) geeignet sind. Dabei ist es möglich, die Position, an welcher der Querschnitt ausgeführt wird, so genau zu wählen, dass dabei beispielsweise ein bereits auf einige $100 \mathrm{~nm}$ Durchmesser präpariertes und kontaktiertes TMR-Element getroffen wird.
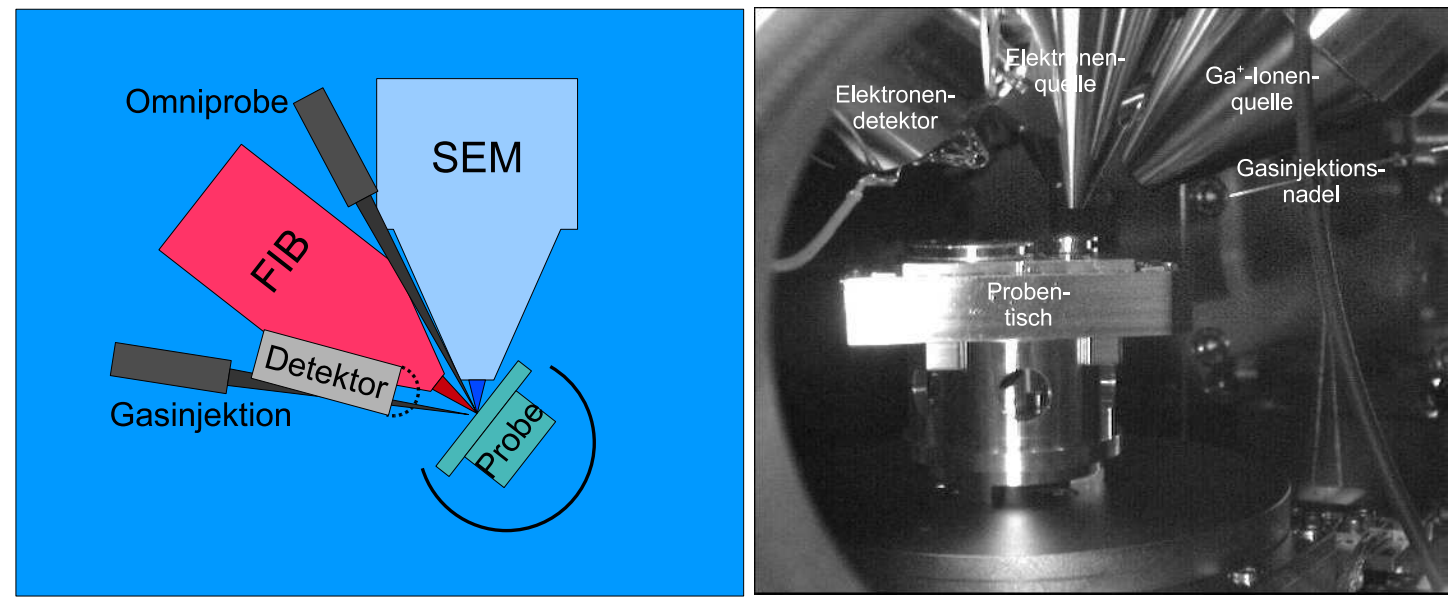

Abbildung 3.1: Schemazeichnung eines FIB (links) und Innenansicht des verwendeten FEI Nova 600 NanoLab (rechts).

Das für diese Arbeit verwendete FIB ist ein Zweistrahlgerät der Firma Fei vom Typ Nova 600 NanoLab (Abbildung 3.1, rechts). Neben dem fokussierten $\mathrm{Ga}^{+}$-Ionenstrahl enthält es ein Rasterelektronenmikroskop (SEM) mit welchem es auch möglich ist in situ den Arbeitsfortschritt des Ionenstrahls zu überprüfen. Des Weiteren verfügt das Gerät über ein Gasinjektionssystem zur chemischen Gasphasenabscheidung sowie über einen Mikromanipulator zum Transport kleiner Probenstückchen. Bei der chemischen Gasphasenabscheidung wird durch eine 
feine Nadel in wenigen zehntel Millimetern Abstand von der Probe ein metallorganischer Precursor eingeleitet, welcher wahlweise mittels Elektronenstrahl (e-beam assisted) oder Ionenstrahl (ion-beam assisted) chemisch aufgespalten wird. Das Metall, oder bei gleichzeitiger Oxidation auch ein Isolator, scheidet sich dabei lokal auf der Probe ab, und die organischen Bestandteile des Precursors werden durch das Vakuumsystem abgesaugt.
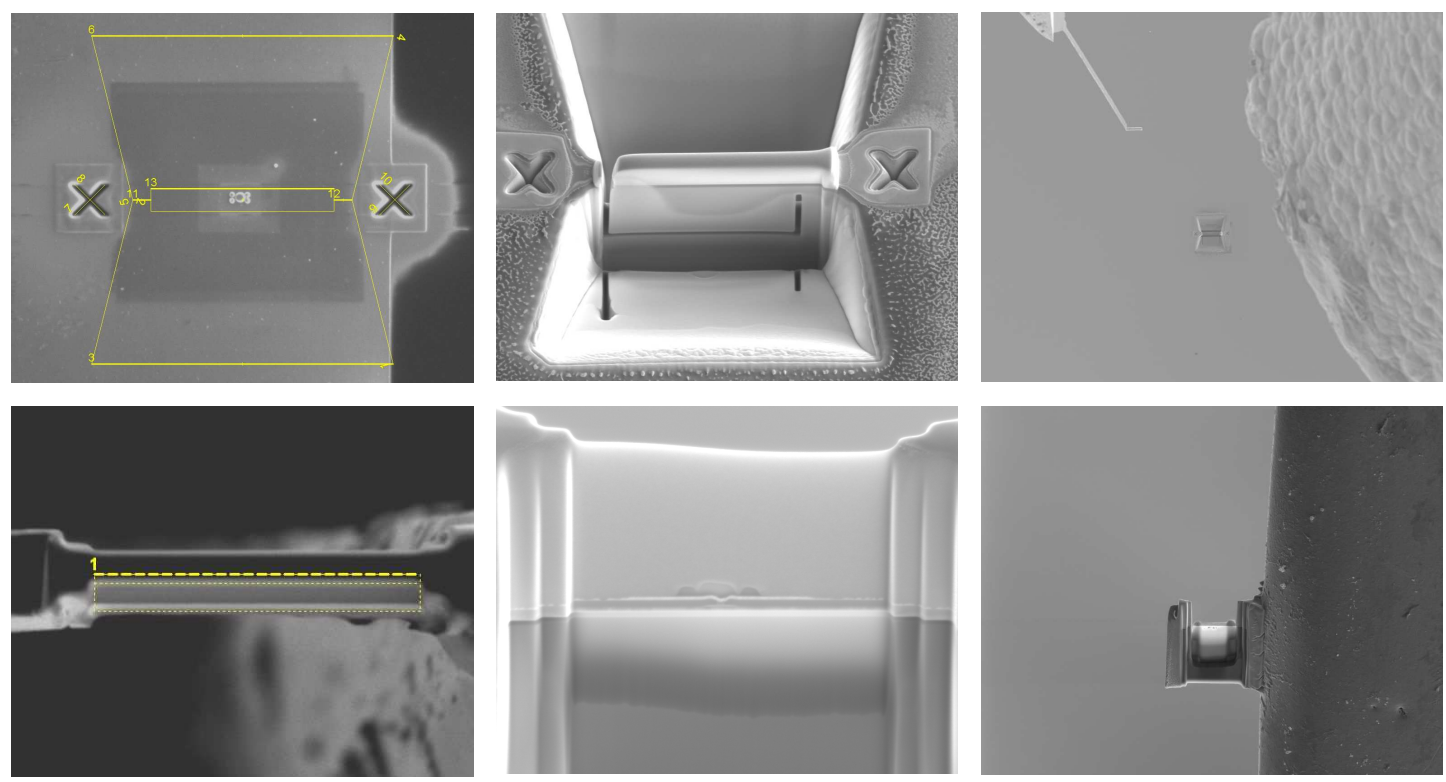

Abbildung 3.2: Arbeitsschritte bei der Zielpräparation einer TEM-Lamelle. Platindeposition und Marker für den AUTO-TEM Schritt (a), freigeschnittene Lamelle (b), Transport der Lamelle mit dem Mikromanipulator; rechts im Bild ist die Gasinjektionsnadel (c), Dünnungsschritt im Ionenbild (d), vorletzter Dünnungsschritt im Elektronenbild (e), fertige TEM-Lamelle am Kupfer-Grid (f).

Im Folgenden wird die Präparation einer TEM-Lamelle mit der sogenannten lift-out-Technik im Detail beschrieben.

Im ersten Schritt wird die Region von Interesse im Elektronenbild ausfindig gemacht und zunächst durch Abscheidung einer 0,5 um dicken Platinschicht vor späteren Beschädigungen durch den Ionenstrahl geschützt. Danach wird in einem automatischen Prozess (Auto-TEM) von zwei Seiten ein stufenförmiger Graben - zur späteren Lamelle hin tiefer werdend - mit großem Ionenstrom erzeugt, gefolgt von einem ersten, ebenfalls automatischen Dünnungsschritt mit abnehmen- 
dem Ionenstrom. Nun wird die Probe um $45^{\circ}$ zur Einfallsrichtung des Ionenstrahls verkippt und die Lamelle mit einem U-förmigen Schnitt soweit herausgetrennt, bis sie nur noch an einer Seite durch einen schmalen Steg mit dem Rest der Probe verbunden ist. Nachdem die Probenoberfläche wieder senkrecht zur Einfallsrichtung des Elektronenstrahls gestellt wurde, wird der Mikromanipulator an die Lamelle angenähert und dessen Spitze durch Platindeposition mit der Lamelle verbunden. Jetzt kann der verbliebene Steg mit dem Ionenstrahl aufgeschnitten und die Lamelle endgültig von der Probe getrennt werden. Sie wird nun vorsichtig aus der Probe herausgezogen und mit dem Mikromanipulator an ein kohlenstoffbeschichtetes kupfernes TEM-Grid bis zum Kontakt angenähert. Nach dem Verschweißen der Lamelle mit dem TEM-Grid durch Platindeposition von beiden Seiten, kann der Mikromanipulator wieder durch einen Schnitt mit dem Ionanstrahl von der Lamelle gelöst werden. Erst jetzt erfolgen die endgültigen Dünnungsschritte mit dem Ionenstrahl bei $30 \mathrm{kV}$ abwechselnd von beiden Seiten mit abnehmenden Ionenstrom von 300 bis 30 pA unter ebenfalls abnehmenden Einfallswinkeln von $1,2^{\circ}-0,5^{\circ}$. Zum Schluss werden beide Seiten der Lamelle unter einem Einfallswinkel von $7^{\circ}$ bei einem Strom von $30 \mathrm{pA}$ und einer Beschleunigungsspannung von $5 \mathrm{kV}$,abgeduscht“. Dies soll zumindest teilweise die durch den Ionenstrahl amorphisierte Oberfläche sowie implantierte Galliumatome wieder entfernen.

\subsection{Untersuchungsmethoden}

Im Folgenden Abschnitt werden die für diese Arbeit relevanten Untersuchungsmethoden vorgestellt. Zunächst werden die Grundlagen der Transmissionselektronenmikroskopie eingeführt. Der anschließende Teil befasst sich mit der Mittelung elektronenmikroskopischer Abbildungen. Danach geht es darum, wie solche gemittelte Abbildungen simuliert werden können und wie man ein geeignetes Modellsystem als Ausgangspunkt für die Simulationen generiert. Insbesondere wird eine Abwandlung des Modells von BorgARDT ET AL. ${ }^{45}$ betrachtet, welche es an das hier vorliegende System anpasst. Zuletzt folgt ein kurzer Abschnitt über elektrische Transportmessungen.

\subsubsection{Transmissionselektronenmikroskopie}

Das Transmissionselektronenmikroskop ist ein wirkungsvolles Instrument für die Aufklärung der mikrostruktureller Eigenschaften von Materialien und Bauelementen. Hierzu wird ein Elektronenstrahl von einer Kondensoroptik auf die zu untersuchende Probe abgebildet, welche für die Elektronen transparent sein muss. Nach dem Durchgang durch die Probe werden die Elektronen von einem Linsen- 
system über mehrere Zwischenbilder auf einen Schirm bzw. eine CCD-Kamera abgebildet. Dabei kann mit der Zwischenlinse ausgewählt werden, ob das TEM im mikroskopischen Abbildungsmodus (Abbildung $3.3 \mathrm{a}$ ) oder im Beugungsmodus (Abbildung 3.3b) arbeitet, je nachdem, ob eine Realraumabbildung oder ein Beugungsmuster auf dem Schirm abgebildet werden soll. Beim Durchgang des Elektronenstrahls durch die Probe kommte es zu verschiedenen Streuprozessen. Dabei ist das Streupotential zwar materialabhängig, aber als Richtwert lässt sich sagen, dass die Proben, um die Eletronentranzparenz zu gewährleisten, bei geringerer Auflösung höchstens einige $100 \mathrm{~nm}$ und für atomare Auflösung sogar nur etwa $10 \mathrm{~nm}$ dick sein dürfen.

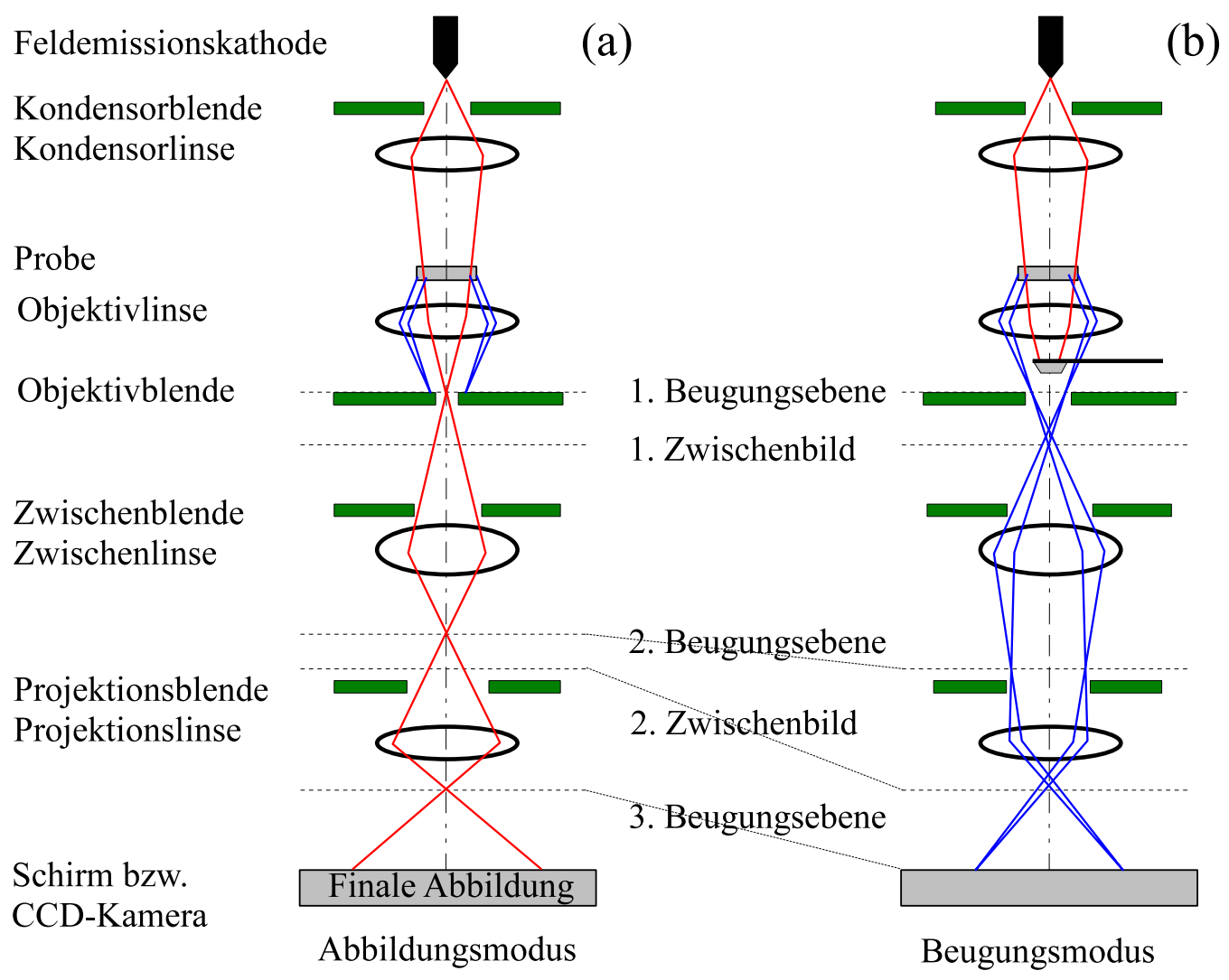

Abbildung 3.3: Anordnung der verschiedenen Komponenten eines TEM mit je einem Strahlengang für den Abbildungsmodus (a), um Informationen im Realraum zu gewinnen, und für den Beugungsmodus (b), um Informationen im reziproken Raum zu gewinnen. 
Zur Kontrastentstehung einer TEM-Abbildung können verschiedene Wechselwirkungen zwischen Elektronen und Probe beitragen. So werden Elektronen beim Durchgang durch die Probe elastisch wie inelastisch gestreut. Die Stärke dieser Streuung ist abhängig von der Kernladungszahl und Anzahl der passierten Atome. Für eine Hellfeldabbildung werden mit der Objektivblende nun in der Objektivbrennebene die stärker gestreuten Elektronen aus dem Strahlengang entfernt, was dazu führt, dass die strärker streuenden Bereiche der Probe in der elektronenmikroskopischen Abbildung dunkel erscheinen, die weniger stark streuenden hell (Hellfeldabbildung). Durch die Öffnung der Objektivblende kann dieser Streuabsorptionskontrast oder auch Massendickekontrast eingestellt werden.

Wird die Objektivblende verschoben, so dass nur ein Streuwinkelbereich selektiert wird und alle weniggestreuten Elektronen ausgeblendet werden, entsteht eine Dunkelfeldabbildung. Hierdurch können bestimmte Details der Probe besser hervorgehoben werden.

Bei kristallinen Proben kommt es, neben der diffusen Streuung, auch zu BraggStreuung durch die Netzebenen des Kristalls. Diese führt zu einer starken Abhängigkeit des Kontrastes von der Kristallorientierung relativ zum Elektronenstrahl. Aufgrund der Orientierungsabhängigkeit kann dieser Beugungskontrast durch Verkippung der Probe vom Streuabsorptionskontrast separiert werden.

Insbesondere für die hochaufgelöste Transmissionselektronenmikroskopie (HRTEM) spielt eine weitere Form der Kontrastentstehung eine wesentliche Rolle: Die Elektronenwelle erfährt beim Durchgang durch die Probe aufgrund des Objektpotentials einen Phasenschub. Durch die abbildenende Optik entsteht hieraus durch Interferenz der sogenannte Phasenkontrast. Hierbei beeinflussen neben der Probenstruktur noch viele andere Eigenschaften des Elektronenstrahls sowie der Elektronenoptik die Art des Kontrastes. So tritt zum Beispiel auch in der Objektivlinse eine Phasenverschiebung zwischen achsennahen und -fernen Strahlen auf, die von den Aberrationen - insbesondere Öffnungsfehler und Abweichung vom Gaußschen Fokus (sog. Defokus) - abhängt. Durch die Objektivstromeinstellung (Defokussierung) kann der Phasenkontrast also beeinflusst werden. Eine Interpretation ist schwierig und wird meist erst möglich, indem die HRTEM-Abbildungen mit Simulationen verglichen werden, welche die Phasenverschiebungen der Elektronenwelle beim Durchgang durch die Probe und die anschließende abbildende Optik berechnen und die Kontrastentstehung durch Interferenz ermitteln. Mehr zur Simulation von HRTEM-Abbildungen findet sich in Kapitel 3.2.4.

Ein weiterer wichtiger Betriebsmodus für analytische Untersuchungen ist der scanning transmission electron microscope (STEM) Modus. Hierbei wird der Elektronenstrahl sehr fein auf die Probenebene fokussiert, wodurch ein sehr kleines lokales Anregungsgebiet erzeugt wird. Durch Rastern des Strahls bei gleichzeitiger Detektion gestreuter oder ungestreuter Elektronen können so Dunkel- und Hell- 
feldabbildungen im STEM-Modus aufgenommen werden. Ferner erlaubt dieser Modus die Aufzeichnung ortsaufgelöster Informationen über die Elementverteilung, beispielsweise durch energiedispersive Aufzeichnung der enstehenden Röntgenstrahlung (Röntgenspektrometrie, EDX).

\subsubsection{Dreidimensionale tomographische Rekonstruktion aus STEM-Abbildungen}

Selbst eine dünne durchstrahlbare TEM-Lamelle hat eine räumliche Tiefe, die deutlich größer sein kann als die laterale Auflösung der TEM-Abbildung, die immer nur einzweidimensionales Bild der dreidimensionalen Probe liefert. Mithilfe einer tomographischen Rekonstruktion können aber auch räumliche Informationen über die Probe aus einer Serie von TEM-Abbildungen zurückgewonnen werden.
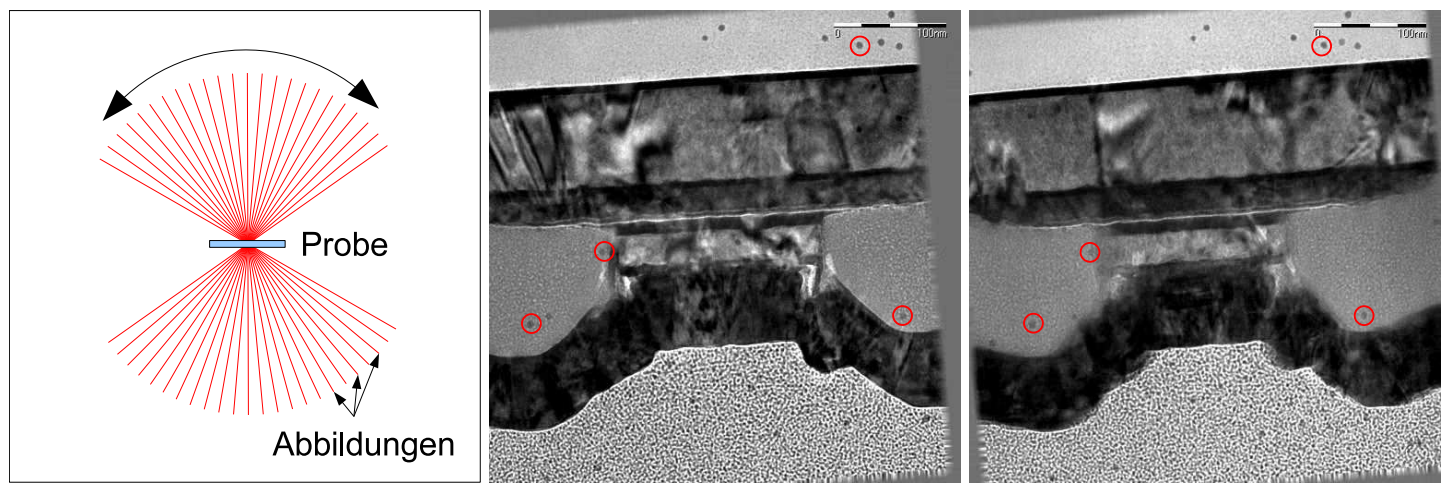

Abbildung 3.4: Schematische Darstellung der Datenaufnahme für tomographische Rekonstruktionen (links) und zwei TEM-Aufnahmen unter verschiedenen Einstrahlwinkeln. Einige der Goldmarker sind rot markiert.

Abbildung 3.4 illustriert das Verfahren der Datengewinnung für solch eine tomographische Rekonstruktion. Hierzu wird eine Serie von Aufnahmen für unterschiedliche Durchstrahlrichtungen über einen möglichst großen Winkelbereich aufgenommen. Für die anschließende Rekonstruktion wird die Probe vorher mit geeigneten Markern, zum Beispiel Gold-Nanopartikel, versehen. In Abbildung 3.4 sind zwei Aufnahmen aus einer solchen Reihe gezeigt, in denen auch die Marker zu erkennen sind. Anhand dieser Marker können die Abbildungen später aneinander ausgerichtet werden, um zum Beispiel Probendrift auszugleichen. Aus den ausgerichteten Abbildungen kann dann mittels Software ein dreidimensionales Tomogramm der Probe berechnet werden. 


\subsubsection{Mittelung von HRTEM Abbildungen}

Zur quantitativen Auswertung von HRTEM Abbildungen ist es notwendig diese mit simulierten Abbildungen zu vergleichen.

Bei der Untersuchung einer Grenzfläche zwischen kristallinen und amorphen Bereichen der Probe ist ein solcher direkter Vergleich aber nicht ohne Weiteres durchzuführen, da es, selbst wenn die Eigenschaften der Atomverteilung in der amorphen Lage bekannt sind, noch eine große Anzahl an konkreten Umsetzungen für dieselbe Nahordnung gibt. Daher wurde von BORGARDT ET AL. 17/45/46 und PLIKAT ${ }^{47 \mid 48}$ eine periodische Mittelung entlang der Grenzfläche vorgeschlagen. Diese Mittelung erlaubt es, eine Intensitätsverteilung zu erhalten, welche von der mittleren Verteilung des projizierten Potentials (Vergl. 3.2.4) der Atome in der amorphen Lage erzeugt wird.

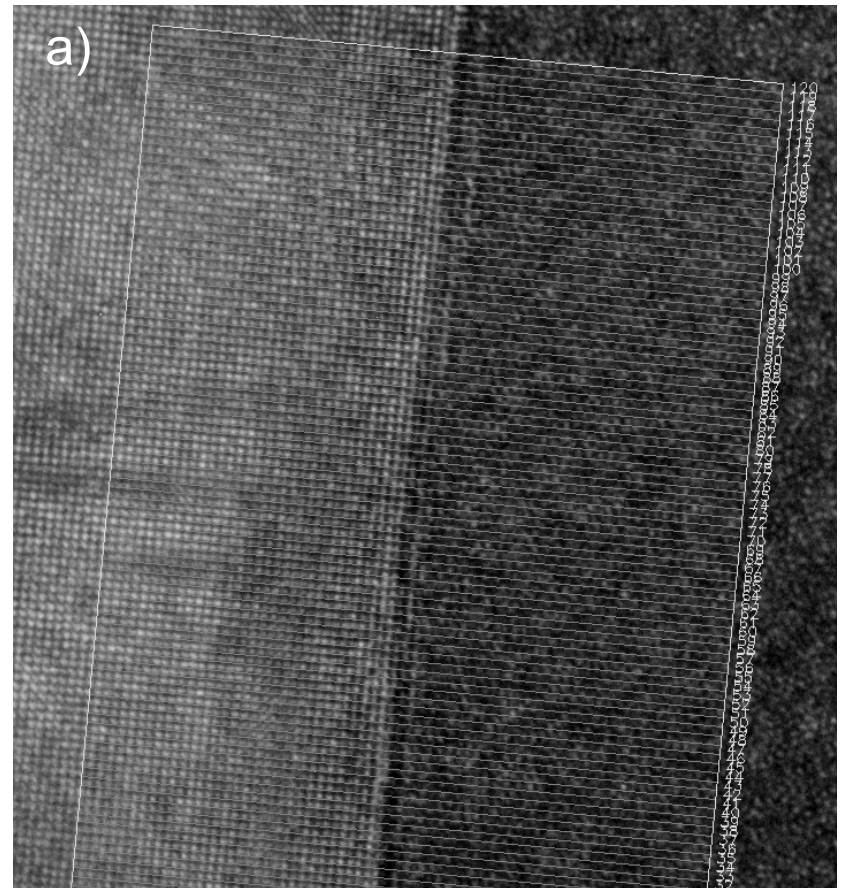

b)

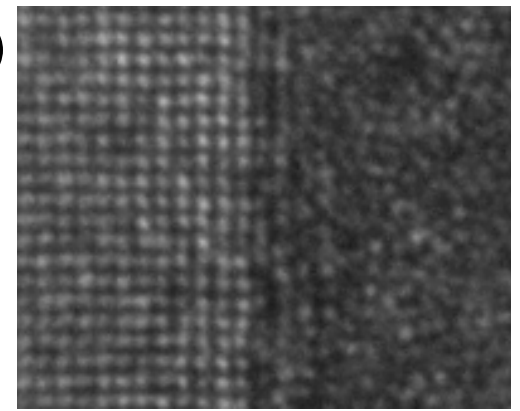

c)

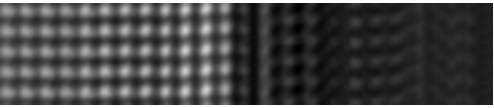

c)

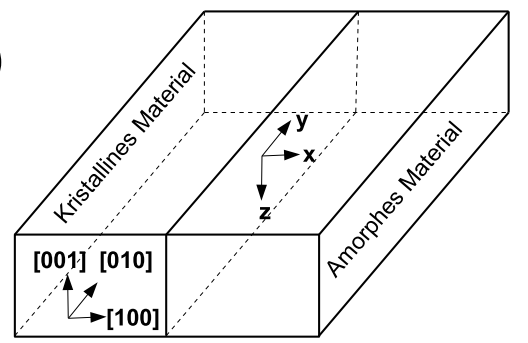

Abbildung 3.5: Vorgehen bei der Mittelung. Die HRTEM-Abbildung wird in Streifen senkrecht zur Barriere aufgeteilt (a). Durch Mittellung über eine bestimmte Anzahl an Streifen (b) erhält man gemittelte Abbildungen (c). Schematische Darstellung (d) zur Orientierung der Probe mit kriatallinen $\mathrm{MgO}$ auf der linken und amorphen $\mathrm{CoFeB}$ auf der rechten Seite. 
Die Vorgehensweise dabei ist in Abbildung 3.5 illustriert. Die HRTEM Abbildung wird zunächst in Streifen senkrecht zur Grenzfläche eingeteilt, deren Breite $d$ gerade der Periodizität im kristallinen Teil der Probe entspricht. Im Falle von $\mathrm{MgO}$, welches so ausgerichtet wird, dass sowohl die Grenzfläche als auch Durchstrahlrichtung $z$ parallel zu den Kubuskanten der Einheitszelle liegen, entspricht die Streifenbreite einer halben Gitterkonstante: $d=1 / 2 \cdot a_{\mathrm{MgO}}=0,21 \mathrm{~nm}$. Wegen der Stapelreihenfolge AB AB des MgO in z-Richtung sind benachbarte Streifen trotz unterschiedlicher Oberflächenatome in der Projektion nicht zu unterscheiden (vergl. Abbildungen 2.4 und 2.8). Anschließend wird eine Mittelung der Intensität über äquivalente Punkte in allen Streifen durchgeführt, wodurch es möglich wird, die kristallperiodisch korrelierten Anteile aus den Intensitätsverteilungen zu extrahieren:

$$
I^{\prime}(x, y)=\frac{s_{d}(y)}{N} \sum_{n=-(N-1) / 2}^{(N-1) / 2} I(x, y+n d),
$$

dabei ist $I^{\prime}(x, y)$ die gemittelte Intensitätsverteilung, $I(x, y)$ die Intensitätsverteilung in den Streifen der experimentellen Abbildung, $s_{d}$ die Spaltfunktion, welche den Wert eins annimmt für $-d / 2 \leq y<d / 2$ und null sonst. $N$ repräsentiert die Anzahl der Streifen.

Durch dieses Vorgehen wird die Information aus der Grenzflächenabbildung auf die $d$-periodischen Anteile reduziert. Abbildung 3.5. zeigt das Ergebnis einer gemittelten Grenzflächenabbildung, wobei die Mittelung lediglich einen Streifen liefert, welcher hier zur besseren Veranschaulichung wiederholt untereinander dargestellt ist. Im linken Teil der mittleren Grenzflächenabbildung reproduziert sich, wie zu erwarten, das kristalline Muster des MgO. Auf der rechten Seite ergibt sich mit zunehmendem Abstand von der Grenzfläche eine immer homogenere Intensitätsverteilung, welche durch die zufällige Verteilung der Atome im amorphen Teil hervorgerufen wird.

\subsubsection{Multislice-Simulation gemittelter Abbildungen}

Bei der Simulation elektronenmikroskopischer Abbildungen wird zunächst die Veränderung der einfallenden Elektronenwelle durch Streuung an der Probe berechnet. In einem weiteren Schritt werden dann die Abbildungseigenschaften des Mikroskops (Transferfunktion) berücksichtigt. Eine Möglichkeit, die Streuung der Elektronenwelle an der Probe zu berechnen, ist ein Blochwellenansatz, dessen Genauigkeit mit der Anzahl der berücksichtigten Beugungsreflexe ansteigt. Mit diesem Ansatz lassen sich allerdings ausschließlich einkristalline Objekte berechnen; sobald Defekte oder gar amorphe Bereiche berücksichtigt werden sollen ist er 
ungeeignet. Für solche Fälle hat sich die Multislice-Methode ${ }^{49-52}$ als etabliertes Verfahren zur Simulation von TEM Abbildungen herausgebildet.

Die Multislice-Methode ist zum Beispiel im EMS-Programmpaket von STADELMANN ${ }^{53}$ integriert. Hierbei wird das Modell der Probe, welches die Atompositionen enthält, in mehrere dünne Schichten (slices) senkrecht zur Elektronenstrahlrichtung unterteilt. Die gesamte Modifikation der Elektronenwelle wird durch die wiederholte Streuung am projizierten Potential dieser dünnen Schichten simuliert. Der Streuprozess an jeder Scheibe $j$ ist dabei in zwei Einzelschritte aufgeteilt:

1. Transmission: Statt die Streuung der einfallenden Elektronenwelle am 3-dimensionalen Potential der Atomverteilung zu berechnen, wird das Potential zunächst auf die 2-dimensionale Eintrittsfläche projiziert. Die Streuung an diesem Potential $\varphi^{(j)}(x, y)$ wird durch die Transferfunktion $P^{(j)}(x, y)$ beschrieben, durch welche der Elektronenwelle eine Phasenverschiebung proportional zum projizierten Potential aufgeprägt wird. Dies ist möglich, solange für jede Schicht die Phasennäherung erfüllt ist, welche fordert, dass die einfallende Elektronenwelle beim Durchlaufen der Probenscheibe relativ zur Vakuumausbreitung über dieselbe Strecke lediglich einen Phasenschub erfährt.

$$
\begin{aligned}
P^{(j)}(x, y) & =\exp \left(i \sigma \varphi^{(j)}(x, y)\right) \\
\operatorname{mit} \sigma & =\frac{\pi}{\lambda U_{R}}
\end{aligned}
$$

Dabei bezeichnet $\sigma$ die Wechselwirkungskonstante, welche durch die Wellenlänge $\lambda$ der Elektronen und die relativistisch korrigierte Beschleunigungsspannung des Mikroskops $U_{R}$ bestimmt ist.

Auf den Index $j$ kann im Folgenden verzichtet werden, da in dieser Arbeit nur Fälle betrachtet werden, deren Streupotentiale für alle Streifen identisch sind.

2. Propagation: Nach der Streuung der Elektronenwellen am projizierten Potential der $j$-ten Schicht breitet sie sich bis zur Eintrittsfläche der $j+1$-ten Schicht ungehindert im Vakuum über die Strecke $\Delta z$ aus. Diese Ausbreitung wird durch Faltung mit dem Fresnelpropagator

$$
\operatorname{FP}_{\Delta z}(x, y)=\frac{1}{i \lambda \Delta z} \exp \left[\frac{i \pi}{\lambda \Delta z}\left(x^{2}+y^{2}\right)\right]
$$

beschrieben. 


\section{Die "Averaged-Projected-Potential"-Näherung}

Es gibt zwei Wege gemittelte Abbildungen, wie sie in Abschnitt 3.2 .3 berechnet wurden, zu simulieren.

Beim atomistischen Ansatz werden die mittels der Multislice-Methode simulierten Abbildungen im nachhinein gemittelt, wie dies auch für die experimentellen Daten in Abschnitt 3.2.3 vorgestellt wurde. Hierbei gehen in die Simulationen konkrete Atompositionen ein, welche für die kristalline Seite auch bekannt sind, aber für den amorphen Bereich erst, beispielsweise mittels Molekulardynamiksimulationen, berechnet werden müssen. Wegen der hohen Anzahl an möglichen Realisierungen einer amorphen Verteilung durch diskrete Atompositionen ist dieser Ansatz nicht besonders sinnvoll. Hinzu kommt die Tatsache, dass Systemgrößen von $10^{4}-10^{5}$ Atomen gerechnet werden müssten, um mit den experimentell zugänglichen Mittelungsbereichen vergleichbare Ergebnisse zu erreichen. Dies würde zu unnötig langen Rechenzeiten führen oder enorme Rechenleistung erfordern.

Eine elegantere Alternative bietet die direkte Simulation gemittelter Abbildungen mittels der von BORGARDT ${ }^{17}$ vorgeschlagenen "Averaged-ProjectedPotential" (APP)-Näherung. Im Gegensatz zum atomistischen Ansatz wird hierbei das projizierte Potential $\varphi(x, y)$ durch ein mittleres projiziertes Potential $\varphi^{\mathrm{APP}}(x, y)$ für den amorphen Bereich ersetzt. Anstatt aus diskreten Atompositionen wird dieses aus einer mittleren 2-dimensionalen atomaren Dichteverteilung $\rho(x, y)$ innerhalb eines Streifens der Breite $d$ erzeugt. Die Breite $d$ entspricht dabei wieder der Periode in der kristallinen Abbildung, vergleichbar mit der Streifenbreite in Abschnitt 3.2.3. Dieses Verfahren spart gegenüber dem atomistischen Ansatz sehr viel Rechenzeit. Eine genauere Erläuterung der APP-Näherung findet sich bei THIEL ${ }^{18}$.

\section{Konstruktion einer zweidimensionalen Verteilungsfunktion}

Für die Konstruktion der zweidimensionalen Verteilungsfunktion $\rho(x, y)$ des amorphen Materials als Ausgangspunkt für die Simulation wird für kovalent gebundene Materialien ein rekursives Verfahren angewendet. Dabei wird für den kristallinen Teil der Probe die Näherung eines starren Substrats angenommen, d.h. dass die Positionen der Atome der letzten kristallinen Lage nicht durch das amorphe Material beeinflusst werden. Eine solche Näherung wird auch bei der elektronenmikroskopischen Untersuchung von fest/flüssig Grenzflächen für den festen Teil der Probe angenommen [54.

Von dieser Annahme ausgehend wird bei Borgardt ET AL. ${ }^{45}$ und ThIEL ${ }^{18}$ eine Konstruktionsvorschrift angewendet, welche die kristalline Struktur des Substrats Atomlage für Atomlage in das amorphe Material hinein fortsetzt. Dabei 


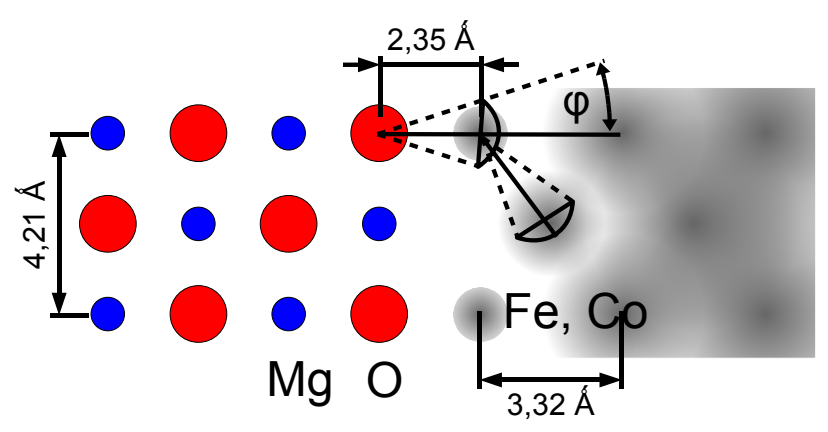

Abbildung 3.6: Konstruktion einer mittleren atomaren Verteilungsfunktion

wird sowohl für die Variation der Bindungslänge als auch für die Bindungswinkelverteilung um die mittlere Orientierung je eine Gaußverteilung benutzt. Die rekursive Fortsetzung dieser Vorgehensweise führt zu einem allmählichen Verschwinden der kristallinen Ordnung und einem Übergang zu einer homogenen Verteilung mit zunehmendem Abstand von der Grenzfläche.

Das in dieser Arbeit untersuchte System mit MgO/Fe-Grenzflächen unterscheidet sich in zweierlei Hinsicht von den a-Ge/c-Si bzw. a-Si/c-Si Grenzflächen, die von THIEL ${ }^{18}$ untersucht wurden. Zum einen haben $\mathrm{MgO}$ und Fe unterschiedliche Kristallstrukturen, welche aber pseudomorph aufeinander wachsen können, wie in Abschnitt 2.2.2 dargestellt. Zum anderen hat das amorphe Material CoFeB eher einen metallischen Bindungscharakter im Gegensatz zu den kovalenten Bindungen in Germanium oder Silizium. Aus diesem Grund wurde das Verfahren dahingehend abgewandelt, dass auf die Unterscheidung von Bindungswinkelvariation und Bindungslängenvariation verzichtet wird. Stattdessen wird eine isotrope gaußförmige Verteilung um die Positionen in einer pseudomorph kristallin aufwachsenden Schicht angenommen. Die Breite dieser Gaußverteilungen $\sigma_{i}$ wird dabei aus einer Bindungswinkelvariation $\varphi$ als einzigem Parameter und dem Atomabstand $a_{i}$ zum jeweils nächsten Atom der vorherigen Lage ermittelt (siehe Abbildung 3.6). Für die erste amorphe Lage ist dies der Abstand $a_{1}=a_{\mathrm{O}-\mathrm{Fe}}=2,35 \AA$ und damit gleich dem Abstand zwischen der letzten kristallinen Lage MgO und der ersten Lage Eisen entnommen aus der Veröffentlichung von MEYEHEIM ET AL. ${ }^{55}$. Damit ergibt sich für die erste amorphe Lage eine Gaußverteilung für die Wahrscheinlichkeit ein Eisenatom $\mathrm{zu}$ finden mit der Breite $\sigma_{\mathrm{O}-\mathrm{Fe}}=a_{\mathrm{O}-\mathrm{Fe}} \cdot \tan \varphi$ um die Position, 
welche es im kristallinen Eisen hätte:

$$
W=\frac{1}{\sqrt{2 \pi} \sigma_{\mathrm{O}-\mathrm{Fe}}} \exp \left(-\frac{1}{2} \frac{r^{2}}{\sigma_{\mathrm{O}-\mathrm{Fe}}^{2}}\right)
$$

mit der Abweichung $r$ zur Position eines Atoms im kristallinen Fall. Innerhalb des amorphen Materials entspricht der für die Breite der Verteilung maßgebliche Atomabstand dem Abstand nächster Nachbarn im kubisch-raumzentrierten Gitter des Eisens $a_{\mathrm{Fe}-\mathrm{Fe}}$ und damit ist die Breite der Gaußverteilung relativ zur vorherigen Lage $\sigma_{\mathrm{Fe}-\mathrm{Fe}}$. Dabei wurde zunächst eine volumenerhaltende tetragonale Verzerrung des Eisengitters angenommen, um die Gitterfehlpassung zwischen Eisen und $\mathrm{MgO}$ auszugleichen. Später wurde jedoch der Abstand der Eisenlagen parallel zur Grenzfläche variiert, um eine bessere Übereinstimmung mit den gemittelten experimentellen Abbildungen zu erreichen. Der Atomabstand innerhalb dieser Lagen blieb dabei an die Gitterkonstante des $\mathrm{MgO}$ gekoppelt $\left(a_{\mathrm{Fe}}=\sqrt{2} \cdot a_{\mathrm{MgO}}\right)$. Eine mögliche Ursache für diese nicht volumenerhaltende Gitterverzerrung könnte zum Beispiel in den Tetraederlücken des Eisens eingelagertes Bor sein.

Die mittlere atomare Dichte $f_{i j}$ eines Atoms $j$ in der $i$-ten Lage ergibt sich nun durch Faltung der zugehörigen Gaußfunktion mit der mittleren atomaren Dichte der vorherigen Lage:

$$
\begin{aligned}
f_{i j}(\vec{r}) & =\frac{1}{(2 \pi)^{3 / 2} \sigma_{i}} \exp \left(-\frac{1}{2} \frac{\left(\vec{r}-\vec{r}_{i j}\right)^{2}}{\sigma_{i}^{2}}\right) \\
\text { mit } \sigma_{i} & =\sqrt{\sigma_{\mathrm{O}-\mathrm{Fe}}^{2}+(i-1) \sigma_{\mathrm{Fe}-\mathrm{Fe}}^{2}}
\end{aligned}
$$

Daraus folgt die zweidimensionale Verteilungsfunktion $\rho(x, y)$, indem die mittlere atomare Dichte aller Atome $f_{i j}$ in z-Richtung über die Höhe $c$ der Superzelle aufintegriert wird und anschließend aufsummiert wird.

$$
\rho(x, y)=\sum_{i} \sum_{j} \int f_{i j}(\vec{r}) d z
$$

\subsubsection{Elektrische Transportmessungen}

Die effizienteste Methode für elektrische Transportmessungen bildet der Einsatz einer Probestation. Mit der hier verwendeten Probestation der Firma SÜSS, wie sie in Abbildung 3.7 gezeigt ist, ist es möglich bis zu vier dünne Nadeln mittels Mikro-Manipulatoren beliebig auf einer Probe zu platzieren und diese an beliebigen Stellen flexibel zu kontaktieren. Auf diese Weise können die Proben mit 


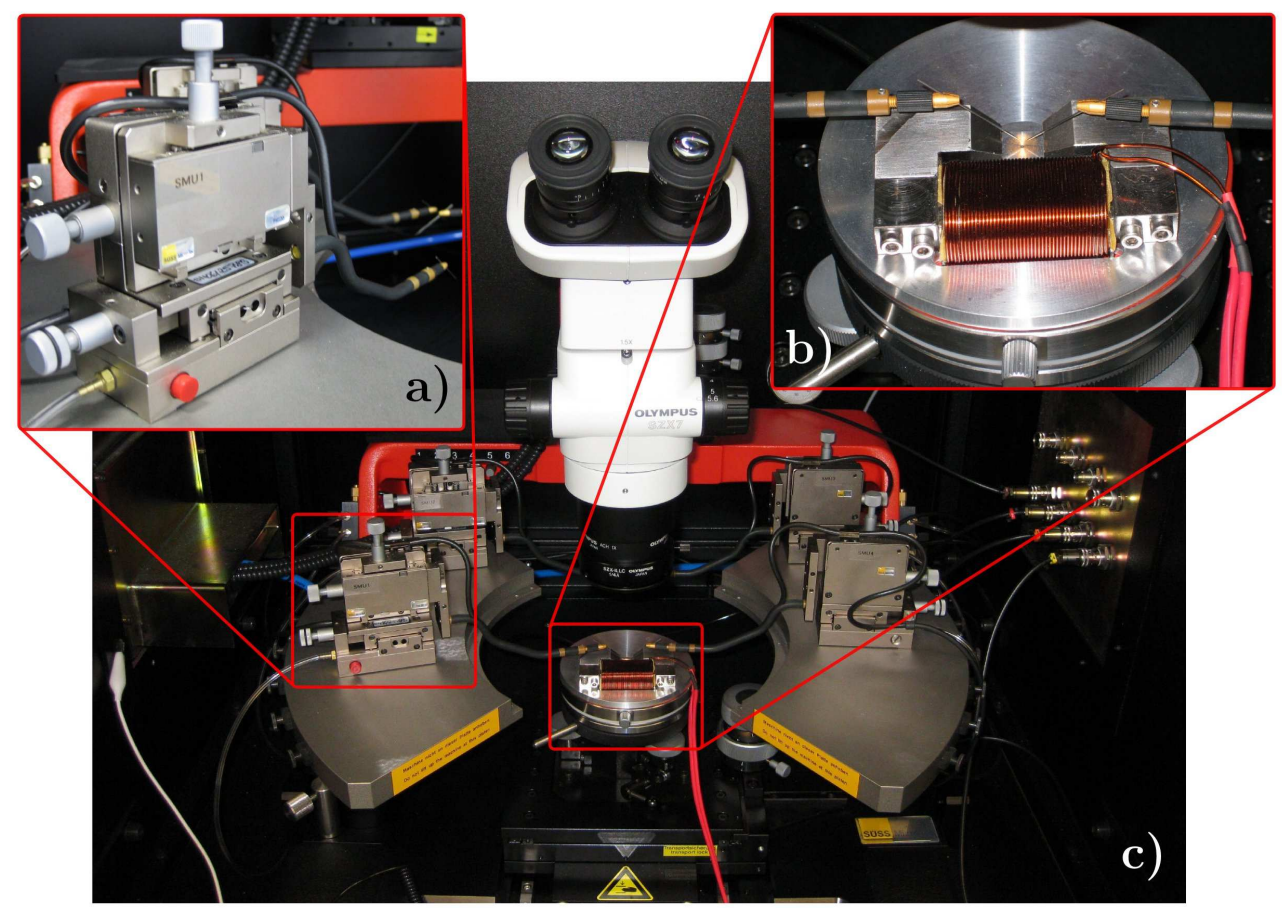

Abbildung 3.7: SÜSS MicroTec Probestation: a) Einer der Mikro-Manipulatoren, b) Probentisch mit Elektromagnet, c) Übersichtsbild. Die Aufnahme entstammt der Diplomarbeit von MARVIN WALTER $\underline{56}$

wenig Aufwand vermessen werden und es kann insbesondere auf aufwendige lithographische Prozesse zur Kontaktierung der Elemente verzichtet werden.

Die Mikro-Manipulatoren können auf den Manipulatortischen rechts und links vom Probentisch grob positioniert werden und werden in dieser Position mittels einer Vakuumpumpe fixiert. Die Feinpositionierung der Nadeln erfolgt dann mit drei Mikrometerschrauben, wie sie in der Großaufnahme Abbildung 3.7 a zu sehen sind. Zur optischen Kontrolle dient dabei ein Auflichtmikroskop, welches in Abbildung 3.7c in der Mitte zu sehen ist. Der Probentisch, der auch in allen drei Raumrichtungen beweglich ist, ist in Abbildung 3.7b mit zwei positionierten Sondennadeln gezeigt. Man erkennt hier auch den selbstgebauten Elektromagneten, der Magnetfeldabhängige Messungen mit Feldern von bis zu 100 mT ermöglicht.

Mit dieser Probestation sind Messungen sowohl in 2-Punkt- als auch in 4-PunktGeometrie durchführbar. Dabei eignet sich der verwendete Messaufbau für Gleichspannungsmessungen, wie $I(U)$-Kurven oder $R(H)$-Kurven, ebenso wie für inelas- 

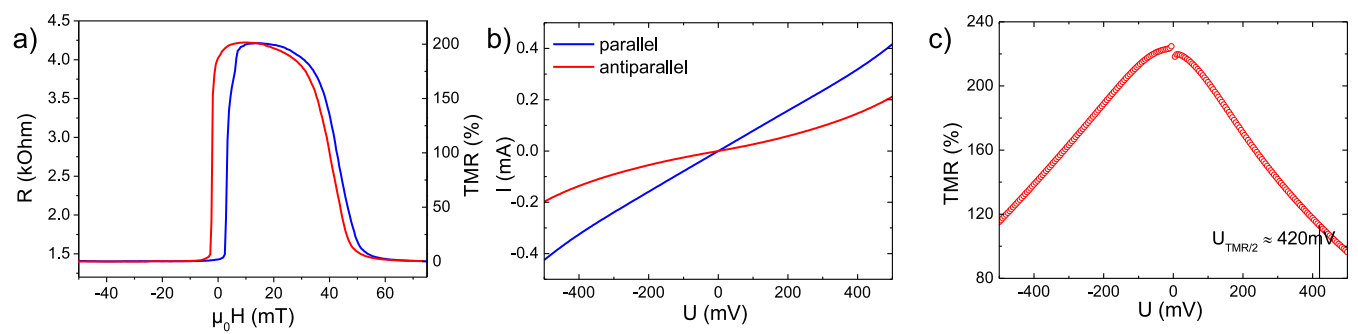

Abbildung 3.8: Beispiele für elektrische Transportmessungen an magnetischen Tunnelelementen: a) $\mathrm{R}(\mathrm{H})$, b) $\mathrm{I}(\mathrm{U})$ und c) $\mathrm{TMR}(\mathrm{U})$.

tische Tunnelspektroskopie durch Messungen von $\mathrm{d} I / \mathrm{d} U(U)$ oder $\mathrm{d}^{2} I / \mathrm{d} U^{2}(U)$ mithilfe eines Lock-In-Verstärkers. Letzteres ermöglicht es auch die Beiträge von Prozessen wie Phonenenanregungen oder Spin-Flip Streuungen zum Tunnelstrom $\mathrm{zu}$ isolieren. Einige Beispielmessungen zur Charakterisierung von magnetischen Tunnelelementen sind in Abbildung 3.8 gegeben.

Eine detaillierte Beschreibung der Transportmessungen und des verwendeten Messaufbaus ist in der Diplomarbeit von MARvin WALTER ${ }^{56} \mathrm{zu}$ finden. 



\section{Ergebisse}

Das Ergebniskapitel gliedert sich in drei Teile. Im ersten Teil wird ein Modellsystem der Grenzfläche zwischen kristallinem $\mathrm{MgO}$ und amorphem $\mathrm{CoFeB}$ vorgestellt, welches dann mit den Untersuchungen an vollständigen magnetischen Tunnelsystemen verglichen werden soll. Der letzte Teil zeigt die Auswirkungen von elektrischer Überlastung auf Tunnelelemente mit unterschiedlichen Strukturgrößen.

\subsection{Modellsystem MgO / CoFeB}

Zunächst wird in diesem Abschnitt die Kristallisation von CoFeB-Schichten unter optimalen Bedingungen untersucht. Hierzu wurde ein CoFeB-Film auf einkristallines MgO-Substrat gesputtert und anschließend bei verschiedenen Temperaturen für eine Stunde ausgelagert. Querschnitte dieser Proben wurden zunächst im Transmissionselektronenmikroskop untersucht und auf die erhaltenen hochaufgelösten Aufnahmen die im vorigen Kapitel vorgestellten Methoden der Mittelung angewandt. Ein Vergleich dieser Mittelungen mit Simulationen für ein angepasstes Modell bildet dann den Abschluss. Die Ergebnisse wurden in EILERS ET AL. 57 veröffentlicht.

\subsubsection{TEM-Untersuchungen auf der $\mu \mathrm{m}$-Skala}

In Abbildung 4.1 sind Übersichtsaufnahmen eines $280 \mathrm{~nm}$ dicken $\mathrm{Co}_{0.4} \mathrm{Fe}_{0.4} \mathrm{~B}_{0.2^{-}}$ Films auf $\mathrm{MgO}(100)$ nach verschiedenen Auslagerungstemperaturen dargestellt. Während für Temperaturen bis $340^{\circ} \mathrm{C}$ noch keine makroskopische Kristallisation $\mathrm{zu}$ erkennen ist, ist die Kristallisationsfront bei $400^{\circ} \mathrm{C}$ bereits $30-40 \mathrm{~nm}$ in den amorphen Film vorgedrungen. Dabei verläuft das Fortschreiten der Kristallisationsfront in die amorphe Schicht offensichtlich nicht homogen. Die Kristallisation startet an verschiedenen Nukleationspunkten und es sind immer noch schmale Bereiche an der Grenzfläche zu finden, welche nicht kristallisiert sind. Die Kristallitgröße kann auf 20-40 nm abgeschätzt werden. Während auch bei $400^{\circ} \mathrm{C}$ in der Mitte des Films keine nennenswerte Kristallisation zu erkennen ist, finden sich hier bei $425^{\circ} \mathrm{C}$ bereits Kristallite in der Größenordnung der Filmdicke. 

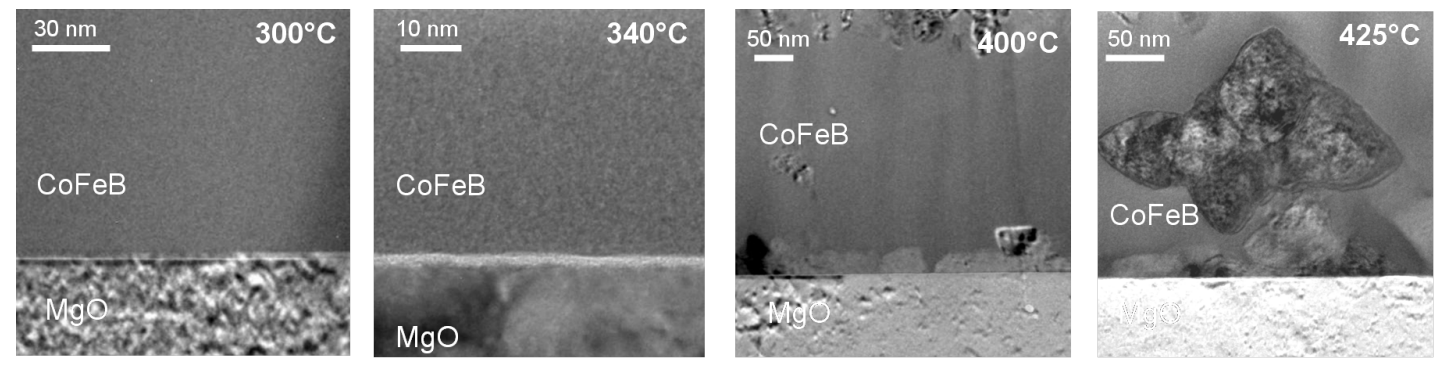

Abbildung 4.1: Übersichtsaufnahmen eines $280 \mathrm{~nm} \mathrm{Co} \mathrm{Co}_{0.4} \mathrm{Fe}_{0.4} \mathrm{~B}_{0.2}$-Films auf $\mathrm{MgO}(100)$ nach verschiedenen Auslagerungs-Temperaturen (postgrowth annealing für eine Stunde).

In einem magnetischen Tunnelelement ist weder ein Start der Kristallisationsfront von den Elektroden her noch eine Nukleation innerhalb des Films selbst erwünscht. Um einen hohen TMR zu erzielen ist es eine Voraussetzung, das die Kristallstruktur des Ferromagneten in der Nähe der Grenzfläche vom MgO induziert wird (solid phase epitaxy). Daher wird im folgenden betrachtet, inwiefern sich die inhomogene Kristallisation auf der Subnanometerskala widerspiegelt.

\subsubsection{Hochaufgelöste Transmissionselektronenmikroskopie}

Um bei der Mittelung von TEM Abbildungen, wie sie in Abschnitt 3.5 beschrieben wurde, Ergebnisse mit ausreichender statistischer Signifikanz zu erzielen, ist es erforderlich, über eine ausreichende Anzahl an Streifen zu mitteln. Andererseits ist es insbesondere bei kompletten TMR-Elementen oft schwierig, ausreichend große Bereiche mit entsprechend glatter Grenzfläche zu finden. In Abbildung 4.2 sind vier verschiedene Bereiche mit einer Breite von 15 Streifen entlang der Grenzfläche für die Probe mit einer Annealingtemperatur von $300^{\circ} \mathrm{C}$ und darunter das Ergebnis der jeweiligen Mittelung dargestellt. Es sind deutlich Merkmale kristalliner Muster in den ersten Lagen des $\mathrm{CoFeB}$ zu erkennen, die in ihren Details in den vier gewählten Mittelungsbereichen unterschiedlich sind. Da Abbildungsbedingungen und der Probendicke in Strahlrichtung in den gewählten Mittelungs- 

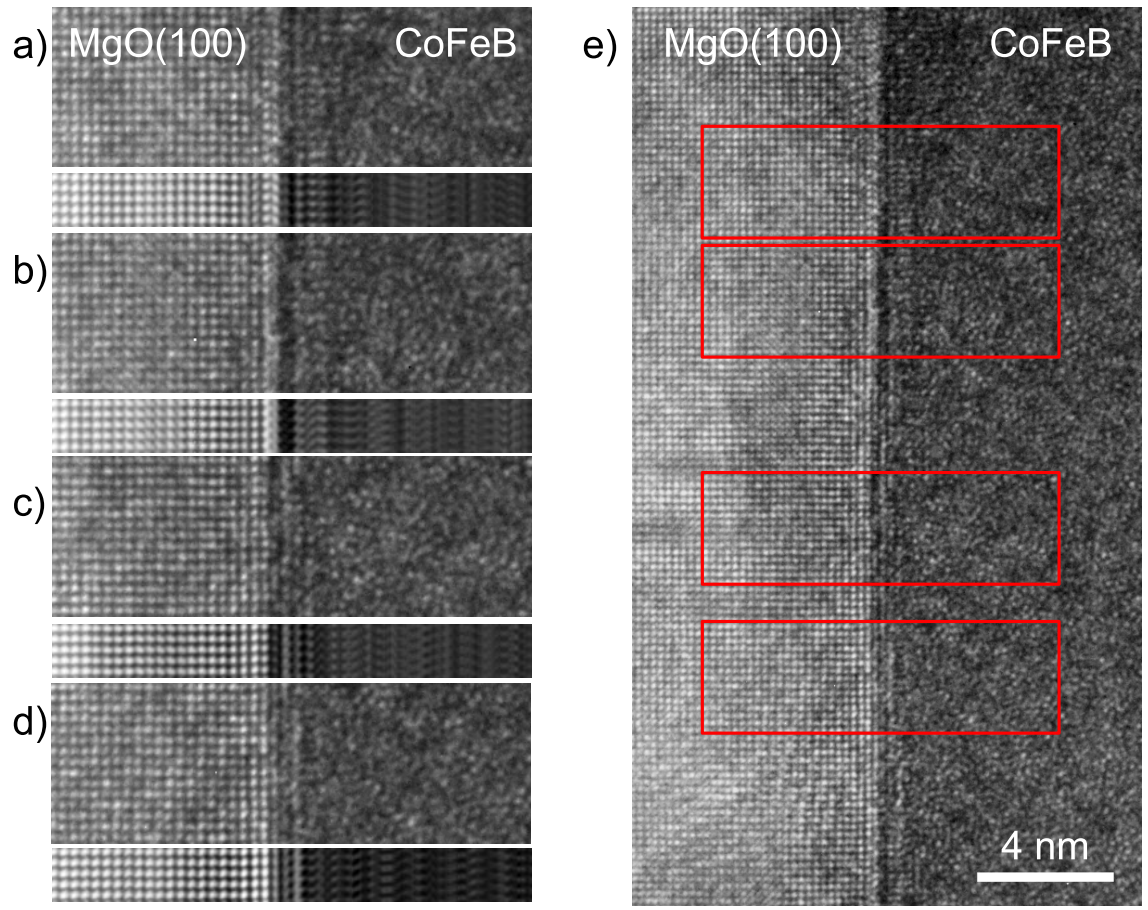

Abbildung 4.2: Mittelungen über 15 Streifen für unterschiedliche Bereiche der Grenzfläche der Probe mit einer Annealingtemperatur von $300^{\circ} \mathrm{C}$. In der Übersicht (rechts) sind die Mittelungsbereiche rot markiert. Auf der linken Seite sind die vergrößerten Mittelungsbereiche und darunter das Ergebnis der Mittelung dargestellt.

bereichen nur wenig unterschiedlich sind, deutet dies auf strukturelle Variationen entlang der Grenzfläche hin.

Um die Auswirkungen des Mittelungsbereiches zu verdeutlichen, sind in Abbildung 4.3 zwei aneinander grenzende Bereiche von 15 Streifen Breite mit der jeweiligen Mittelung und zusätzlich die Mittelung über alle 30 Streifen dargestellt. Die Untergrenze des Bereiches stimmt dabei mit der Untergrenze des vierten Bereiches inAbbildung 4.2 d) überein. Man erkennt deutlich, dass die Kontrastschwankungen mit zunehmender Mittelungsbreite in den Hintergrund treten und die Merkmale der zweidimensionalen Atomverteilung deutlicher erkennbar werden. Für die Vergleiche mit simulierten Abbildungen wird im Folgenden eine Mittelung über 121 Streifen, entsprechend den gesamten in Abbildung 4.2 rechts dargestellten Bereich, verwendet. 


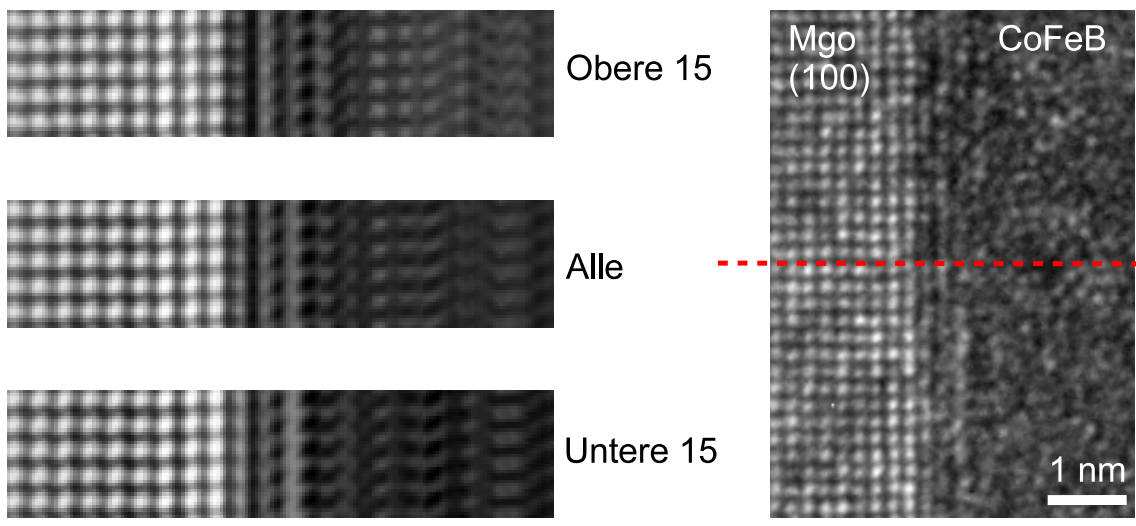

Abbildung 4.3: Vergleich der Mittelungsbreite. Links sind die Ergebnisse der Mittelung über den gesamten rechts dargestellten Mittelungsbereich und zum Vergleich für die obere und untere Hälfte gezeigt.

\subsubsection{Vergleich mit Simulationen}

Da Eisen und MgO pseudomorph aufeinander wachsen können, ist es eine Frage der untersuchten Problemstellung, wie sich dabei die Gitterfehlpassung auflöst. Dies hat zur Folge, dass die Positionen der Magnesium- und Sauerstoffatome in der Superzelle dadurch bestimmt wird, ob das $\mathrm{MgO}$ verspannt auf dem Eisen aufwächst oder umgekehrt. Außerdem gibt es bei der Durchstrahlung parallel zur Grenzfläche zwei mögliche Richtungen hoher Symmetrie im MgO. In Abbildung 4.4 sind simulierte Abbildungen unterschiedlich dicker MgO-Schichten für Durchstrahlrichtungen entlang der (001)- und der (011)-Richtung gezeigt, jeweils für verspanntes $\mathrm{MgO}$ mit einer Gitterkonstante, welche sich von der des Eisens ableitet, und für unverspanntes $\mathrm{MgO}$ mit der Gitterkonstante des Volumenkristalls. Unter den Abbildungen ist das zugehörige projizierte Potential dargestellt. Es wird schnell deutlich, dass die Sauerstoffatome kaum zur Kontrastentstehung beitragen. Im Fall des im vorigen Abschnitt gezeigten Modellsystems, CoFeB auf $\mathrm{MgO}$, entsprechen die Abbildungen denen der zweiten Reihe in Abbildung 4.4 für unverspanntes $\mathrm{MgO}$ in (001)-Richtung. In diesem Fall hat auch die Probendicke den geringsten Einfluss auf den entstehenden Kontrast.

Für die Simulation elektronenmikroskopischer Abbildungen ist neben der Atomverteilung auch die Vorgabe der optischen Parameter des verwendeten Mikroskps erforderlich. Da für die Abbildungen in dieser Arbeit dasselbe Phillips CM200UT FEG Mikroskop wie in der Arbeit von KARsten ThIEL ${ }^{18}$ benutzt wurde, wurden auch diese Parameter von ihm übernommen. Aus der Beschleunigungs- 


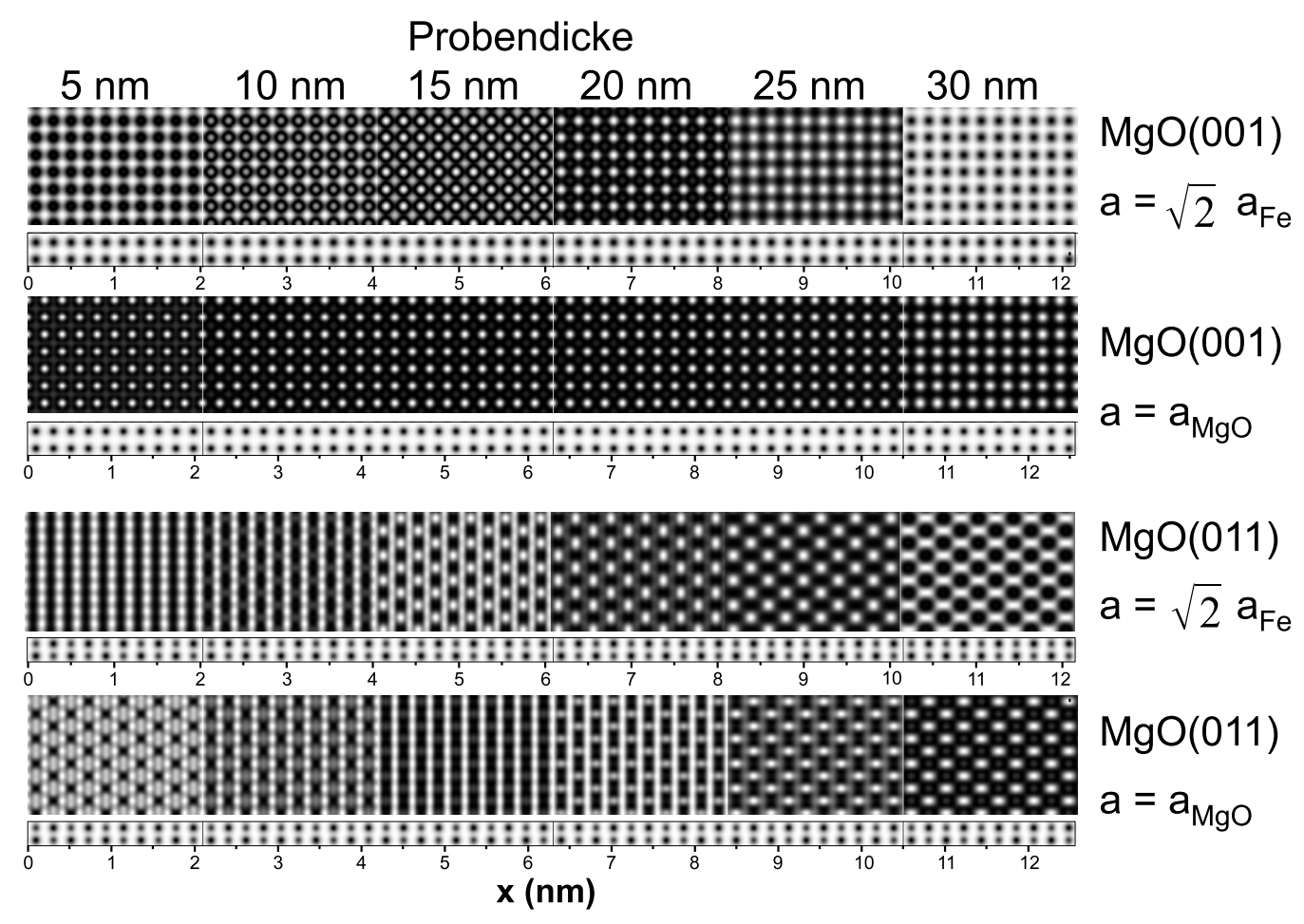

Abbildung 4.4: Einfluss der Probendicke und der Gitterkonstante auf die simulierten Abbildungen von kristallinem $\mathrm{MgO}$, hier dargestellt für einen Defokus $\Delta f=17 \mathrm{~nm}$. Dargestellt ist jeweils oben die simulierte Abbildung und darunter das projizierte Potential für Probendicken von $5 \mathrm{~nm}$ bis $30 \mathrm{~nm}$. In den oberen beiden Reihen ist die Durchstrahlrichtung parallel zur $\mathrm{MgO}(001)$-Richtung, in den unteren Reihen parallel zur $\mathrm{MgO}(011)$-Richtung. Die jeweils erste Reihe zeigt ein verzerrtes $\mathrm{MgO}$ mit einer vom Eisen abgeleiteten Gitterkonstante $a=\sqrt{2} * a_{F e}=0,406 \mathrm{~nm}$, die zweite Reihe zeigt unverzerrtes $\mathrm{MgO}$ mit einer Gitterkonstante $a=0,421 \mathrm{~nm}$.

spannung von $200 \mathrm{kV}$ folgt eine Wellenlänge $\lambda=2,508 \mathrm{pm}$, die sphärische Aberation beträgt $C_{s}=0,49 \mathrm{~mm}$, die Defokusstreuung ist $\Delta=4,0 \mathrm{~nm}$ und der Semikonvergenzwinkel der Beleuchtung ist $\alpha=0,2 \mathrm{mrad}$. Desweiteren ist noch die Vorgabe der Probendicke und des Defokus nötig. Die Probendicke beeinflusst die Austrittswellenfunktion der Elektronen und die Defokussierung bestimmt das Übertragungsverhalten des Mikroskops. Abbildung 4.5 zeigt das Vorgehen bei der Ermittlung der geeignetsten Parameter durch einen iterativen Bildvergleich. Da in einem kristallinen Material die Atompositionen bekannt sind, werden für die Anpassung die Werte über einen weiten Parameterraum variiert und zugehörige 

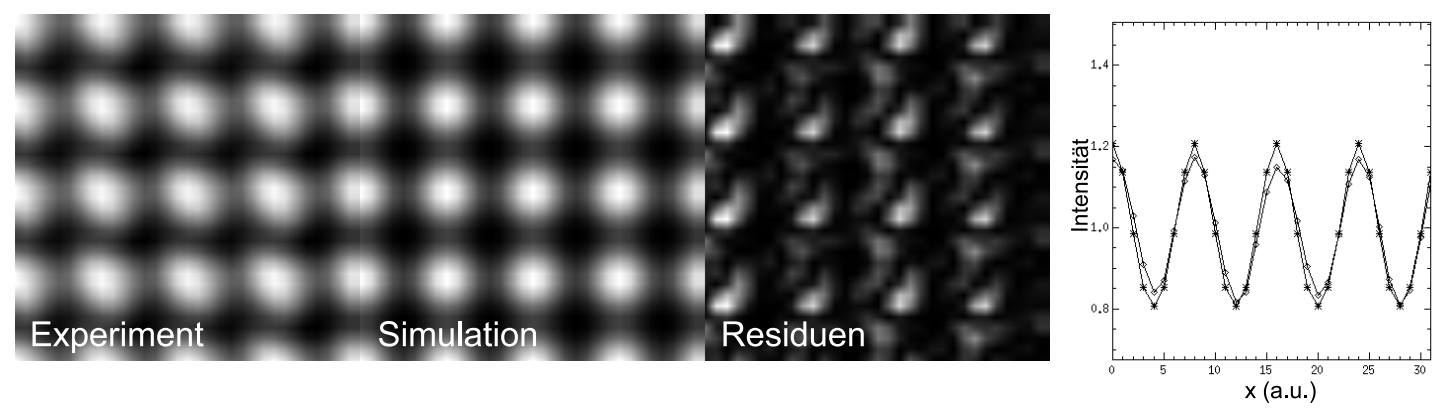

Abbildung 4.5: Bestimmung von Probendicke und Defokus durch Minimierung der Differenz zwischen experimenteller und simulierter Abbildung. Die beste Anpassung ergibt sich für eine Dicke von $21 \mathrm{~nm}$ und einen Defokus $\Delta f=-46 \mathrm{~nm}$ bzw. $\Delta f=$ $-81 \mathrm{~nm}$.

simulierte Abbildungen für kristallines MgO berechnet. Ein Vergleich der simulierten Abbildungen mit der experimentellen Abbildung des MgO-Teils der Probe, durch Bestimmung eines Differenzmaßes für die Abweichungen in den Intensitäten, führt dann zu dem Parametersatz, für den die bestmögliche Anpassung erreicht wird.
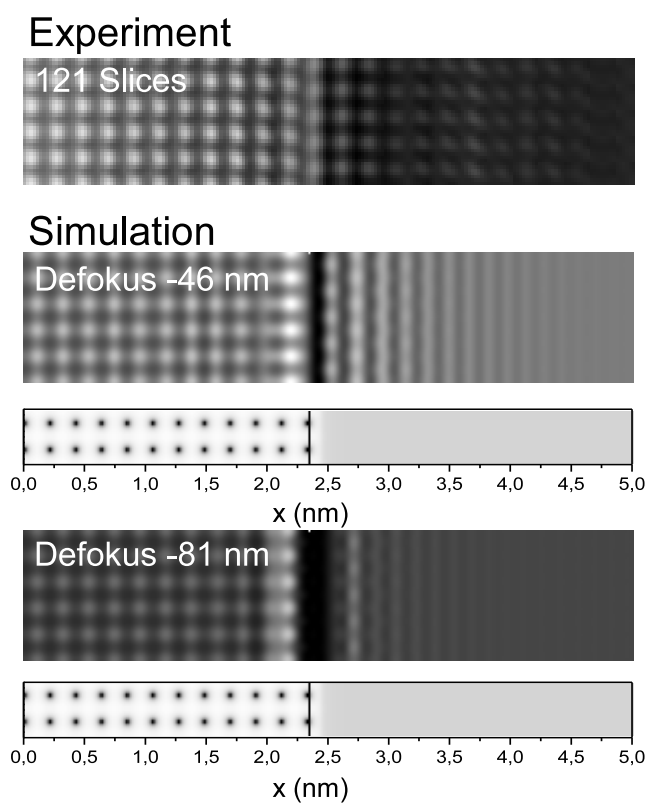

Abbildung 4.6: Vergleich der gemittelten experimentellen Abbildung mit simulierten abbildungen Abbildungen für $21 \mathrm{~nm}$ Probendicke und einen Defokus von $\Delta f=-46 \mathrm{~nm}$ (Mitte) bzw. $\Delta f=-81 \mathrm{~nm}$ (unten). Unterhalb der simulierten Abbildungen ist das projizierte Potential mit einer Markierung der letzten Lage $\mathrm{MgO}$ dargestellt. Die Skala gibt einen Abstand in $\mathrm{nm}$ mit willkürlichem Nullpunkt an.

Abbildung 4.6 zeigt zwei Simulationen für eine Probendicke von $d=21 \mathrm{~nm}$ und 
die Defoki $\Delta f=-46 \mathrm{~nm}$ und $\Delta f=-81 \mathrm{~nm}$, für welche sich die beste Anpassung ergab. Für den amorphen Bereich wurde hierbei eine homogene Verteilung von Eisen eingesetzt, deren atomare Dichte der von kristallinem Eisen entspricht. Die homogene Verteilung setzt dabei in einem Abstand von der letzten Lage MgO ein, der dem halben Abstand zwischen $\mathrm{MgO}$ und kristallinem Eisen bei epitaktischem Wachstum entspricht, welcher mit $a_{\mathrm{O}-\mathrm{Fe}}=2,35 \AA$ aus der Literatur entnommen wurde 55. Dargestellt ist jeweils der gemittelte Streifen, welcher zur besseren Darstellung mehrmals untereinander wiederholt wird, und darunter das projizierte Potential. Der senkrechte Strich im projizierten Potential verdeutlicht die Position der letzten Lage kristallinen MgOs.

Bei dem Vergleich von gemittelten experimentellen und simulierten Abbildungen darf man sich nicht von der Stärke des Kontrastes fehlleiten lassen. Bei gegebener Atomverteilung ist es normal, dass simulierte Abbildungen einen dreifach bis fünffach höheren Kontrast aufweisen als experimentelle. Dieser Umstand wird in der Literatur als Stobbs-Faktor ${ }^{58 / 59}$ bezeichnet, dessen Ursachen zum Beispiel bei THIEL ${ }^{18}$ näher erläutert werden. Der Vergleich darf sich also nur auf charakteristische Merkmale in der Struktur der Atomverteilung stützen. Für einen softwaregestützten quantitativen Vergleich der Intensitäten von simulierten und experimentellen Abbildungen wäre hier zunächst eine Normierung nötig, um den Stobbs-Faktor auszugleichen.

Abbildung 4.7 zeigt einen Vergleich zwischen der Mittelung über 121 Streifen mit drei verschiedenen zweidimensionalen Verteilungsfunktionen nach dem in $\mathrm{Ab}$ schnitt 3.2.4 beschriebenen Verfahren. Die Simulationen unterscheiden sich in der Bindungswinkelvariation von $5^{\circ}, 7^{\circ}$ und $9^{\circ}$. Bei der Betrachtung des projizierten Potentials wird schnell die Auswirkung der Bindungswinkelvariation auf die zweidimensionale Verteilungsfunktion deutlich: Die Reichweite der grenzflächeninduzierten Variation in der Verteilungsfunktion im amorphen $\mathrm{CoFeB}$ ist für $\varphi=5^{\circ}$ bis sechs Lagen und für $\varphi=9^{\circ}$ bis zwei Lagen sichtbar. Unabhängig vom stärkeren Kontrast der simulierten Abbildungen lässt sich ein charakteristisches Detail sowohl in der experimentellen als auch in den gemittelten Abbildungen erkennen. In beiden Fällen zeigt der Kontrast der atomaren Reihen einen Sprung um einen halben Atomlagenabstand direkt an der Grenzfläche, obwohl dieser Versatz in den zugrundeliegenden Atomverteilungen, wie auch im projizierten Potential erkennbar, nicht vorhanden ist. In Abbildung 4.7 ist dieser Sprung im Kontrast durch die gepunkteten roten Linien hervorgehoben. Dass dieser Effekt auf die Struktur des Eisens in der Nähe der Grenzfläche zurückzuführen ist, zeigt sich auch daran, dass ein derartig verschobener Kontrast in den Simulationen mit einer homogenen Eisenverteilung im amorphen Teil (vergl. Abbildung 4.6) nicht auftritt.

In der Literatur wird häufig berichtet, dass der TMR durch die Bildung einer FeO-Grenzschicht negativ beeinflusst wird. Die experimentellen Befunde die- 

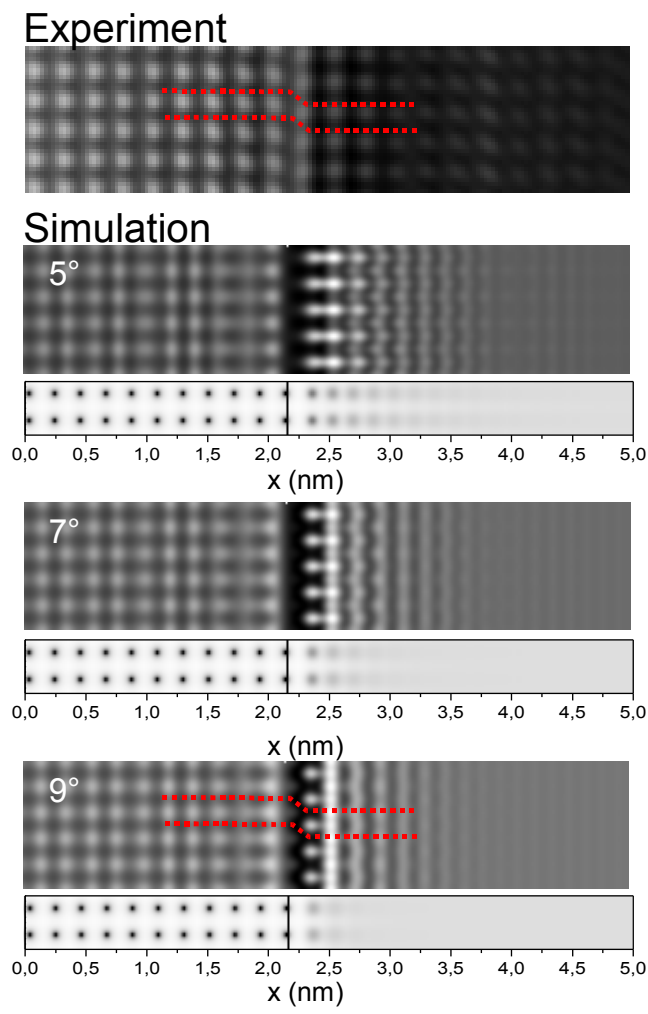

Abbildung 4.7: Vergleich einer Mittelung über 121 Streifen mit simulierten Abbildungen für $5^{\circ}, 7^{\circ}$ und $9^{\circ}$ Bindungswinkelvariation. Die rote Markierung verdeutlicht den Sprung im Kontrast an der Grenzfläche um eine halbe Atomlage.

ses Verhaltens sowie die theoretischen Modelle zu seiner Erklärung wurden in Abschnitt 2.2.3 erläutert. An dieser Stelle soll überprüft werden, ob eine solche FeO-Zwischenschicht auch durch den Vergleich von gemittelten experimentellen und simulierten Abbildungen gefunden werden kann. Abbildung 4.8 zeigt einen Vergleich zwischen derselben Mittelung über 121 Streifen wie in Abbildung 4.7 mit drei verschiedenen zweidimensionalen Verteilungsfunktionen mit einer festen Bindungswinkelvariation von $7^{\circ}$ bei denen an der Grenzfläche eine Lage FeO mit unterschiedlichen Sauerstoffsättigungen eingefügt wurde. Die simulierten Abbildungen unterscheiden sich zwar in der Kontraststärke von denen ohne FeO, nicht aber in dem charakteristischen Sprung in den Atomreihen und den Atomabständen sowie der Reichweite der kristallinen Ordnung. Da der Kontrast der simulierten Abbildungen schon aufgrund des Stobbsfaktors von dem der experimentellen Abbildung abweicht, ist der Effekt des Sauerstoffs an der Grenzfläche hier schwer zu separieren.

Bei den Simulationen hat sich gezeigt, dass der oben erwähnte Kontrastsprung an der Grenzfläche in die Eisenelektrode hinein verschoben wird, wenn die Bindungswinkelvariation als Parameter für die Erzeugung der zweidimensionalen Verteilungsfunktion sehr klein gewählt wird. In Abbildung 4.9 sind simulierte Abbil- 

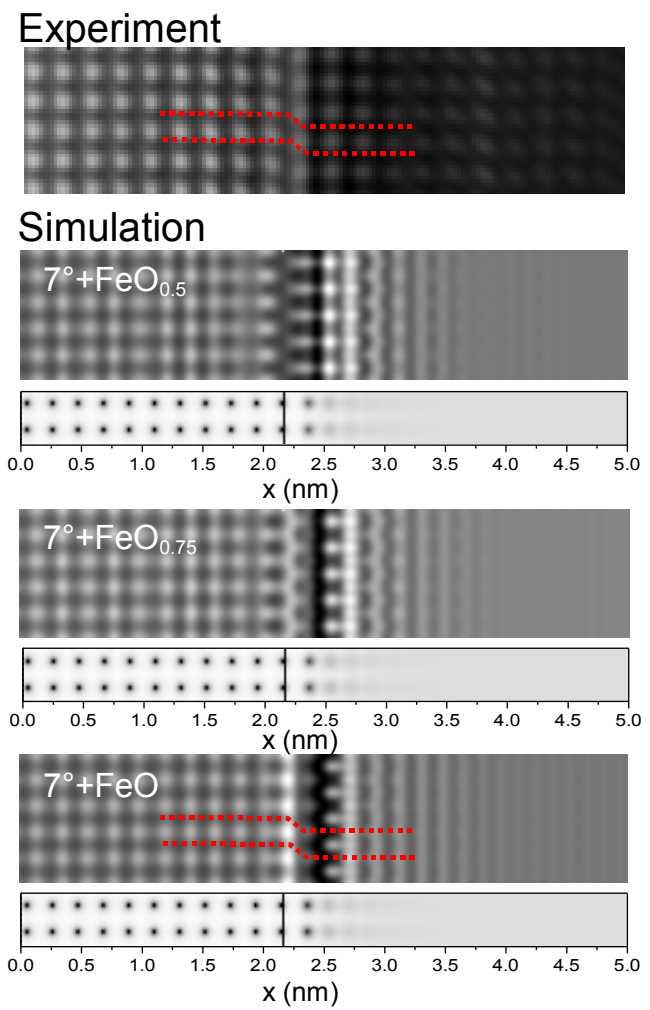

Abbildung 4.8: Vergleich einer Mittelung über 121 Streifen mit simulierten Abbildungen für $7^{\circ}$ Bindungswinkelvariation und einer an der Grenzfläche eingefügten Lage $\mathrm{FeO}$ mit unterschiedlicher Sauerstoffsättigung. Die rote Markierung verdeutlicht den Sprung im Kontrast an der Grenzfläche um eine halbe Atomlage.

dungen für sehr kleine Bindungswinkelvariationen von $0,7^{\circ}$ bis $2^{\circ}$ und das jeweils zugehörige projizierte Potential gezeigt, um diesen Effekt zu verdeutlichen. Im projizierten Potential ist für die grenzflächennahen Lagen des Eisens keine nennenswerte Verbreiterung der Atome zu erkennen, es unterscheidet sich visuell nicht von einer kristallinen Atomverteilung. Dennoch ist die mittlere Atomdichte im Zentrum der Atomsäulen in der zweidimensionalen Verteilungsfunktion deutlich gegenüber der einer kristallinen Atomverteilung reduziert und dadurch auch das Streupotential der Probe an dieser Stelle verändert. Auf die Abbildung hat dies einen ähnlichen Effekt, wie die Reduzierung der Probendicke. Auch die Variation der Probendicke wirkt sich auf die Abbildung aus und kann bei geeigneten Werten zu einer völligen Kontrastumkehr führen, wie es in Abbildung $4.4 \mathrm{am}$ Beispiel von $\mathrm{MgO}$ zu erkennen ist. Die zunehmende Verbreiterung der mittleren Atomdichte von Lage zu Lage im amorphen Eisen kann also auch als lokale Reduzierung der effektiven Probendicke betrachtet werden und ab einer bestimmten Verbreiterung erfolgt die Kontrastumkehr. Aus der Konstruktionsvorschrift für die zweidimensionale Verteilungsfunktion folgt dann für die Lagennummer in der der Kontrastsprung auftritt $i_{/ \text {Sprung }}=\propto(\tan \varphi)^{-2}$. In Abbildung 4.4 erkennt man für $2^{\circ}$ Bindungswinkelvariation gerade noch eine Lage im Eisen, die gegenüber den 

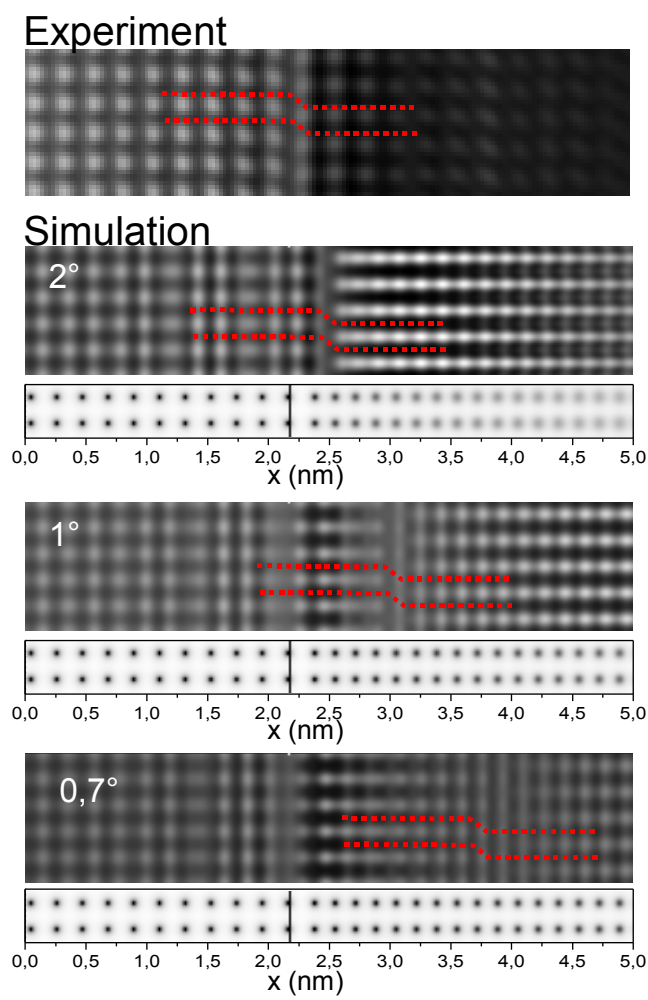

Abbildung 4.9: Abhängigkeit des Kontrastsprungs von der Aufweitung der zweidimensionalen Verteilungsfunktion und zum Vergleich die Mittelung über 121 Streifen. Für kleine Bindungswinkelvariationen verschiebt sich der Kontrastsprung in das amorphe Material, hier gezeigt anhand der simulierten Abbildungen für $2^{\circ}, 1^{\circ}$ und $0,7^{\circ}$. Die rote Markierung verdeutlicht den Sprung im Kontrast um eine halbe Atomlage.

Atomreihen im $\mathrm{MgO}$ nicht verschoben ist, für $1^{\circ}$ Bindungswinkelvariation erfolgt der Kontrastsprung nach der vierten Lage und für $0,7^{\circ}$ etwa nach der neunten, die quadratische Abhängigkeit der Position des Kontrastsprungs von der Bindungswinkelvariation scheint also erfüllt zu sein.

Bemerkenswert ist, dass die Halbwertsbreite der Gaußverteilung der mittleren Atomdichte an der Stelle des Kontrastsprungs nur ca. 9\% des Abstandes nächster Nachbarn im Eisengitter entspricht. Simulationen mit Variation der Probendicke zeigen, dass eine Kontrastumkehr im Eisen und $\mathrm{MgO}$ gleichermaßen auftritt. Tritt in einer gemittelten experimentellen Abbildung ein Sprung im Kontrast an der Grenzfläche um eine halbe Atomlage auf, was auf eine Kontrastumkehr in nur einem der beiden Materialien hindeutet, so ist das ein Indiz dafür, dass schon die ersten Lagen der ferromagnetischen Elektrode vom kristallinen Gitter abweichen.

\subsection{Untersuchungen an realen Devices}

Die im vorigen Abschnitt angewandte Mittelungsmethode soll nun auf reale TMRStrukturen übertragen werden, um die vorhandene Grenzflächenordnung mit den 
erzielten Magnetowiderständen vergleichen zu können. Es handelt sich hierbei um ein sogenanntes Pseudo-Spinvalve (PSV), dies bedeutet, dass neben den beiden ferromagnetischen Elektroden keine weiteren magnetischen Schichten vorhanden sind, wie sie in anderen Systemen, zum Beispiel zur besseren magnetischen Separierung der Elektroden durch Austauschkopplung an einen Antiferromagneten, eingesetzt werden. Ein PSV ist also das einfachst mögliche TMR-System. Es werden drei unterschiedliche PSVs vorgestellt, welche sich in den Präparationsbedingungen unterscheiden, und deren Auswirkungen auf Struktur und Transporteigenschaften untersucht.

\subsubsection{Teilkristallines Pseudo-Spinvalve}

Der hier vorliegenden Fall zeigt ein PSV mit dem Schichtaufbau $\mathrm{SiO}_{x} / 5 \mathrm{~nm}$ $\mathrm{Ta} / 30 \mathrm{~nm} \mathrm{Ru} / 10 \mathrm{~nm} \mathrm{Ta} / 10 \mathrm{~nm} \mathrm{Ru} / 4 \mathrm{~nm} \mathrm{CoFeB} / 2,1 \mathrm{~nm} \mathrm{MgO} / 1,5 \mathrm{~nm} \mathrm{CoFeB} / 5 \mathrm{~nm}$ $\mathrm{Ta} / 20 \mathrm{~nm} \mathrm{Ru}$. Alle Schichten wurden durch Magnetronsputtern hergestellt und der gesamte Schichtstapel wurde anschließend bei $400^{\circ} \mathrm{C}$ für eine Stunde ausgelagert. Eine elektronische $\mathrm{R}(\mathrm{H})$-Messung ergibt einen Tunnelmagnetowiderstand von $210 \%$ bei einer Spannung von $10 \mathrm{mV}$. Abbildung 4.10 zeigt auf der rechten Seite eine HRTEM-Aufnahme eines Ausschnitts der Barriere sowie unten links eine zum selben Element gehörende R(H)-Messung. Da die Barriere und damit auch die Grenzflächen etwas rauer sind als dies beim Modellsystem mit einkristallinem Substrat der Fall ist, gestaltet es sich auch schwieriger große Bereiche mit homogen kristalliner Barriere und atomar flacher Grenzfläche zu finden. Die in Abbildung 4.10 links gezeigten Mittelungen konnten daher nur über Bereiche mit einer Breite von 15 Streifen ausgeführt werden. Die Bereiche, über welche die Mittelungen vorgenommen wurden, sind durch die roten Markierungen angedeutet.

Für die beiden Grenzflächen zur oberen bzw. unteren Elektrode wurden getrennte Mittelungen erzeugt, bei denen der Prozess der Streifenbildung an der jeweiligen Grenzfläche ausgerichtet wurde. In Abbildung 4.11 sind die Ergebnisse der Mittelung für ausgewählte Bereiche der oberen und unteren Grenzfläche noch einmal vergrößert dargestellt. Die auf der rechten Seite dargestellte Grenzfläche zur unteren Elektrode entspricht dem Mittelungsbereich b) in Abbildung 4.10. die auf der linken Seite dargestellte obere Grenzfläche entspricht dem Bereich zwischen a) und b) aus Abbildung 4.10. Zur besseren Veranschaulichung ist der gemittelte Streifen jeweils wieder mehrmals untereinander wiederholt und zusätzlich wurde in der jeweils unteren Hälfte der Mittelung auf dem amorphen Teil mit dem Bildbearbeitungsverfahren der Kontrastspreizung der Kontrastunterschied zur Barriere ausgeglichen. In beiden Fällen findet sich wieder der typische Kontrastsprung entlang der Atomreihen direkt an der Grenzfläche, verdeutlicht durch 
die roten Balken. Der Bereich der Barriere zeigt dabei deutlich die kristalline Struktur des MgOs mit einer (001)-Richtung in Durchstrahlrichtung. Die Mittelung über so wenige Streifen wie hier lässt sicher nur in sehr begrenztem Umfang quantitative Aussagen zu, dennoch kann für die untere Grenzfläche eine Reichweite der kristallinen Ordnung von mehr als drei Monolagen ausgeschlossen werden. Anders verhält es sich mit der Grenzfläche zur oberen Elektrode, hier lässt sich noch im Abstand von etwa sieben Atomlagen eine deutlich inhomogene Kontrast-

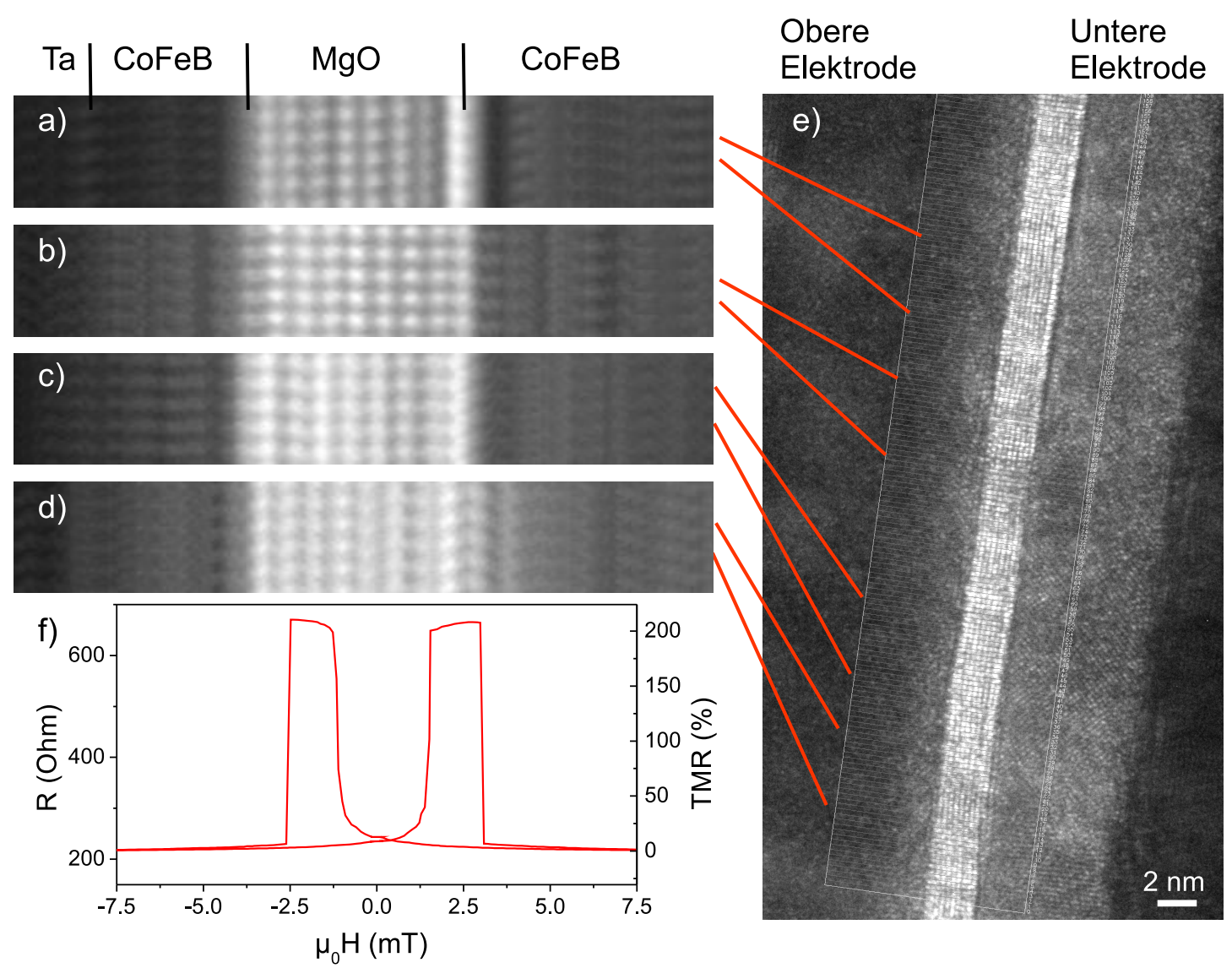

Abbildung 4.10: HRTEM-Aufnahme (e) eines Pseudo-Spinvalve Systems mit 210\% TMR und die Ergebnisse der Mittelungen (a-d) über verschiedene Bereiche der Barriere mit je 15 Streifen Breite. In allen Bereichen ist die (100)-Orientierung des MgOs deutlich zu erkennen. Zugehörige Magnetowiderstandsmessung (f). 

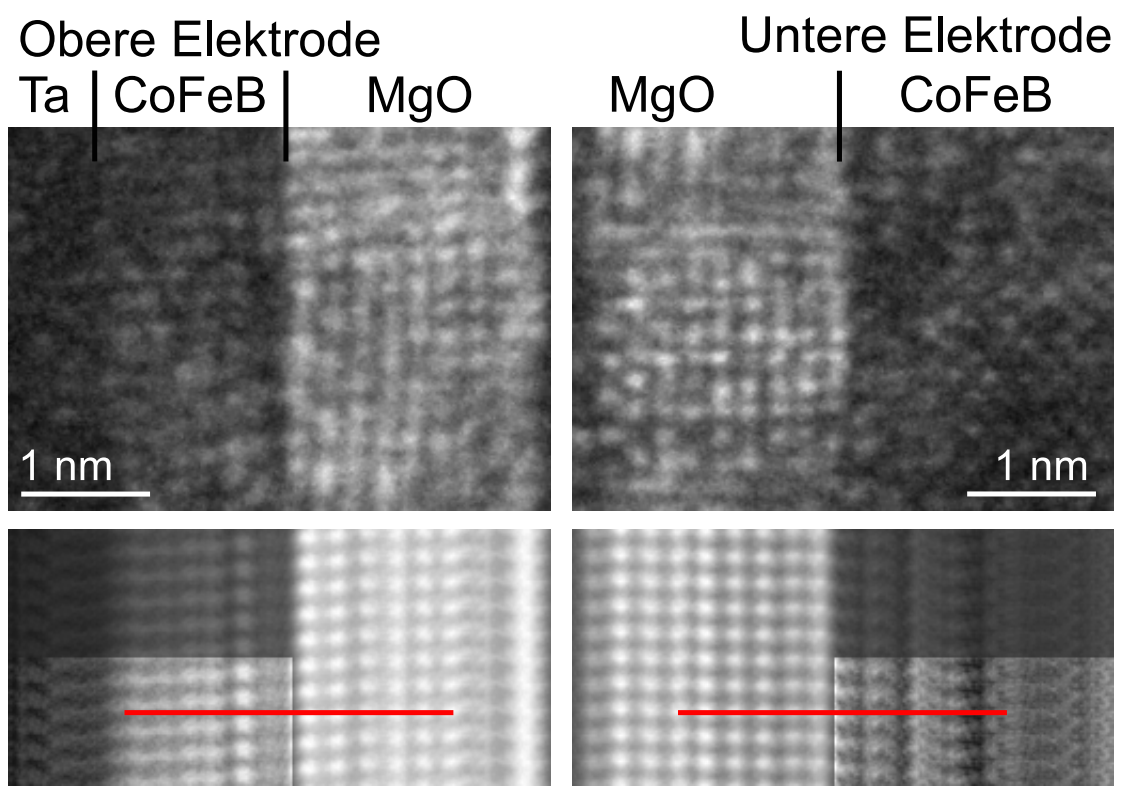

Abbildung 4.11: Mittelungsbereiche und gemittelte experimentelle Abbildung für die Grenzfläche zur oberen Elektrode (links, entspricht Bereich zwischen a) und b) aus Abbildung 4.10 und unteren Elektrode (rechts, entspricht Bereich b) aus Abbildung 4.10. Im unteren Teil der gemittelten Abbildung wurde die Intensität im amorphen Bereich zur Verdeutlichung reskaliert.

verteilung erkennen. Da dies auch der nominellen Schichtdicke des oberen CoFeB entspricht und danach ein neuerlicher Sprung in der Kontraststärke zu finden ist, der den Übergang zum Tantal markiert, kann für die obere Elektrode zumindest nicht ausgeschlossen werden, dass diese vollständig kristallisiert ist.

\subsubsection{Pseudo-Spinvalve mit thermisch zerstörtem TMR}

Das in Abbildung 4.12 dargestellte PSV-System unterscheidet sich von den im vorigen Abschnitt nur durch die höhere Auslagerungstemperatur von $500^{\circ} \mathrm{C}$. Dies führt aber zu drastischen Änderungen der Transporteigenschaften: Während sich der Flächenwiderstand etwa verzehnfacht, verschwindet der TMR vollständig. Die elektronenmikroskopischen Aufnahmen zeigen ebenfalls deutliche Änderungen gegenüber der bei $400^{\circ} \mathrm{C}$ ausgelagerten Probe. So sind oberhalb der Barriere keine getrennten Schichten mehr erkennbar. In der unteren CoFeB-Elektrode sind hingegen schon große kristalline Bereiche auszumachen. Dies wird auch durch die 


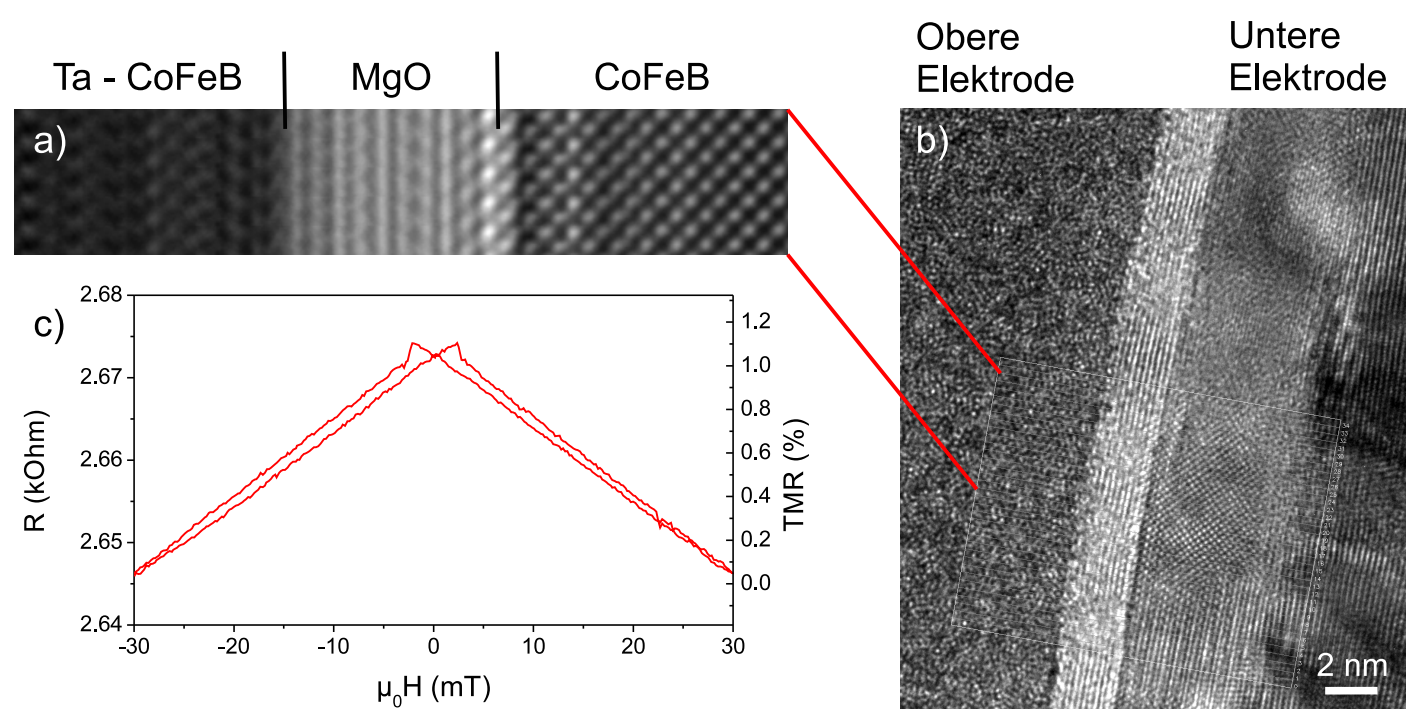

Abbildung 4.12: HRTEM-Aufnahme (b) eines Pseudo-Spinvalve-Systems ohne TMR nach Auslagerung bei $500^{\circ} \mathrm{C}$ und die Ergebnisse der Mittelung (a) über einen Teilbereich der Barriere mit 15 Streifen Breite. Es zeigt sich deutlich die (011)-Orientierung des MgOs, die sich in die untere Elektrode fortsetzt. Zugehörige Magnetowiderstandsmessung (c).

gezeigte Mittelung über einen Teilbereich mit einer Breite von 15 Streifen bestätigt. Die untere Elektrode zeigt durchgehend kristalline bcc-Struktur mit der (001)-Richtung als Durchstrahlrichtung. Der eher streifenartige Kontrast der Barriere steht nicht im Widerspruch zu einem pseudomorphen Wachstum, was bei einem Vergleich mit den in Abbildung 4.4 gezeigten Simulationen für $\mathrm{MgO}$ in (011)-Richtung deutlich wird. In den ersten beiden Lagen der Barriere ist die (011)-Richtung des MgOs auch noch deutlich zu erkennen. In der oberen Elektrode ist hingegen weder eine korrelierte kristalline Struktur zu erkennen noch sind irgendwelche Kontraständerungen nach der nominellen Dicke der CoFeB-Schicht auszumachen.

\subsubsection{Vergleich der Pseudo-Spinvalve-Systeme}

Diese Beobachtungen deuten bereits darauf hin, dass es in der oberen Elektrode durch die Auslagerung bei $500^{\circ} \mathrm{C}$ zu Veränderungen gekommen ist, welche den TMR zerstören. In Abbildung 4.13 ist daher für die unterschiedlichen Auslage- 

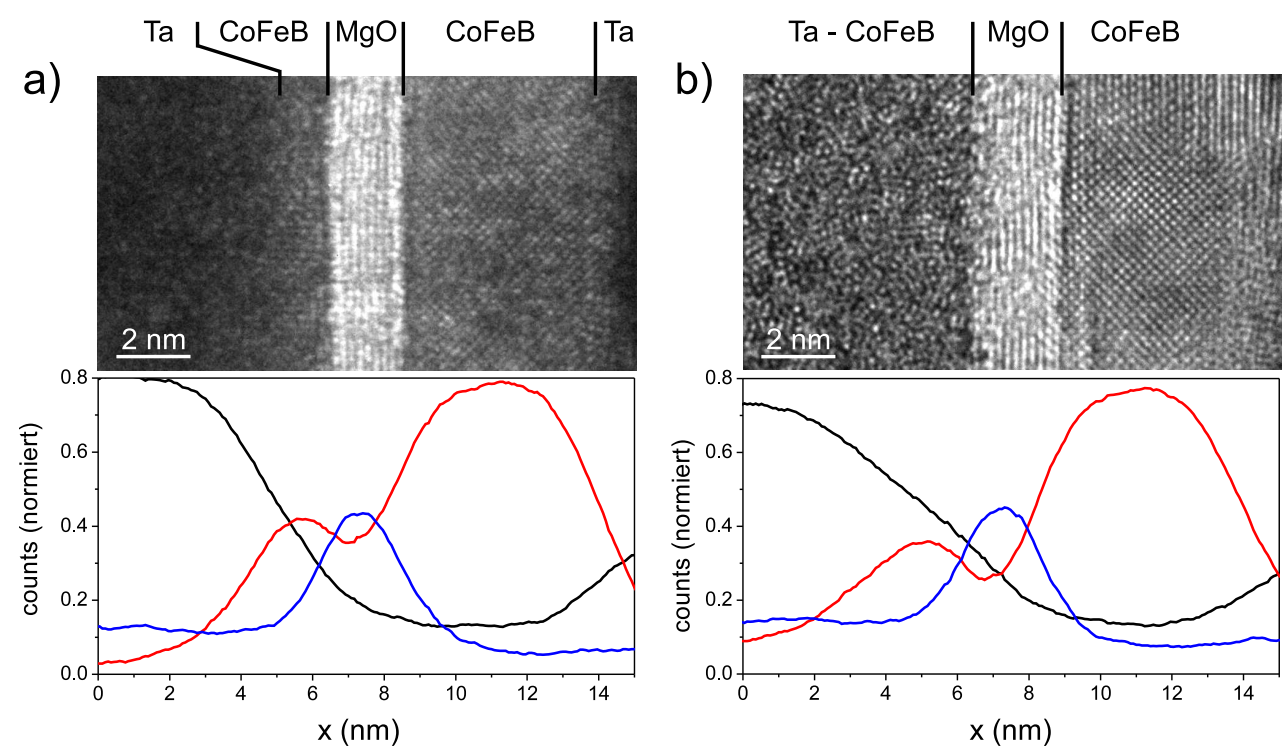

Abbildung 4.13: EDX-Linescans senkrecht zur Barriere für Ta (schwarz), CoFe (rot) und $\mathrm{MgO}$ (blau). Darüber zugehörige HRTEM-Abbildungen der Pseudo-Spinvalves nach Auslagerung bei $400^{\circ} \mathrm{C}$ (a) und $500^{\circ} \mathrm{C}$ (b) in gleicher Breite.

rungstemperaturen ein direkter Vergleich von EDX-Linescans senkrecht zur Barriere zusammen mit einem entsprechenden Ausschnitt aus der HRTEM-Abbildung gezeigt. Für die Darstellung der Kurven in den EDX-Scans wurden jeweils die Signale von Magnesium und Sauerstoff sowie die für Kobalt und Eisen zusammengefasst und alle Signale vom Hintergrund befreit. Zur Reduzierung des Rauschens ist der gleitende Mittelwert über 25 Messpunkte dargestellt. Bei einen Messpunktabstand von 0,08 $\mathrm{nm}$ ergibt sich damit eine Auflösung von $2 \mathrm{~nm}$, was in etwa auch dem Bereich entspricht, der bei einem EDX-Scan zur Signalentstehung beiträgt. Für die Auftragung wurden die jeweiligen Counts auf die Summe der Counts aller dargestellten Elemente zuzüglich der Counts für Ruthenium, welches aber nicht in der Auftragung gezeigt ist, normiert.

Es sind deutliche Unterschiede in der Tantalverteilung zu erkennen. So liegt die relative Anzahl der Counts für Tantal im Fall der bei $500^{\circ} \mathrm{C}$ ausgelagerten Probe immer über der relativen Anzahl der Counts für Kobalt-Eisen der oberen Elektrode. Für die bei $400^{\circ} \mathrm{C}$ ausgelagerte Probe gibt es hingegen einen Bereich oberhalb der Barriere in dem die Kobalt-Eisen Kurve das relative Maximum an Counts zeigt. Da die nominelle Schichtdicke der oberen CoFeB-Elektrode mit 1,5 nm be- 

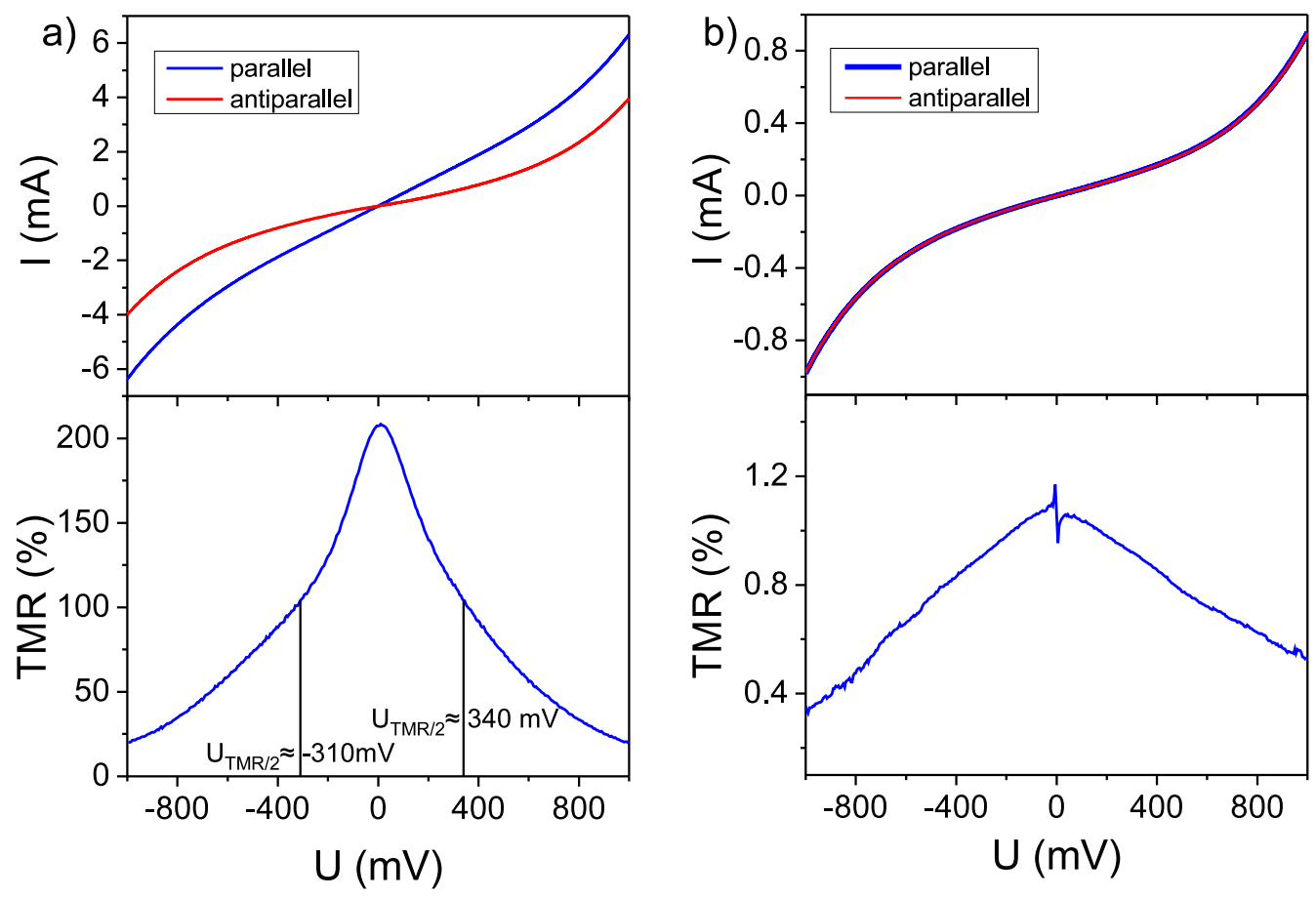

Abbildung 4.14: Tunnelkennlinien für parallele und antiparallele Ausrichtung der Elektroden und daraus berechneter TMR der PSVs nach Auslagerung bei $400^{\circ} \mathrm{C}$ (a) und $500^{\circ} \mathrm{C}(\mathrm{b})$.

reits am Auflösungslimit des EDX liegt, kann aus den Linescans die exakte Dicke der Schichten und die Position der Grenzflächen nicht bestimmt werden. Dennoch zeigt der Vergleich der beiden Proben, dass das Tantal der $500^{\circ} \mathrm{C}$ Probe näher an die Barriere heranreicht und der flachere Anstieg der Tantalkurve lässt auf einen gleitenden Konzentrationsanstieg statt einer scharfen Grenzfläche zwischen Tantal und Kobalt-Eisen schließen. Das Tantal scheint bis an die Grenzfläche zur Barriere diffundiert zu sein und so die bcc-Kristallisation des CoFeB zu verhindern.

In Abbildung 4.14 sind die Tunnelkennlinen der beiden PSV-Systeme und der daraus berechnete spannungsabhängige Tunnelmagnetowiderstand aufgetragen. Die Kennlinie der $400^{\circ} \mathrm{C}$-Probe zeigt in paralleler Konfiguration das typische Verhalten für kohärentes Tunneln in Systemen mit MgO-Barriere, welches 

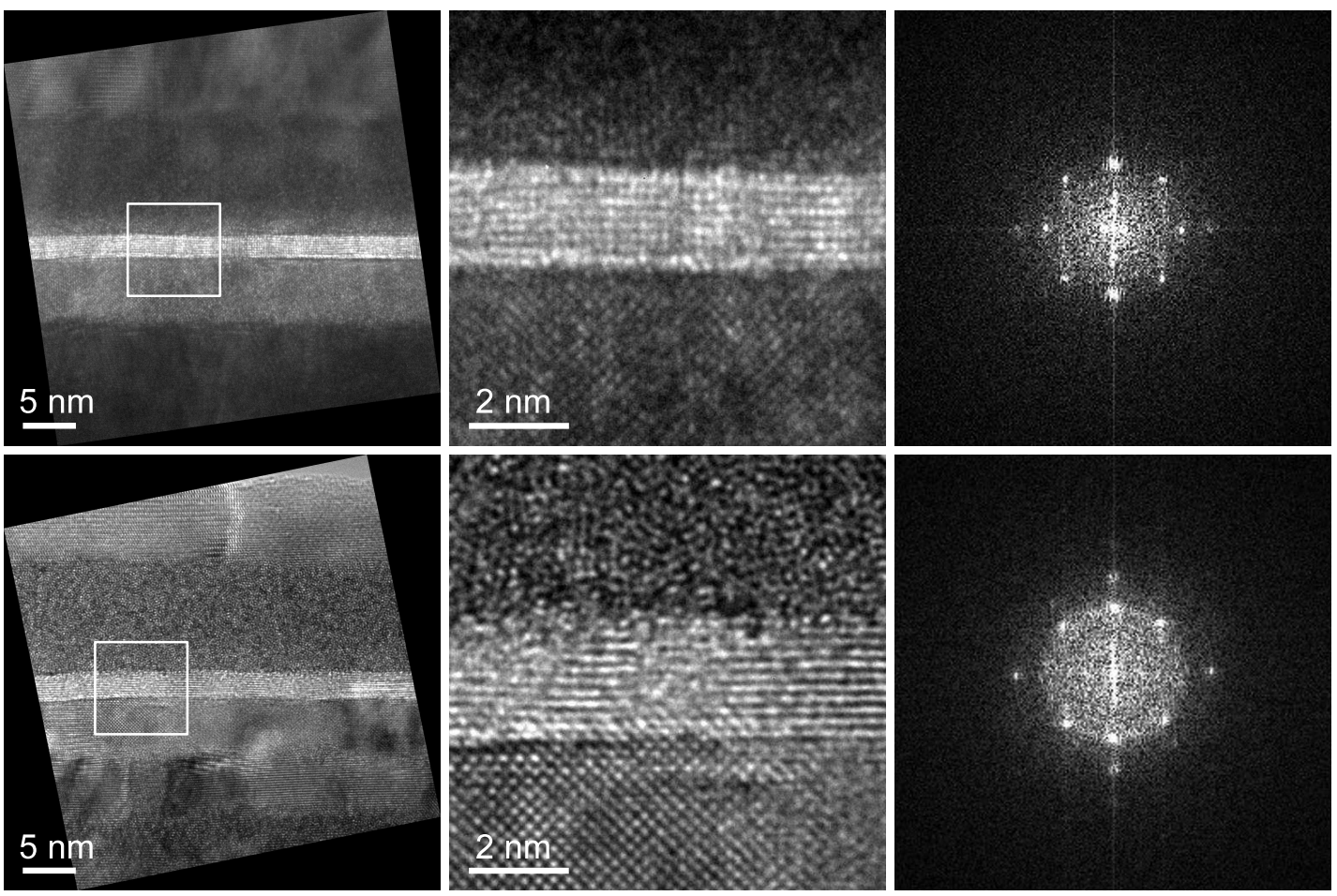

Abbildung 4.15: Vergleich der PSV-Systeme mit Auslagerungstemperaturen von $400^{\circ} \mathrm{C}$ (oben) und $500^{\circ} \mathrm{C}$ (unten). Rechts ist eine Fouriertransformation der vergrößerten Ausschnitte (Mitte) der HRTEM-Abbildungen (links) dargestellt.

sich in einem ausgeprägen, fast linearen Bereich um Null äußert. Die Kennlinie für die antiparallele Konfiguration zeigt dieses Verhalten nicht, da hier der effektive Tunnelkanal der $\Delta_{1}$-Zustände nicht zur Verfügung steht. Dies spiegelt sich auch im spannungsabhängigen Verhalten des TMR wider. Die Abnahme des TMR mit zunehmender Spannung ist hier im Wesentlichen auf die stärkere Nichtlinearität des Tunnelstromes in antiparalleler Konfiguration zurückzuführen, wird aber auch in anderen Systemen ohne kohärentes Tunneln beobachtet. Häufig wird die Spannung, bei welcher der TMR auf die Hälfte seines Maximalwertes abgefallen ist, als Maß für die Qualität von Tunnelelementen herangezogen. Diese TMR-Halbwertsspannung ist hier eingezeichnet und beträgt auf der negativen Seite (Elektronenflussrichtung von der oberen zur unteren Elektrode) $U_{\mathrm{TMR} / 2} \approx-310 \mathrm{mV}$ und auf der positiven Seite $U_{\mathrm{TMR} / 2} \approx 340 \mathrm{mV}$. Der TMR zeigt also nur wenig Asymmetrie, wobei noch auffällt, dass der Abfall für Spannungen größer als $U_{\mathrm{TMR} / 2}$ auf der negativen Seite etwas flacher verläuft. 
Die Kennlinien der $500^{\circ} \mathrm{C}$-Probe liegen nahezu übereinander und zeigen den Verlauf wie im antiparallelen Zustand der $400^{\circ}$ C-Probe. Der Symmetrie-FilterEffekt der MgO-Barriere zeigt hier keine Auswirkung mehr, vermutlich deshalb, weil die passenden Zustände in der oberen Elektrode nicht existieren. Es ist noch ein geringer Magnetowiderstand messbar, der aber kein TMR im Sinne von scharf schaltenden Zuständen ist. Wie in Abbildung 4.12 c) zu erkennen ist, wird zumindest für Magnetfelder bis $30 \mathrm{mT}$ gar kein paralleler Zustand mit dann konstant bleibendem Widerstand erreicht. Dennoch zeigt dieser Magnetowiderstand auch ein spannungsabhängiges Verhalten mit deutlicher Asymmetrie. Der Abfall ist für die Elektronenflussrichtung von der intakten unteren Elektrode in die zerstörte obere geringer als für die entgegengesetzte Richtung.

Ein weiterer Vergleich der beiden PSV-Systeme ist in Abbildung 4.15 gezeigt. Auf der linken Seite sind jeweils HRTEM-Aufnahmen des gesamten Schichtstapels zu sehen, in denen die Bereiche weiß markiert sind, auf welchen die rechts dargestellte Fouriertransformation ausgeführt wurde, sind in der Mitte vergrößert gezeigt. In beiden Fällen zeigt die Fouriertransformation Reflexe, die sich kristallinem $\mathrm{MgO}$ bzw. CoFe zuordnen lassen. Eine Zuordnung der Reflexe für beide möglichen Durchstrahlrichtungen mit kristallinem Kontrast findet sich in Abbildung 4.19. Darüberhinaus lässt sich in der Fouriertransformation der $500^{\circ} \mathrm{C}$ Probe ein ringförmiger Kontrast erkennen, welcher die amorphe bzw. nanokristalline Struktur der tantaldurchsetzten oberen CoFeB-Elektrode widerspiegelt.

\subsubsection{Vollkristallines Pseudo-Spinvalve}

In diesem Abschnitt wird ein weiteres Pseudo-Spinvalve mit dem Schichtaufbau $\mathrm{SiO}_{x} / 20 \mathrm{~nm} \mathrm{Ta} / 5,4 \mathrm{~nm} \mathrm{CoFeB} / 2,3 \mathrm{~nm} \mathrm{MgO} / 2,5 \mathrm{~nm} \mathrm{CoFeB} / 25 \mathrm{~nm} \mathrm{Ta} / 30 \mathrm{~nm} \mathrm{Ru}$ und einer anschließenden Auslagerung bei $450^{\circ} \mathrm{C}$ für eine Stunde behandelt. Es unterscheidet sich von den Vorherigen dadurch, dass es in einem Ultrahochvakuumsystem mit besserem Basisdruck hergestellt wurde. Außerdem wurden mittels Elektronenstrahllithographie und Sputterätzen elliptische Elemente mit den Achsenlängen $1 \mu \mathrm{m}$ und $4 \mu \mathrm{m}$ erzeugt. Die Elemente zeigen einen Flächenwiderstand von 1-2 M $\Omega \mu^{2}$ und einen TMR von bis zu $310 \%$.

In der HRTEM-Abbildung auf der rechten Seite von Abbildung 4.16 sind zwei Bereiche mit einer deutlichen (001)- bzw. (011)-Orientierung des MgO in Durchstrahlrichtung markiert, die deutlich pseudomorphes Wachstum der angrenzenden CoFeB-Elektroden zeigen. Die in Abbildung 4.16 oben gezeigten Mittelungen sind jeweils über einen 10 Streifen breiten Teilbereich dieser markierten Zonen ausgeführt worden. In beiden Fällen zeigt sich eine Reichweite der induzierten Ordnung bis weit in die CoFeB-Elektroden hinein. Der Vergleich mit den nominellen Dicken dieser Schichten lässt darauf schließen, dass diese in weiten Bereichen vollständig 

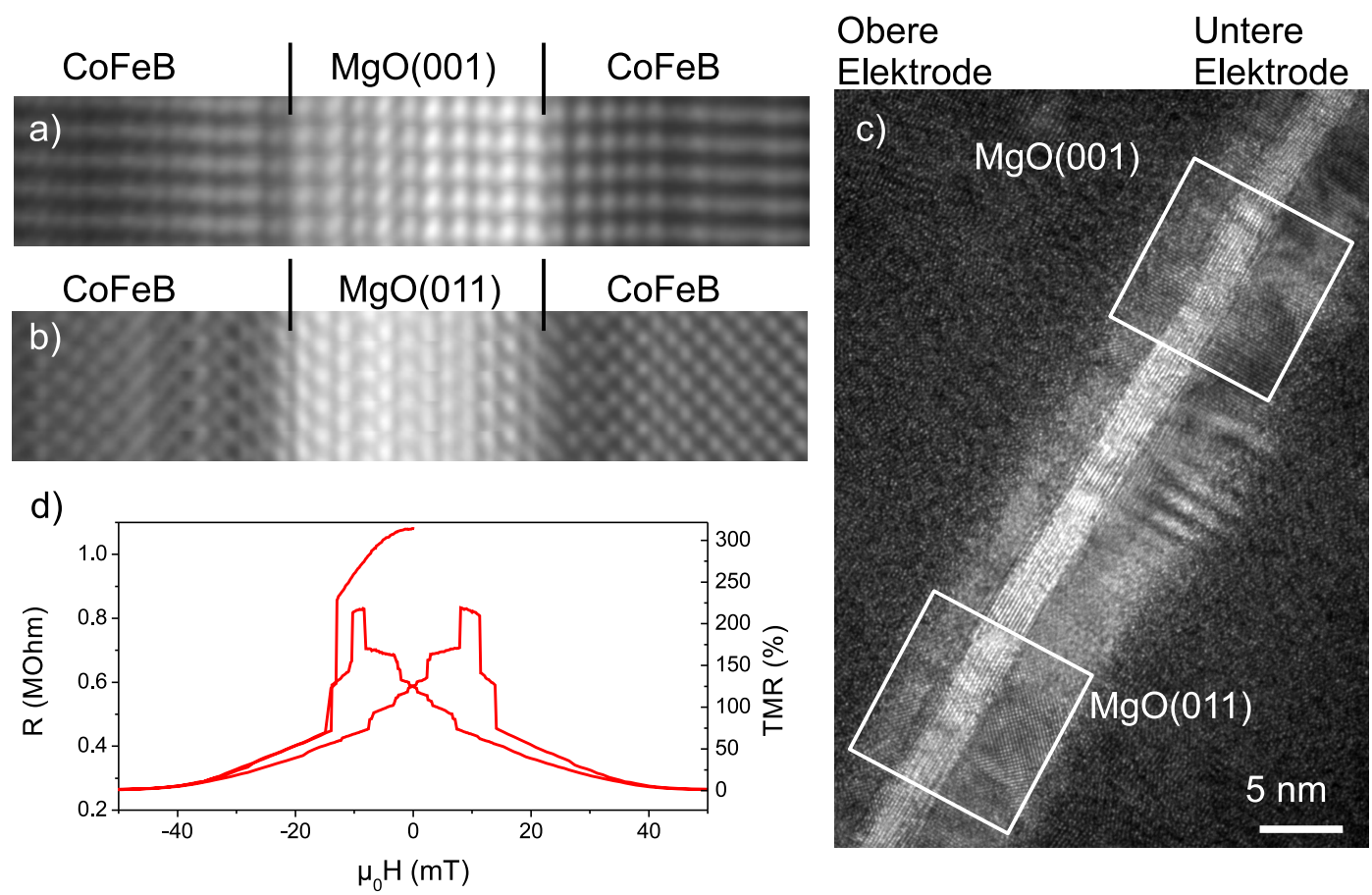

Abbildung 4.16: HRTEM Aufnahme (c) eines optimierten Pseudo-Spinvalve Systems mit 310\% TMR und die Ergebnisse der Mittelungen (a,b) über zwei Bereiche der Barriere mit je 10 Streifen Breite. Die unterschiedlichen Orientierungen des MgOs sind auch in den Mittelungen erkennbar. Zugehörige Magnetowiderstandsmessung (d).

durchkristallisiert sind.

In Abbildung 4.17 sind den gemittelten experimentellen Abbildungen für die beiden Kristallrichtungen (001) bzw. (011) simulierte Abbildungen gegenübergestellt, für die jeweils eine Superzelle angepasst wurde. Dabei wurde für den Barrierenbereich die Gitterkonstante eines MgO-Volumenkristalls verwendet. Im Bereich der CoFe-Elektroden wurden in den Ebenen parallel zur Grenzfläche die Abstände vom $\mathrm{MgO}$ übernommen, die Abstände der Ebenen senkrecht zur Barriere wurden variiert, um bestmögliche Übereinstimmung mit den experimentellen Abbildungen zu erreichen. Für den Barrierenbereich, der in Durchstrahlrichtung eine $\mathrm{MgO}(011)$-Orientierung zeigt, wurde mit einem Lagenabstand von 1,45 A eine sehr gute Übereinstimmung erzielt. Dieser Wert ist fast identisch mit der halben Gitterkonstante von Eisen, die 2,86 A beträgt. Der andere Barrierenbereich zeigt 
a)

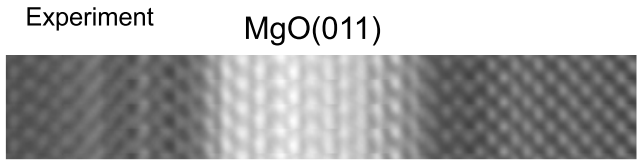

Simulation
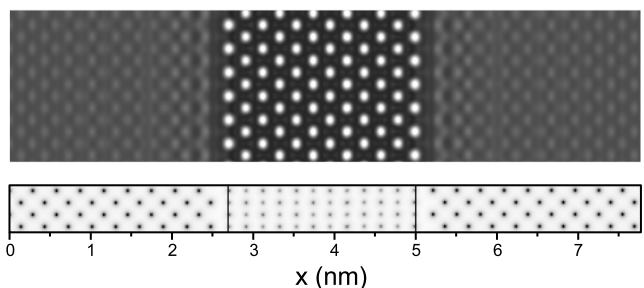

b)

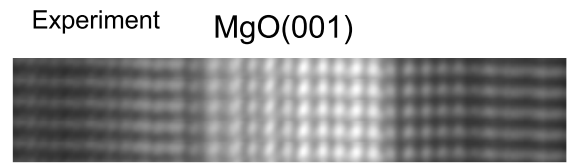

Simulation
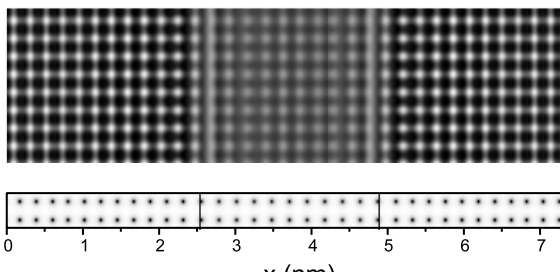

Abbildung 4.17: Vergleich von gemittelten experimentellen Abbildungen mit simulierten Abbildungen für ein weitgehend kristallisiertes Pseudo-Spinvalve. Für die Simulationen wurde eine vollständig kristalline Superzelle verwendet.

eine $\mathrm{MgO}(001)$-Orientierung in Durchstrahlrichtung, hier konnte eine zufriedenstellende Angleichung der Atomlagenabstände im Eisen erst mit einem Abstand von 1,95 $\AA$ erreicht werden. Der Grund für diese Abweichung ist unklar, möglicherweise variiert aber auch die Gitterkonstante des $\mathrm{MgO}$ entlang der Barriere aufgrund unterschiedlicher Verspannungen durch die Gitterfehlpassung zwischen Eisen und $\mathrm{MgO}$. In diesem Fall wäre die Annahme von unverspanntem $\mathrm{MgO}$ als Barriere falsch und die Atomabstände in der Superzelle wären insgesamt zu großs. Am Übergang von $\mathrm{MgO}$ zu Eisen zeigt sich weder in der experimentellen noch in der simulierten Abbildung ein Kontrastsprung entlang der Atomreihen. Da für die Simulation eine kristalline Superzelle verwendet wurde, ist dies konsistent mit den Beobachtungen in Abschnitt 4.1.3. Aus dem Fehlen des Kontrastsprungs in der experimentellen Abbildung kann hier auf mehrere kristalline Lagen Kobalt-Eisen an der Grenzfläche geschlossen werden.

Entlang der Barriere in Abbildung 4.16 sind auch zwischen den markierten Bereichen deutlich die kristallinen Lagen der MgO-Barriere zu erkennen. Diese Lagenstruktur setzt sich in unterschiedlich starker Ausprägung auch in die CoFeB-Schichten fort, so dass auch in diesen Bereichen ein pseudomorphes Wachstum vorliegen kann. Eine solche kollumnare Struktur des gesamten Elements mit Säulen von jeweils ca. $10 \mathrm{~nm}$ Durchmesser und pseudomorphem Schichtaufbau senkrecht zur Barriere, wie sie in Abbildung 4.18 dargestellt ist, wurde auch von CHOI ET. AL ${ }^{60]}$ gefunden. Jede dieser Säulen ist epitaktisch aufgebaut, die (100)-Richtungen von Eisen und MgO liegen parallel und in Wachstumsrichtung 


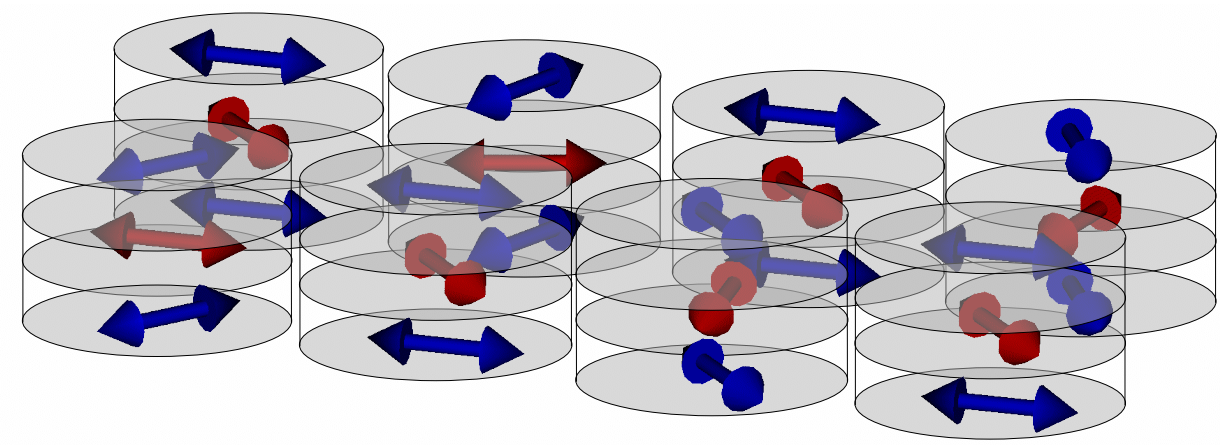

Abbildung 4.18: Schematische Darstellung eines magnetischen Tunnelelements bestehend aus einer Gruppe von epitaktisch aufgebauten Säulen, die aber gegeneinander verdreht sind. Jede Säule für sich erfüllt die Bedingungen für kohärentes Tunneln. Modell nach CHOI ET AL. 60.

senkrecht zur Barriere und in der Grenzflächenebene liegt die (011)-Richtung des Eisens parallel zur (001)-Richtung des MgO. Innerhalb dieser Säulen sind die Bedingungen für kohärentes Tunneln erfüllt, aber an den Korngrenzen im MgO könnte man Leckströme erwarten, die den Magnetowiderstand reduzieren. Bei einem Vergleich solcher Tunnelelemente mit texturierten MgO-Barrieren mit solchen, die vollständig epitaktisch gewachsen wurden, fanden mehrere Gruppen 11114161, dass sich die Flächenwiderstände in den Systemen kaum unterscheiden. MizUGUCHI ET AL. ${ }^{62}$ untersuchten die Eigenschaften der Korngrenzen direkt mit Rastertunnelspektroskopie. Dabei fanden sie in der Topographie an den Korngrenzen lediglich Absenkungen von 0,2 nm, was einer Monolage MgO entspricht. Die Tunnelkennlinien aufgenommen an einer Korngrenze und in einer Kornmitte zeigten fast identisches Verhalten, was bedeutet, dass der Tunnelstrom homogen durch die gesamte Barriere fließst.

In Abbildung 4.19 sind auf der linken Seite die in Abbildung 4.16 markierten Bereiche vergrößert dargestellt. Die Insets zeigen jeweils eine Fouriertransformation des weiß umrandeten Ausschnitts, um die Reflexe des CoFeB isolieren zu können. In der Mitte sind die Fouriertransformationen der gesamten Bereiche zu sehen, die auch die Reflexe enthalten, die dem $\mathrm{MgO}$ zuzuordnen sind. Für sämtliche auftretenden Reflexe konnte eine Zuordnung entweder zum MgO oder zum in bcc-Struktur kristallisierten Kobalt-Eisen gefunden werden, wie sie auf der rechten Seite bezeichnet sind. Es wird auch hier deutlich, dass in beiden Bereichen pseudomorphes Wachstum über den gesamten Ausschnitt vorliegt. 


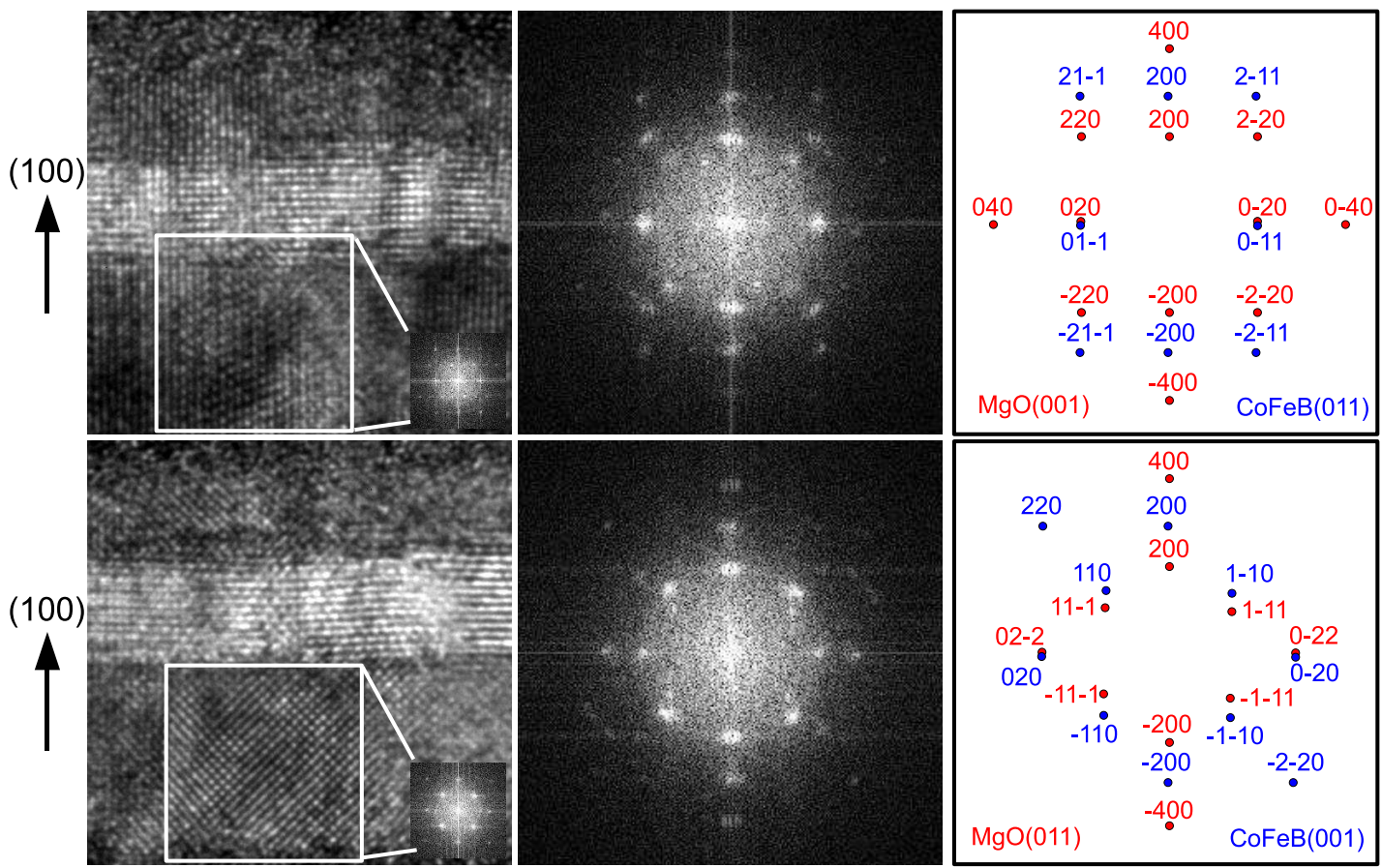

Abbildung 4.19: Fouriertransformationen (Mitte) der HRTEM-Abbildungen (Links) für verschiedene Bereiche der Barriere. Die Insets in den HRTEM-Abbildungen zeigen Fouriertransformatinen über die markierten Teilbereiche der CoFeB-Elektroden, um deren Reflexe separieren zu können. Rechts erfolgt die Zuordnung der auftretenden Reflexe.

Größere noch amorphe Bereiche lassen sich aus den Fouriertransformationen der HRTEM-Abbildungen nicht ableiten. Die auftretenden Reflexe lassen sich so zuordnen, dass in der oberen Abbildung nur MgO-Reflexe auftauchen, die zu einer Durchstrahlung in (001)-Richtung passen und nur solche für bcc-Eisen, die zu einer Durchstrahlung in (011)-Richtung passen, wie es für epitaktisches Wachstum mit der Wachstumsrichtung (100) zu erwarten ist. Für die untere Abbildung erfolgt die Zuordnung umgekehrt zur Durchstrahlung in $\mathrm{MgO}(011)$-Richtung und $\mathrm{CoFe}(001)$-Richtung. Hier tauchen aber noch zwei weitere Reflexe auf, die nicht zu dieser Zuordnung passen. Sie lassen sich aber den MgO-Reflexen (020) und (0-20) zuordnen und stammen vermutlich von dem Barrierenbereich ganz links in der HRTEM-Abbildung, der aber schon außerhalb der epitaktischen Säule liegt.

In Abbildung 4.20 sind die Tunnelkennlinien und der daraus berechnete spannungsabhängige TMR aufgetragen. In der Kennlinie für die parallele Konfigu- 

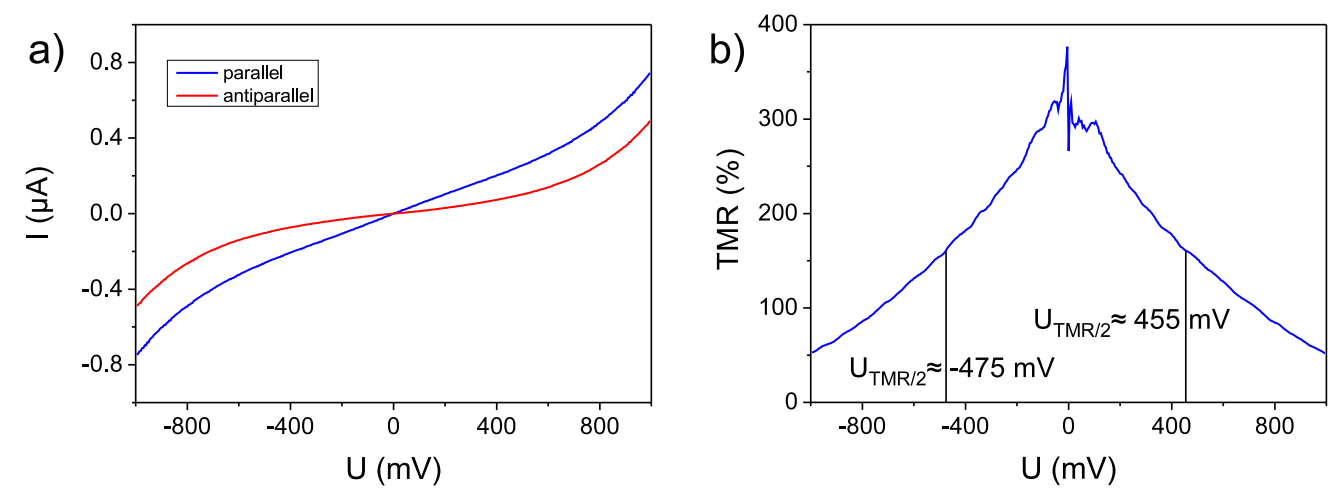

Abbildung 4.20: Tunnelkennlinien für parallele und antiparallele Ausrichtung der Elektroden (a) und daraus berechneter TMR (b) des vollkristallinen Pseudo-Spinvalves.

ration ist auch hier der ausgeprägte lineare Bereich um Null auszumachen. Die Kennlinie für den antiparallelen Fall zeigt eine wesentlich stärkere Nichtlinearität und führt auch hier zu einem abnehmenden TMR mit zunehmender Spannung, mit noch geringerer Asymmetrie als bei dem oben gezeigten teilkristallinen PSV. In den deutlich gestiegenen TMR-Halbwertsspannungen zeigt sich eine hohe Qualität dieses Tunnelelements, was im Vergleich mit den Strukturuntersuchungen auf den hohen Kristallisationsgrad der Elektroden zurückzuführen ist. Die Sprünge in der Kurve nahe Null sind in dem großen Widerstand des mikrostrukturierten Elements von fast $1 \mathrm{M} \Omega$ im Zusammenhang mit der Art der Messung begründet. Bei zu kleinen Spannungen sinkt der Strom auf Werte nahe der Auflösungsgrenze des Messgeräts und es kommt zu einer zu groben Quantisierung des Strommesswertes.

\subsection{Dielektrischer Durchbruch magnetischer Tunnelelemente}

Im Folgenden werden die Auswirkungen von elektrischer Überlastung auf magnetische Tunnelelemente in Abhängigkeit von ihrer Strukturgröße verglichen. Dabei handelt es sich zum einen um ein großflächiges quadratisches Element mit einer Kantenlänge von 22,5 $\mathrm{mm}$ und zum anderen um ein mittels Elektronenstrahllithographie nanostrukturiertes elliptisches Element mit einer Größe von $360 \mathrm{~nm}$ $\times 150 \mathrm{~nm}$ und ultradünner Barriere. Die Ergebnisse wurden in den Artikeln von

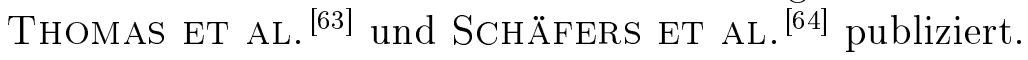




\subsection{1 Überspannungszerstörte MTJs}

Bei den in diesem Teil vorgestellten Proben, welche von der Arbeitsgruppe Thin Films and Physics of Nanostructures von Andy Thomas aus Bielefeld zur Verfügung gestellt wurden, handelt es sich um ein magnetisches Tunnelsystem mit einer zusätzlichen antiferromagnetischen Schicht, welche die Magnetisierung der unteren ferromagnetischen Elektrode durch Austauschkopplung festlegt. Dies hat den Vorteil, dass die magnetischen Schichten besser separieren, bringt aber auch den Nachteil, dass das Mangan aus dem hier verwendeteten Antiferromagneten $\mathrm{Mn}_{83} \mathrm{Ir}_{17}$ in andere Schichten diffundieren kann und den TMR auf diese Weise negativ beeinflusst. Der Schichtstapel auf oxidiertem Silizium hat die Abfolge $5 \mathrm{~nm} \mathrm{Ta} / 30 \mathrm{~nm} \mathrm{Cu} / 5 \mathrm{~nm} \mathrm{Ta} / 5 \mathrm{~nm} \mathrm{Cu} / 12 \mathrm{~nm} \mathrm{MnIr} / 4 \mathrm{~nm} \mathrm{CoFeB} / 0,5 \mathrm{~nm} \mathrm{Mg} / 1,5 \mathrm{~nm}$ $\mathrm{MgO} / 6 \mathrm{~nm} \mathrm{CoFeB} / 5 \mathrm{~nm} \mathrm{Ta} / 40 \mathrm{~nm} \mathrm{Cu} / 30 \mathrm{~nm} \mathrm{Au}$ und wurde zur Ausrichtung des Antiferromagneten eine Stunde bei $350^{\circ} \mathrm{C}$ in einem Magnetfeld von 6500 Oe ausgelagert. Anschließend wurden mittels optischer Lithographie und Sputterätzen die quadratischen Elemente mit einer Kantenlänge von 22,5 $\mu \mathrm{m}$ herauspräpariert.

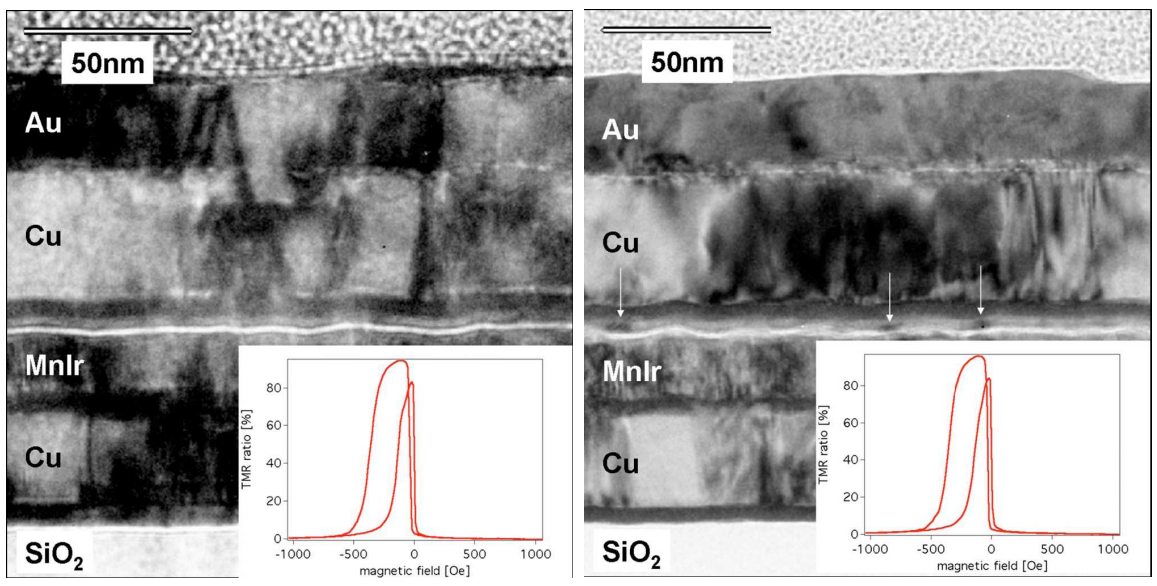

Abbildung 4.21: Übersichts-TEM-Aufnahmen eines intakten Tunnelelementes (links) und eines elektrisch überlasteten (rechts). Die Einschübe zeigen die zugehörigen Magnetowiderstandskurven vor der elektrischen Überlastung.

Nach der Durchführung von Standardtransportmessungen wurde die Hälfte der Elemente einer starken Belastung durch Anlegen einer Spannung von 1,5 V ausgesetzt. Abbildung 4.21 zeigt auf der linken Seite ein intaktes Element vor der elektrischen Überlastung. Die eingeschobene Magnetowiderstandskurve desselben Elements liefert einen Magnetowiderstand von 95\%. Man erkennt deutlich die 
oben beschriebene Schichtabfolge, und die Barriere wirkt schon in dieser Vergrößerung sehr homogen.

Auch in der zugehörigen HRTEM Aufnahme in Abbildung 4.22 links ist die homogen kristalline $\mathrm{MgO}$ Barriere zwischen den CoFeB-Elektroden zu sehen, die überwiegend den typischen Kontrast eines amorphen Materials aufweisen. Es gibt keine Anzeichen für Barrierendurchbrüche.

Dies ändert sich, wenn die Elemente einer hohen Spannungsbelastung ausgesetzt werden, der zu elektrischen Durchbrüchen der Barriere führt. Schon in der Übersichtsaufnahme in Abbildung 4.21 rechts sind die Veränderungen deutlich. Der Einschub zeigt wieder die Magnetowiderstandskurve dieses Elements vor dem Anlegen einer Spannung von 1,5 V, welche die Vergleichbarkeit mit der Referenzprobe verdeutlicht. Weiße Pfeile markieren die Bereiche, in denen ein deutlicher Beugungskontrast in der oberen CoFeB-Elektrode sichtbar wird, was darauf hindeutet, dass das Material hier zumindest teilweise kristallisiert ist. Unterhalb solcher Bereiche scheint die Barriere durchbrochen zu sein.
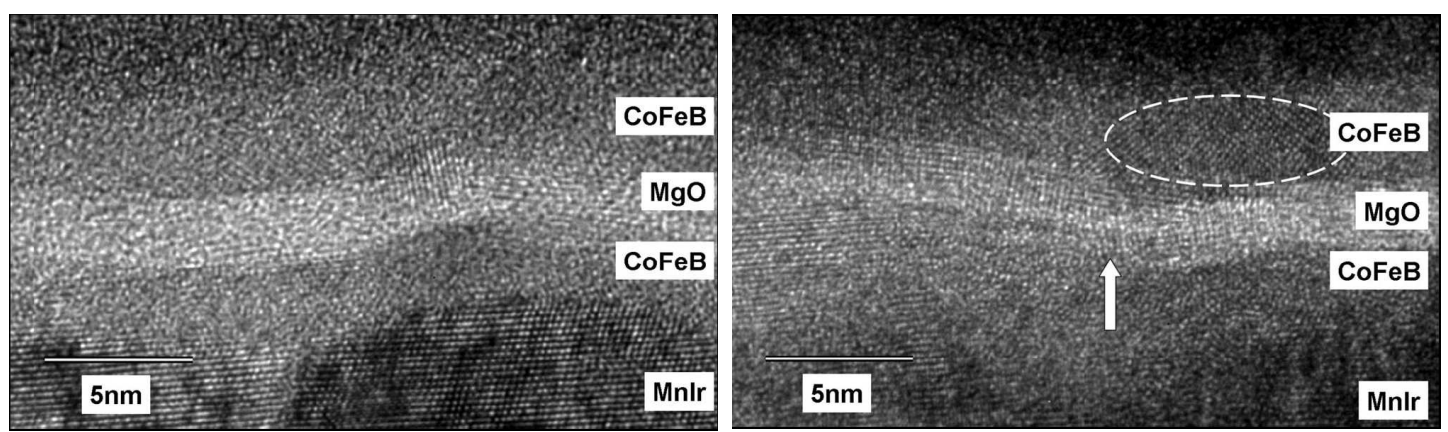

Abbildung 4.22: HRTEM-Aufnahem eines intakten Tunnelelementes (links) und eines elektrisch überlasteten (rechts).

Eine hochaufgelöste TEM-Aufnahme eines dieser dielektrischen Durchbrüche, ein sogenanntes Pinhole, ist in Abbildung 4.22 auf der rechten Seite gezeigt. In der Mitte ist der Bruch der MgO-Barriere, markiert mit einem weißen Pfeil, auszumachen. Oberhalb des dielektrischen Durchbruchs ist ein Korn kristallinen KobaltEisens markiert, das offenbar durch lokales Annealing in Folge des in dieser Region erhöhten Stromes nach dem Durchbruch entstanden ist.

Eine Beschreibung des dielektrischen Durchbruchs isolierender dünner Schichten wird durch das von MCPHERSON UND MoguL ${ }^{65}$ vorgestellte E-Modell für $\mathrm{SiO}_{2}$ gegeben. In diesem Modell, das von Thomas ET AL. [3] auf MgO Barrieren angewendet wurde, ist das elektrische Feld und damit die Spannung über 
der Barriere der entscheidende Parameter, der zum initialen Durchbruch führt. Dies scheint auch hier erfüllt zu sein, da die Durchbruchspannung mit 1,5 V vergleichbar ist mit der in anderen Systemen, wie beispielsweise denjenigen mit $\mathrm{Al}_{2} \mathrm{O}_{3}$-Barrieren. Allerdings fließen durch die $\mathrm{MgO}$-Barrieren bei gleicher Dicke um den Faktor 200 größere Tunnelströme. Der Flächenwiderstand dieser MgOTunnelbarrieren beträgt $54 \mathrm{k} \Omega \mathrm{\mu m}^{2}$, während der von $\mathrm{Al}_{2} \mathrm{O}_{3}$-Barrieren gleicher Dicke etwa einen Wert von $10 \mathrm{M} \Omega \mu \mathrm{m}^{2}$ hat.

Dieser große Unterschied im Strom könnte das Auftreten vieler Pinholes in den MgO-Barrieren nach der Entstehung des initialen Durchbruchs erklären. Hierzu dient ein Modell von XI ET AL. ${ }^{66}$, welches besagt, dass anhaltende Strombelastung der Barriere zu einer konstanten Bildungsrate $n_{0}$ der Pinholes führt. Sofern die Pinholes nach ihrer Entstehung nicht weiter anwachsen, entwickelt sich der Flächenanteil $a_{p}$, welcher von den Pinholes bedeckt ist mit der Zeit nach:

$$
a_{p}(t)=1-\exp \left(-c n_{0} t\right)
$$

Hierbei ist $c$ eine Konstante. Unter der Annahme, dass die Bildungsrate der Pinholes $n_{0}$ proportional zum Strom $I$ ist, folgt direkt, dass $a_{p}^{M g O}(t)>a_{p}^{A l O_{x}(t)}$. Wenn man weiter annimmt, dass $n_{0} \propto I$ ist, bilden sich die Pinholes im MgO 200 mal schneller als in $\mathrm{AlO}_{x}$.

Veranschaulichen lässt sich dieses Verhalten, wenn man berücksichtigt, dass die Strombelastung der Barriere in der Umgebung eines Pinholes abnimmt, da das gut leitende Pinhole einen Großteil des Stromflusses übernimmt. Die Größe des Bereiches, der davon beeinflusst wird, hängt vor allem von dem Flächenwiderstand der Barriere ab. Dieser bestimmt damit auch den mittleren Abstand zweier benachbarter Pinholes, was auch für eine homogene Verteilung der Pinholes über die Barriere spricht. Ohne eine homogene Verteilung wäre es sehr unwahrscheinlich in einer einzigen TEM-Lamelle aus einem Element dieser Größe überhaupt einen Durchbruch zu finden. Die hier vorgestellte Probe zeigt aber gleich drei Pinholes auf $200 \mathrm{~nm}$. In TMR-Elementen mit $\mathrm{AlO}_{x}$-Barriere führt der hohe Flächenwiderstand hingegen dazu, dass der beeinflusste Bereich sehr viel größer ist. Deshalb bildet sich in solchen Elementen häufig nur ein einziger Durchbruch, welcher dann allenfalls noch anwächst.

\subsubsection{MTJs mit ultradünnen Barrieren}

Im Gegensatz zu den großflächigen Tunnelelementen, die im vorigen Abschnitt betrachtet wurden, handelt es sich im Folgenden um Elemente, welche im Hinblick auf Spinstrom induziertes Schalten (STT ${ }^{1}$ ) optimiert sind. Hierbei wird die

\footnotetext{
${ }^{1}$ engl.: spin-transfer torque
} 
Magnetisierung der freien ferromagnetischen Elektrode nicht durch ein angelegtes Magnetfeld geschaltet, sondern mit Hilfe eines Drehmoments, welches von der Spinpolarisation des Tunnelstromes selbst induziert wird. Da für ein solches Spinstrom induziertes Schalten sehr große Stromdichten benötigt werden, ist es erforderlich die Strukturgröße der Elemente zu verkleinern, um den Gesamtstrom klein zu halten und für ausreichende Wärmeabfuhr zu sorgen. Hierfür werden dünne Barrieren mit sehr kleinem Flächenwiderstand benötigt, um die nötigen Ströme mit nicht zu hohen Spannungen treiben zu können. Die Stabilität gegen elektrische Barrierendurchbrüche begrenzt also die erzielbaren Stromdichten und damit die Eignung der magnetischen Tunnelelemente für das Spinstrom induzierte Schalten. Mit den dünnen Barrieren nimmt man allerdings den Nachteil eines geringeren maximalen TMRs in Kauf, da nun auch die in der Barriere schneller abfallenden Minoritätszustände bei antiparalleler Ausrichtung der Magnetisierung eine höhere Tunnelwahrscheinlichkeit haben.
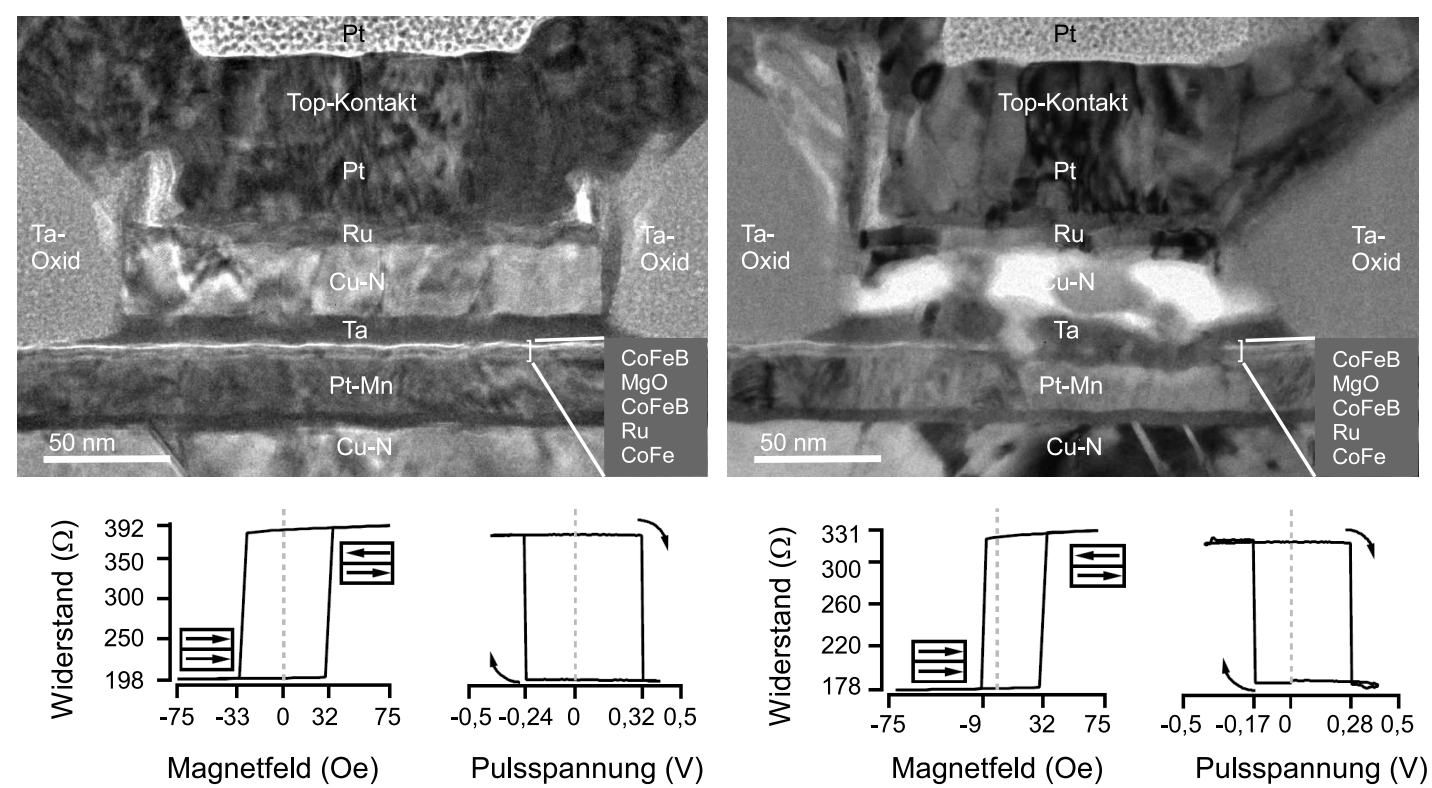

Abbildung 4.23: TEM-Aufnahmen eines intakten (links) und eines zerstörten MTJ. Darunter jeweils die zugehörigen Magnetowiderstandskurven $(\mathrm{R}(\mathrm{H})$, Minor Loops) und STT-Messungen $(\mathrm{R}(\mathrm{U}))$.

Die hier vorgestellten Proben stammen von der Firma Singulus, sie wurden in Bielefeld von der Arbeitsgruppe Andy Thomas strukturiert und elektrisch vermessen und haben den Schichtstapel $5 \mathrm{~nm} \mathrm{Ta} / 90 \mathrm{~nm} \mathrm{Cu}-\mathrm{N} / 5 \mathrm{~nm} \mathrm{Ta} / 20 \mathrm{~nm}$ 
$\mathrm{PtMn} / 2,2 \mathrm{~nm} \mathrm{Co} 70 \mathrm{Fe}_{30} / 0,8 \mathrm{~nm} \mathrm{Ru} / 2 \mathrm{~nm} \mathrm{CoFeB} / 1,1 \mathrm{~nm} \mathrm{MgO} / 1,5 \mathrm{~nm} \mathrm{CoFeB} / 10 \mathrm{~nm}$ $\mathrm{Ta} / 30 \mathrm{~nm} \mathrm{Cu}-\mathrm{N} / 7 \mathrm{~nm}$ Ru. Der gesamte Schichtstapel wurde in einem Magnetfeld von $1 \mathrm{~T}$ für 90 Minuten bei $360^{\circ} \mathrm{C}$ ausgelagert und anschließend mittels Elektronenstrahllithographie und Argon-Ionenätzen strukturiert. An den $360 \mathrm{~nm}$ $\times 150 \mathrm{~nm}$ großen elliptischen Elementen wurden Magnetowiderstandskurven (Minor-Loops) aufgenommen, sowie STT-Messungen mit $200 \mathrm{~ms}$ langen Schaltund Messpulsen durchgeführt. Die Widerstandsmessungen erfolgten bei einer Spannung von $10 \mathrm{mV}$ und die STT-Messungen ohne äußeres Magnetfeld. Ein Teil der Elemente wurde durch stetige Stromerhöhung zum elektrischen Durchbruch gebracht, wobei die Elektronenflussrichtung von der oberen freien in die untere gepinnte Elektrode war.

Abbildung 4.23 zeigt TEM-Aufnahmen eines Elements nach dielektrischem Durchbruch und eines intakten Referenzelements sowie die zugehörigen Magnetowiderstandskurven und STT-Messungen. Das intakte Element zeigt mit 99\% TMR einen typischen Wert für MTJs mit ultradünnen Barrieren. Die zugehörige STT-Messung liefert einen TMR von 93\%, was auf einen leicht reduzierten Widerstand in der antiparallelen Konfiguration zurückzuführen ist. Die Schaltspannungen von $-0,24 \mathrm{~V}$ und $0,32 \mathrm{~V}$ korrespondieren mit Schaltstromdichten von $-2,7 \times 10^{6} \mathrm{~A} / \mathrm{cm}^{2}$ bzw. $3,8 \times 10^{6} \mathrm{~A} / \mathrm{cm}^{2}$. Das Element nach dielektrischem Durchbruch in Abbildung 4.23 rechts zeigt einen TMR von $86 \%$ bzw. $82 \%$ aus den STT-Messungen und Schaltstromdichten von $-2,2 \times 10^{6} \mathrm{~A} / \mathrm{cm}^{2}$ für das Schalten von paralleler zu antiparalleler Konfiguration bzw. $3,7 \times 10^{6} \mathrm{~A} / \mathrm{cm}^{2}$ in entgegengesetzter Richtung. Die TEM-Aufnahmen in Abbildung 4.23 zeigen den kompletten Schichtstapel mit Ausnahme der unteren Ta-Schicht, dabei bildet das Tantaloxid links und rechts der oberen Elektrode eine Isolierschicht, die während der Strukturierung eingebracht wird. Die sichtbare Kontaktbreite ist abhängig von der Position und Richtung, in der die TEM-Lamelle mit dem FIB aus dem elliptischen Element herausgeschnitten wurde und lässt daher keinen Rückschluss auf die genaue Größe des Elements zu. Unterhalb des Tantaloxids ist die MgO Tunnelbarriere als dünne weiße Linie zu erkennen.

Im Vergleich $\mathrm{zu}$ den vereinzelten Pinholes, die im vorigen Abschnitt für dicke Barrieren gezeigt wurden, treten hier beim Vergleich der TEM-Aufnahmen wesentlich drastischere Veränderungen auf. In den hellen Bereichen im oberen $\mathrm{Cu}-\mathrm{N}$ des zerstörten Elements ist das Material komplett verschwunden und es treten Hohlräume auf. Die MgO-Barriere im unteren Bereich des Bildes ist auf einer Breite von ca. $80 \mathrm{~nm}$ vollständig verschwunden. Diese Veränderungen sind noch deutlicher erkennbar, wenn man die STEM-Tomographie-Rekonstruktionen in Abbildung 4.24 betrachtet, welche in Zusammenarbeit mit K. Thiel am Fraunhofer Institut fuer Fertigungstechnik und Angewandte Materialforschung (IFAM) in Bremen angefertigt wurden. Hier sind Schnittbilder aus einer dreidimensiona- 

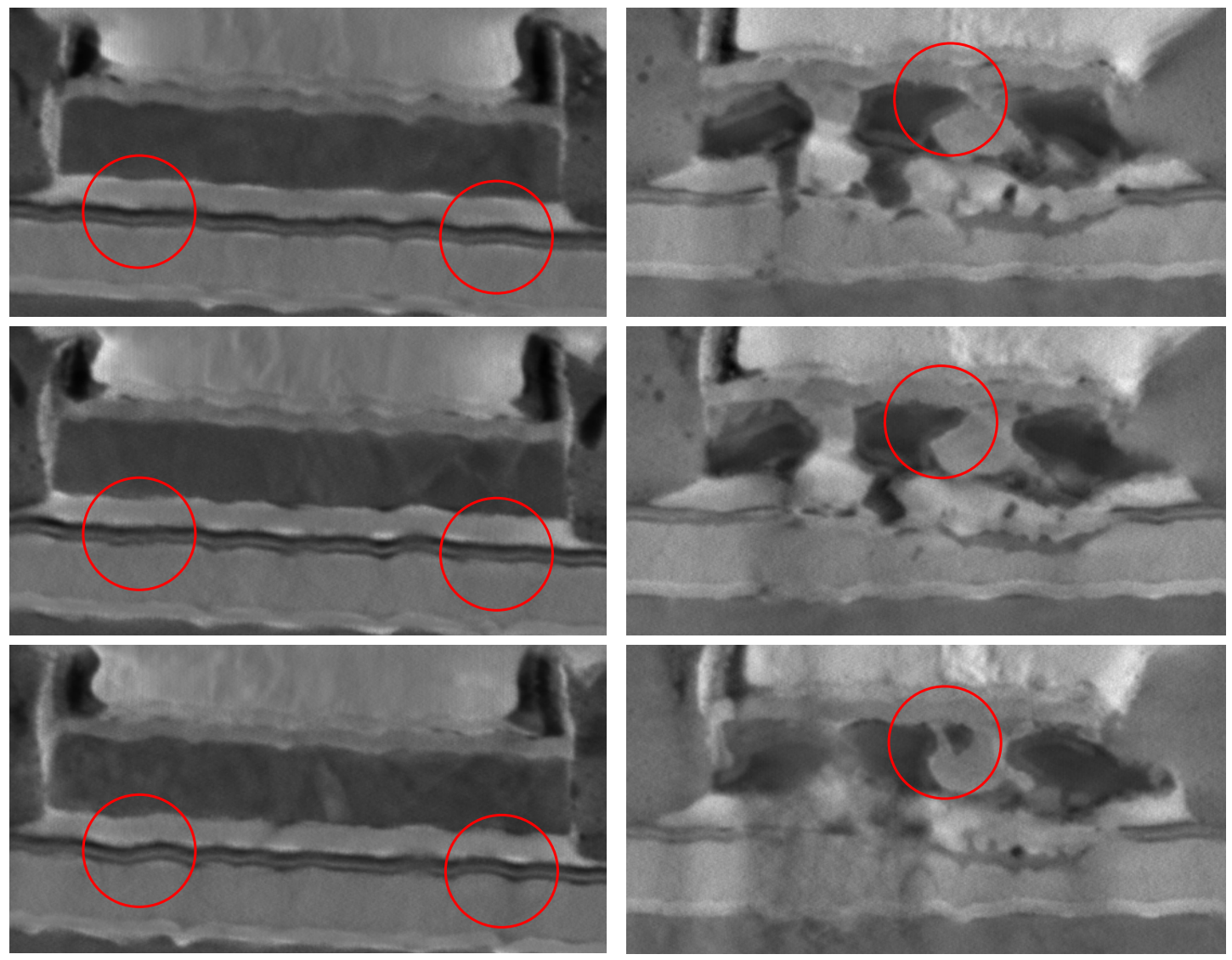

Abbildung 4.24: Schnittbilder für verschiedenen Tiefen aus einem STEM-Tomogramm für das intakte Element (links) und das zerstörte (rechts). Bereiche, in denen sich deutliche Änderungen in der Tiefe zeigen, sind rot markiert.

len tomographischen Rekonstruktion von STEM-Daten für verschiedene Tiefen der Elemente gegenübergestellt. Aus den Schnittbildern lässt sich zum Beispiel Information über die Grenzflächenrauigkeit in der Tiefe gewinnen. Für das intakte Element auf der linken Seite sind zwei Bereiche markiert, die eine deutliche Änderung im Grenzflächenverlauf zeigen. Diese Grenzflächenrauigkeit in Durchstrahlrichtung sind für den Vergleich gemittelter experimenteller und simulierter Abbildungen sehr störend, weil selbst in Bereichen, in denen die Grenzfläche in der Probenebene glatt erscheint, die Rauigkeit in die Tiefe die Ergebnisse verfälschen kann. Die rechte Seite in Abbildung 4.24 zeigt das zerstörte Element. Hier sind vor allem die durch den dielektrischen Durchbruch entstandenen Hohlräu- 
me interessant. In der Reihe ist eine Stelle markiert an der in einem Schnittbild Material vorhanden ist und in den anderen nicht. Es ist auch zu erkennen, dass die Hohlräume kompliziert geformt sind und in verschiedenen Tiefen Verbindung zueinander haben. Auch die Schichten der schweren Elemente Tantal und Ruthenium, die den stärksten Kontrast erzeugen, sind an vielen Stellen miteinander verbunden und bilden den elektrischen Kurzschluss.
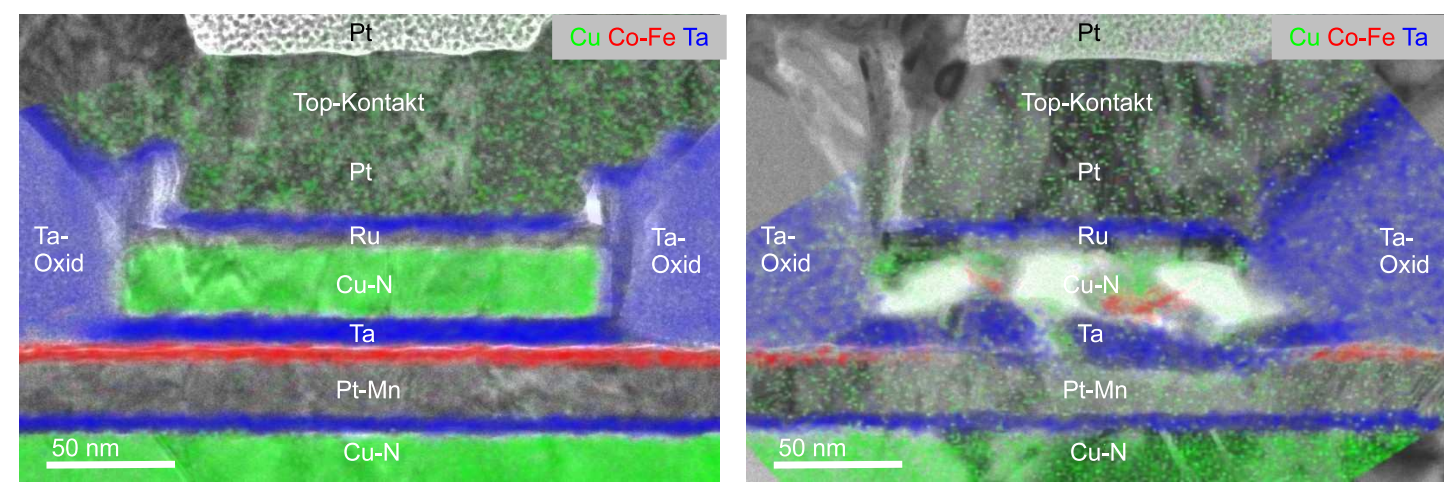

Abbildung 4.25: Farblich kodierte EDX-Scans als Overlay über den TEM-Aufnahmen des intakten (links) und zerstörten (rechts) Elements.

Die Tomographie-Rekonstruktionen in Abbildung 4.24 zeigen, dass ein ausgeprägter Materialtransport stattgefunden hat, welche Elemente dabei wie verschoben wurden zeigen sie aber nicht. Um dies aufzuklären, sind in Abbildung 4.25 farblich kodierte EDX-Karten über die TEM-Aufnahmen gelegt. Zur Wahrung der Übersichtlichkeit sind hier nur Kupfer, Kobalt-Eisen und Tantal dargestellt. Aufgrund der, schon in Abschnitt 4.2.2 erwähnten, begrenzten Auflösung des EDX von 1-2 nm, können die ferromagnetischen Elektroden nicht voneinander separiert werden. Es zeigt sich, dass nicht nur die Barriere betroffen ist, sondern insbesondere auch die ferromagnetischen Elektroden. Die Stromdichte betrug zum Zeitpunkt des Durchbruchs $7 \times 10^{6} \mathrm{~A} / \mathrm{cm}^{2}$ und $9 \times 10^{7} \mathrm{~A} / \mathrm{cm}^{2}$ danach. Diese hohen Stromdichten führten zu Elektromigration, welche elementspezifisch auf zwei Arten wirkt 67: Für Kobalt und Eisen, die weit oberhalb ihrer ursprünglichen Position wiederzufinden sind, ist der als direct force bezeichnete Mechanismus, der vom elektrischen Feld getrieben wird, dominierend, während der als wind force bezeichnete Mechanismus das Kupfer in Richtung des Elektronenstromes mitreißt.

Der Mechanismus des dielektrischen Durchbruchs unterscheidet sich bei diesen magnetischen Tunnelelementen mit ultradünnen Barrieren für hohe Stromdichten deutlich von dem im vorigen Abschnitt gezeigten. Möglicherweise ist auch hier der 
initiale Durchbruch spannungsgetrieben, jedoch sind die Stromdichten hier bis zu drei Größenordnungen höher. Diese Stromdichten führen zu starkem Materialtransport durch Elektromigration und der Durchbruch kann weiter anwachsen bis er sich über das gesamte Element erstreckt. 



\section{Zusammenfassung}

In dieser Arbeit wurde der Einfluss grenzflächennaher Ordnung in amorphen ferromagnetischen Elektroden auf die elektrischen Transporteigenschaften von magnetischen Tunnelelementen mit kristalliner MgO-Barriere untersucht. Dazu wurden mittels Focussed Ion Beam gezielt Querschnitte aus strukturierten magnetischen Tunnelelementen herauspräpariert, deren Transporteigenschaften vorher gemessen wurden, und mittels hochaufgelöster Transmissionselektronenmikroskopie untersucht. Der Vorteil dieses Vorgehens ist, dass die elektrischen Transportdaten und die TEM-Abbildungen von demselben Tunnelelement stammen und man keine Referenzproben für die verschiedenen Untersuchungsmethoden benötigt.

In Abschnitt 4.1 wurde die Grenzfläche zwischen kristallinem $\mathrm{MgO}$ und amorphem $\mathrm{CoFeB}$ zunächst anhand eines Modellsystems untersucht. Hierzu wurde ein CoFeB-Film auf ein kristallines MgO-Substrat gesputtert und anschließend bei unterschiedlichen Temperaturen ausgelagert. Querschnitte aus diesen Proben wurden im TEM untersucht, um Informationen über die Kristallisation des CoFeB bei den verschiedenen Auslagerungstemperaturen zu gewinnen. Für die Probe mit der niedrigsten Auslagerungstemperatur wurden HRTEM-Abbildungen entlang der Grenzfläche gemittelt und mit simulierten Abbildungen verglichen. Für die Simulation wurde ein Verfahren genutzt, bei dem für einen amorphen Festkörper eine zweidimensionale Verteilungsfunktion verwendet wird. Für die Konstruktion dieser zweidimensionalen Verteilungsfunktion des CoFeB wurde ein Modell entwickelt, das die grenzflächennahe Ordnung in den ferromagnetischen Elektroden mit nur einem Parameter beschreibt. Es zeigte sich, dass schon kleine Abweichungen von der kristallinen Ordnung zu charakteristischen Veränderungen in den HRTEM-Abbildungen führen. Es wurde auch versucht eine FeOZwischenschicht, die in der Literatur häufig für niedrige Magnetowiderstände verantwortlich gemacht wird, in das Modell für die Simulation zu integrieren. Die simulierten Abbildungen mit einer FeO-Zwischenschicht unterschieden sich nicht signifikant. Aufgrund der Vergleiche der simulierten Abbildungen mit gemittelten experimentellen Abbildungen, kann für eine Auslagerungstemperatur von $300^{\circ} \mathrm{C}$ eine kristalline Ordnung des $\mathrm{CoFeB}$ an der Grenzfläche von mehr als drei Monolagen ausgeschlossen werden.

In Abschnitt 4.2 wurden reale Tunnelelemente, in diesem Fall sogenannte Pseudo-Spinvalves, untersucht. Für eines der Elemente war nach sehr hoher 
Auslagerungstemperatur kein TMR mehr messbar. Dies konnte anhand der TEM-Abbildungen auf eine Ta-Diffusion in eine der ferromagnetischen Elektroden zurückgeführt werden. Ein weiteres Element derselben Probenreihe, aber mit niedrigerer Auslagerungstemperatur, zeigte einen TMR von 210\%. Da diese Elemente eine größere Grenzflächenrauigkeit aufweisen als das Modellsystem, ist es schwierig, Bereiche zu finden, in denen sich gemittelte Grenzflächenabbildungen über eine ausreichende Anzahl von Streifen erzeugen lassen, um sie mit simulierten Abbildungen zu vergleichen. Für dieses Element fand sich in den Elektroden ein ähnlich hoher Ordnungsgrad, wie in dem zuvor vorgestellten Modellsystem. Ein weiteres Element, das bei besseren Bedingungen hergestellt wurde, zeigte einen deutlich höheren TMR von $310 \%$. Hier wurden in beiden Elektroden kristalline Bereiche gefunden, deren Kristallorientierung, relativ zu der des angrenzenden MgOs, der eines pseudomorphen Wachstums entspricht, wie es für kohärentes Tunneln nötig ist. Dieses Element ist aus CoFeB/MgO/CoFeB-Säulen aufgebaut, die jede für sich pseudomorph gewachsen sind, deren Kristallrichtungen parallel zur Grenzfläche aber gegeneinander gedreht sind. Das pseudomorphe Wachstum geht dabei von einer texturierten MgO-Barriere aus und ist für den hohen TMR dieses Elements verantwortlich.

In Abschnitt 4.3 wurde der dielektrische Durchbruch von magnetischen Tunnelelementen anhand zweier unterschiedlicher Systeme behandelt. Zum einen ist dies ein großflächiges Tunnelelement mit dicker Barriere. Im Gegensatz zu Tunnelelementen mit $\mathrm{Al}_{2} \mathrm{O}_{3}$-Barriere, in denen nur einzelne Pinholes beobachtet wurden, bildeten sich hier durch Überspannung viele Pinholes mit einem charakteristischen Abstand von 50-100 nm zueinander. Dieses Verhalten konnte mit einem Modell erklärt werden, das die relativ große Zahl der Pinholes auf den deutlich niedrigeren Flächenwiderstand und damit auf die hohe Stromdichte in Tunnelelementen mit MgO-Barriere zurückführt. Bei dem zweiten System handelt es sich um ein nanostrukturiertes Tunnelelement mit ultradünner Barriere für Spinstrom induziertes Schalten. Bei diesen Elementen führt die sehr hohe Stromdichte von $9 \times 10^{7} \mathrm{~A} / \mathrm{cm}^{2}$ nach dem initialen Durchbruch zu einem massiven Materialtraltransport durch Elektromigration. Dadurch kann der Durchbruch weiter anwachsen und über das gesamte Element ausbreiten. 


\section{Literaturverzeichnis}

[1] Julliere, M.: Tunneling Between Ferromagnetic-Films. In: Physics Letters A 54 (1975), Nr. 3, S. 225-226

[2] Meservey, R. ; Tedrow, P. M. ; Fulde, P.: Magnetic Field Splitting Of Quasiparticle States In Superconducting Aluminum Films. In: Physical Review Letters 25 (1970), Nr. 18, S. 1270-\&

[3] Tedrow, P. M. ; Meservey, R.: Spin-Dependent Tunneling Into Ferromagnetic Nickel. In: Physical Review Letters 26 (1971), Nr. 4, S. 192-\&

[4] Baibich, M. N. ; Broto, J. M. ; Fert, A. ; Vandau, F. N. ; Petroff, F. ; Eitenne, P. ; Creuzet, G. ; Friederich, A. ; Chazelas, J.: Giant Magnetoresistance Of (001)Fe/(001) Cr Magnetic Superlattices. In: Physical Review Letters 61 (1988), November, Nr. 21, S. 2472-2475

[5] Binasch, G. ; Grünberg, P. ; Sauerbach, F. ; Zinn, W.: Enhanced Magnetoresistance In Layered Magnetic-Structures With Antiferromagnetic Interlayer Exchange. In: Physical Review B 39 (1989), März, Nr. 7, S. 48284830

[6] Miyazaki, T. ; Tezuka, N.: Giant Magnetic Tunneling Effect In Fe/Al2o3/Fe Junction. In: Journal Of Magnetism And Magnetic Materials 139 (1995), Januar, Nr. 3, S. L231-L234

[7] Moodera, J. S. ; Kinder, L. R. ; Wong, T. M. ; Meservey, R.: Large Magnetoresistance At Room-Temperature In Ferromagnetic Thin-Film Tunnel-Junctions. In: Physical Review Letters 74 (1995), April, Nr. 16, S. $3273-3276$

[8] Butler, W. H. ; Zhang, X. G. ; Schulthess, T. C. ; Maclaren, J. M.: Spin-dependent tunneling conductance of $\mathrm{Fe} / \mathrm{MgO} / \mathrm{Fe}$ sandwiches. In: Physical Review B 6305 (2001), Februar, Nr. 5, S. 054416

[9] Mathon, J. ; Umerski, A.: Theory of tunneling magnetoresistance of an epitaxial Fe/MgO/Fe(001) junction. In: Physical Review B 6322 (2001), Juni, Nr. 22, S. 220403 
[10] Bowen, M. ; Cros, V. ; Petroff, F. ; Fert, A. ; Boubeta, C. M. ; Costa-Kramer, J. L. ; Anguita, J. V. ; Cebollada, A. ; Briones, F. ; Teresa, J. M. ; Morellon, L. ; Ibarra, M. R. ; Guell, F. ; Peiro, F. ; Cornet, A.: Large magnetoresistance in $\mathrm{Fe} / \mathrm{MgO} / \mathrm{FeCo}(001)$ epitaxial tunnel junctions on GaAs(001). In: Applied Physics Letters 79 (2001), September, Nr. 11, S. 1655-1657

[11] Yuasa, S. ; Fukushima, A. ; Nagahama, T. ; Ando, K. ; Suzuki, Y.: High tunnel magnetoresistance at room temperature in fully epitaxial $\mathrm{Fe} / \mathrm{MgO} / \mathrm{Fe}$ tunnel junctions due to coherent spin-polarized Tunneling. In: Japanese Journal Of Applied Physics Part 2-Letters 63 Express Letters 43 (2004), April, Nr. 4B, S. L588-L590

[12] Yuasa, S. ; Nagahama, T. ; Fukushima, A. ; Suzuki, Y. ; Ando, K.: Giant room-temperature magnetoresistance in single-crystal $\mathrm{Fe} / \mathrm{MgO} / \mathrm{Fe}$ magnetic tunnel junctions. In: Nature Materials 3 (2004), Dezember, Nr. 12, S. 868-871

[13] Parkin, S. S. P. ; Kaiser, C. ; Panchula, A. ; Rice, P. M. ; Hughes, B. ; SAMANT, M. ; YANG, S. H.: Giant tunnelling magnetoresistance at room temperature with $\mathrm{MgO}$ (100) tunnel barriers. In: Nature Materials 3 (2004), Dezember, Nr. 12, S. 862-867

[14] Djayaprawira, D. D. ; Tsunekawa, K. ; Nagai, M. ; Maehara, H. ; Yamagata, S. ; Watanabe, N. ; Yuasa, S. ; Suzuki, Y. ; Ando, K.: 230tunnel junctions. In: Applied Physics Letters 86 (2005), Februar, Nr. 9, S. 092502

[15] Ikeda, S. ; Hayakawa, J. ; Ashizawa, Y. ; Lee, Y. M. ; Miura, K. ; Hasegawa, H. ; Tsunoda, M. ; Matsukura, F. ; Ohno, H.: Tunnel magnetoresistance of 604diffusion in $\mathrm{CoFeB} / \mathrm{MgO} / \mathrm{CoFeB}$ pseudo-spin-valves annealed at high temperature. In: Applied Physics Letters 93 (2008), August, Nr. 8, S. 082508

[16] Heiliger, C. ; Gradhand, M. ; Zahn, P. ; Mertig, I.: Tunneling magnetoresistance on the subnanometer scale. In: Physical Review Letters 99 (2007), August, Nr. 6, S. 066804

[17] Borgardt, N. I. ; Plikat, B. ; Seibt, M. ; Schroter, W.: Analysis of high resolution transmission electron microscope images of crystalline-amorphous interfaces. In: Ultramicroscopy 90 (2002), April, Nr. 4, S. 241-258 
[18] ThIEL, K.: Strukturelle Untersuchung der amorph/kristallinen Grenzfläche mittels quantitativer hochauflösender Transmissionselektronenmikroskopie an den Systemen $a-S i / c-S i$ und $a-G e / c-S i$, Universität Göttingen, Diss., 2006

[19] Himpsel, F. J. ; Ortega, J. E. ; Mankey, G. J. ; Willis, R. F.: Magnetic nanostructures. In: Advances In Physics 47 (1998), Juli, Nr. 4, S. 511-597

[20] IвACH, H. ; LÜTH, H.: Festkörperphysik. Springer, 1999

[21] Moodera, J. S. ; Nassar, J. ; Mathon, G.: Spin-tunneling in ferromagnetic junctions. In: Annual Review Of Materials Science 29 (1999), S. 381-432

[22] Stoner, E. C.: Collective electron ferromagnetism. In: Proc. Roy. Soc. A 165 (1938), S. $372-414$

[23] Meservey, R. ; Tedrow, P. M.: Spin-Polarized Electron-Tunneling. In: Physics Reports-Review Section Of Physics Letters 238 (1994), März, Nr. 4, S. $173-243$

[24] Monsma, D. J. ; Parkin, S. S. P.: Spin polarization of tunneling current from ferromagnet/Al2O3 interfaces using copper-doped aluminum superconducting films. In: Applied Physics Letters 77 (2000), Juli, Nr. 5, S. 720-722

[25] Moodera, J. S. ; Mathon, G.: Spin polarized tunneling in ferromagnetic junctions. In: Journal Of Magnetism And Magnetic Materials 200 (1999), Oktober, Nr. 1-3, S. 248-273

[26] Stearns, M. B.: Simple Explanation Of Tunneling Spin-Polarization Of Fe, Co, Ni And Its Alloys. In: Journal Of Magnetism And Magnetic Materials 5 (1977), Nr. 2, S. 167-171

[27] MÜnzenberg, M. ; Moodera, J. S.: Superconductor-ferromagnet tunneling measurements indicate sp-spin and d-spin currents. In: Physical Review B 70 (2004), August, Nr. 6, S. 060402

[28] Slonczewski, J. C.: Conductance And Exchange Coupling Of 2 Ferromagnets Separated By A Tunneling Barrier. In: Physical Review B 39 (1989), April, Nr. 10, S. 6995-7002

[29] Wulfhekel, W. ; Klaua, M. ; Ullmann, D. ; Zavaliche, F. ; Kirschner, J. ; Urban, R. ; Monchesky, T. ; Heinrich, B.: Single-crystal magnetotunnel junctions. In: Applied Physics Letters 78 (2001), Januar, Nr. 4, S. 509-511 
[30] Yuasa, S. ; Djayaprawira, D. D.: Giant tunnel magnetoresistance in magnetic tunnel junctions with a crystalline $\mathrm{MgO}(001)$ barrier. In: Journal Of Physics D-Applied Physics 40 (2007), November, Nr. 21, S. R337-R354

[31] Mavropoulos, P. ; Papanikolaou, N. ; Dederichs, P. H.: Complex band structure and tunneling through ferromagnet/insulator/ferromagnet junctions. In: Physical Review Letters 85 (2000), Juli, Nr. 5, S. 1088-1091

[32] Zhang, X. G. ; Butler, W. H.: Large magnetoresistance in bcc $\mathrm{Co} / \mathrm{MgO} / \mathrm{Co}$ and $\mathrm{FeCo} / \mathrm{MgO} / \mathrm{FeCo}$ tunnel junctions. In: Physical Review B 70 (2004), November, Nr. 17, S. 172407

[33] Klaua, M. ; Ullmann, D. ; Barthel, J. ; Wulfhekel, W. ; Kirschner, J. ; Urban, R. ; Monchesky, T. L. ; Enders, A. ; Cochran, J. F. ; HEINRICH, B.: Growth, structure, electronic, and magnetic properties of $\mathrm{MgO} / \mathrm{Fe}(001)$ bilayers and $\mathrm{Fe} / \mathrm{MgO} / \mathrm{Fe}(001)$ trilayers. In: Physical Review B 64 (2001), Oktober, Nr. 13, S. 134411

[34] Faure-Vincent, J. ; Tiusan, C. ; Jouguelet, E. ; Canet, F. ; Sajieddine, M. ; Bellouard, C. ; Popova, E. ; Hehn, M. ; Montaigne, F. ; SCHUHL, A.: High tunnel magnetoresistance in epitaxial $\mathrm{Fe} / \mathrm{MgO} / \mathrm{Fe}$ tunnel junctions. In: Applied Physics Letters 82 (2003), Juni, Nr. 25, S. 4507-4509

[35] Meyerheim, H. L. ; Popescu, R. ; Kirschner, J. ; Jedrecy, N. ; Sauvage-Simkin, M. ; Heinrich, B. ; Pinchaux, R.: Geometrical and compositional structure at metal-oxide interfaces: $\mathrm{MgO}$ on $\mathrm{Fe}(001)$. In: Physical Review Letters 87 (2001), August, Nr. 7, S. 076102

[36] Zhang, X. G. ; Butler, W. H. ; Bandyopadhyay, A.: Effects of the ironoxide layer in Fe-FeO-MgO-Fe tunneling junctions. In: Physical Review B 68 (2003), September, Nr. 9, S. 092402

[37] Heiliger, C. ; Zahn, P. ; Yavorsky, B. Y. ; Mertig, I.: Influence of the interface structure on the bias dependence of tunneling magnetoresistance. In: Physical Review B 72 (2005), November, Nr. 18, S. 180406

[38] Tiusan, C. ; Faure-Vincent, J. ; Bellouard, C. ; Hehn, M. ; JougueLET, E. ; SCHUHL, A.: Interfacial resonance state probed by spin-polarized tunneling in epitaxial Fe/MgO/Fe tunnel junctions. In: Physical Review Letters 93 (2004), September, Nr. 10, S. 106602

[39] Tusche, C. ; Meyerheim, H. L. ; Jedrecy, N. ; Renaud, G. ; Ernst, A. ; Henk, J. ; Bruno, P. ; KIrschner, J.: Oxygen-induced symmetrization 
and structural coherency in $\mathrm{Fe} / \mathrm{MgO} / \mathrm{Fe}(001)$ magnetic tunnel junctions. In: Physical Review Letters 95 (2005), Oktober, Nr. 17, S. 176101

[40] Yu, B. D. ; Kim, J. S.: Ab initio study of ultrathin MgO films on Fe(001): Influence of interfacial structures. In: Physical Review B 73 (2006), März, Nr. 12, S. 125408

[41] Gradhand, M. ; Heiliger, C. ; Zahn, P. ; Mertig, I.: Tunneling magnetoresistance with amorphous electrodes. In: Physical Review B 77 (2008), April, Nr. 13, S. 134403

[42] Miao, G. X. ; Park, Y. J. ; Moodera, J. S. ; Seibt, M. ; Eilers, G. ; MunzenBerG, M.: Disturbance of tunneling coherence by oxygen vacancy in epitaxial Fe/MgO/Fe magnetic tunnel junctions. In: Physical Review Letters 100 (2008), Juni, Nr. 24, S. 246803

[43] Mather, P. G. ; Read, J. C. ; Buhrman, R. A.: Disorder, defects, and band gaps in ultrathin (001) MgO tunnel barrier layers. In: Physical Review B 73 (2006), Mai, Nr. 20, S. 205412

[44] UbBen, K. U.: Strukturierung von $\mathrm{CoFeB} / \mathrm{MgO} / \mathrm{CoFeB}$ Tunnelmagnetowiderstands-Elementen, Universität Göttingen, Diplomarbeit, 2009

[45] Borgardt, N. I. ; Plikat, B. ; Schroter, W. ; Seibt, M. ; Wagner, T.: Atomic structure of the interface between silicon (111) and amorphous germanium. In: Physical Review B 70 (2004), November, Nr. 19, S. 195307

[46] Borgardt, N. I. ; Plikat, B. ; SeibT, M. ; Schroter, W.: The effect of the translational symmetry of crystalline silicon on the structure of amorphous germanium in the interfacial region. In: Crystallography Reports 49 (2004), März, Nr. 2, S. 225-232

[47] PliKAT, B.: Hochauflösende Transmissionselektronenmikroskopie an den kristallinamorphen Grenzflächen $c-S i / a-G e$ und c-Si/a-SiO2, Universität Göttingen, Diss., 1998

[48] Plikat, B. ; Borgardt, N. I. ; Seibt, M. ; Wagner, T. ; Schroter, W.: The modified structure of amorphous Ge near $\mathrm{Si}(111)$ substrates, Iop Publishing Ltd, 1999, S. 43-46

[49] Lynch, D. F. ; O’Keefe, M. A.: n-Beam Lattice Images. II. Methods Of calculation. In: Acta Crystallographica Section A A 28 (1972), Nr. NOV1, S. $536-548$ 
[50] IshizukA, K. ; UyedA, N.: New Theoretical And Practical Approach To Multislice Method. In: Acta Crystallographica Section A 33 (1977), Nr. SEP1, S. $740-749$

[51] Goodman, P. ; Moodie, A. F.: Numerical Evaluation Of N-Beam WaveFunctions In Electron-Scattering By Multi-Slice Method. In: Acta Crystallographica Section A A 30 (1974), Nr. MAR, S. 280-290

[52] Cowley, J. M. ; Moodie, A. F.: The Scattering Of Electrons By Atoms And Crystals .1. A New Theoretical Approach. In: Acta Crystallographica 10 (1957), Nr. 10, S. 609-619

[53] Stadelmann, P. A.: EMS - A Software Package For Electron-Diffraction Analysis And HREM: Image Simulation In Materials Science. In: Ultramicroscopy 21 (1987), Nr. 2, S. 131-145

[54] Howe, J. M.: Direct observation of order in the liquid at a solid-liquid interface by high-resolution transmission electron microscopy. In: Philosophical Magazine A-Physics Of Condensed Matter Structure Defects And Mechanical Properties 74 (1996), September, Nr. 3, S. 761-775

[55] Meyerheim, H. L. ; Popescu, R. ; Jedrecy, N. ; Vedpathak, M. ; Sauvage-Simkin, M. ; Pinchaux, R. ; Heinrich, B. ; Kirschner, J.: Surface x-ray diffraction analysis of the $\mathrm{MgO} / \mathrm{Fe}(001)$ interface: Evidence for an FeO layer. In: Physical Review B 65 (2002), April, Nr. 14, S. 144433

[56] WalteR, M.: Untersuchung der Transporteigenschaften von CoFeB $\mid \mathrm{MgO} / \mathrm{CoFeB}$ - Tunnelmagnetowiderstandselementen, Universität Göttingen, Diplomarbeit, 2009

[57] Eilers, G. ; Ulrichs, H. ; Munzenberg, M. ; Thomas, A. ; Thiel, K. ; SEIBT, M.: Long-range order on the atomic scale induced at CoFeB/MgO interfaces. In: Journal Of Applied Physics 105 (2009), April, Nr. 7, S. 073701

[58] Hyтch, M. J. ; Stobbs, W. M.: Quantitative Comparison Of HighResolution Tem Images With Image Simulations. In: Ultramicroscopy 53 (1994), März, Nr. 3, S. 191-203

[59] Hyтсн, M. J. ; Stobbs, W. M.: Quantitative Criteria For The Matching Of Simulations With Experimental Hrem Images. In: Microscopy Microanalysis Microstructures 5 (1994), April, Nr. 2, S. 133-151 
[60] Choi, Y. S. ; Tsunekawa, K. ; Nagamine, Y. ; Duayaprawira, D.: Transmission electron microscopy study on the polycrystalline Co$\mathrm{FeB} / \mathrm{MgO} / \mathrm{CoFeB}$ based magnetic tunnel junction showing a high tunneling magnetoresistance, predicted in single crystal magnetic tunnel junction. In: Journal Of Applied Physics 101 (2007), Januar, Nr. 1, S. 013907

[61] Lee, Y. M. ; Hayakawa, J. ; Ikeda, S. ; Matsukura, F. ; Ohno, H.: Effect of electrode composition on the tunnel magnetoresistance of pseudospin-valve magnetic tunnel junction with a $\mathrm{MgO}$ tunnel barrier. In: Applied Physics Letters 90 (2007), Mai, Nr. 21, S. 212507

[62] Mizuguchi, M. ; Suzuki, Y. ; Nagahama, T. ; Yuasa, S.: In situ scanning tunneling microscopy observations of polycrystalline $\mathrm{MgO}(001)$ tunneling barriers grown on amorphous CoFeB electrode. In: Applied Physics Letters 91 (2007), Juli, Nr. 1, S. 012507

[63] Thomas, A. ; Drewello, V. ; Schafers, M. ; Weddemann, A. ; Reiss, G. ; Eilers, G. ; Munzenberg, M. ; Thiel, K. ; Seibt, M.: Direct imaging of the structural change generated by dielectric breakdown in $\mathrm{MgO}$ based magnetic tunnel junctions. In: Applied Physics Letters 93 (2008), Oktober, Nr. 15, S. 152508

[64] Schäfers, M. ; Drewello, V. ; Reiss, G. ; Thomas, A. ; Thiel, K. ; Eilers, G. ; Münzenberg, M. ; Schuhmann, H. ; Seibt, M.: Electric breakdown in ultrathin $\mathrm{MgO}$ tunnel barrier junctions for spin-transfer torque switching. In: Applied Physics Letters 95 (2009), Nr. 23, S. 232119

[65] McPherson, J. W. ; Mogul, H. C.: Underlying physics of the thermochemical E model in describing low-field time-dependent dielectric breakdown in SiO2 thin films. In: Journal Of Applied Physics 84 (1998), August, Nr. 3, S. $1513-1523$

[66] Xi, H. W. ; Franzen, S. ; Guzman, J. I. ; MaO, S. I.: Degradation of magnetic tunneling junctions caused by pinhole formation and growth. In: Journal Of Magnetism And Magnetic Materials 319 (2007), Dezember, Nr. 1-2, S. 60-63

[67] Ho, P. S. ; Kwok, T.: Electromigration In Metals. In: Reports On Progress In Physics 52 (1989), März, Nr. 3, S. 301-348 



\section{Danksagung}

An dieser Stelle möchte ich mich bei all denen Bedanken, die zum Gelingen dieser Arbeit beigetragen haben.

Zuerst bei Prof. Dr. Markus Münzenberg dafür, dass er mir diese Arbeit ermöglicht hat. Sein mir entgegengebrachte Vertrauen und die Freiheiten, die er mir gelassen hat, weiß ich sehr zu schätzen.

Herrn Prof. Dr. Reiner Kirchheim danke ich für die Übernahme des Korreferats.

Karsten Thiel sei gedankt für die Einarbeitung in die Simulationssoftware und seine Diskussionsbereitschaft zu dem Thema. Außerdem danke ich ihm, wie auch Michael Seibt und Henning Schuhmann, für die vielen TEM-Aufnahmen.

Stellvertretend für die gesamte Arbeitsgruppe in Bielefeld bedanke ich mich bei Andy Thomas für die zur Verfügung gestellten Proben und für die gute Zusammenarbeit.

Für die immer angenehme Arbeitsatmosphäre gilt mein Dank meinen über die Zeit wechselnden Bürokollegen Jakob Walowski, Marvin Walter und Kai Ubben sowie allen anderen aktuellen und ehemaligen Mitgliedern der Arbeitsgruppe Münzenberg. Marvin Walter danke ich auch sehr herzlich für das gewissenhafte Korrekturlesen des Manuskripts.

Volker Radisch möchte ich für die Einweisung am FIB und seine guten Ratschläge bei Problemen oder bei der Umsetzung neuer Projekte danken.

Ein Dank geht auch an die Mitarbeiter der Werkstätten des IV. Physikalischen Instituts, ganz besonders an Andreas Juretzko, der mir bei all meinen Vorhaben mit Rat und Tat zur Seite stand.

Ich danke allen Mitgliedern des I. Physikalischen Instituts für die freundliche Aufnahme nach dem Umzug, insbesondere Katrin Gehrke für die Unterstützung bei der Einrichtung des neuen Labors. Ich habe mich vom ersten Tag an hier wohl gefühlt, wobei das Erste für mich auch vorher schon keine „Fremde Welt“ war. Für alle Mitglieder des IV. Instituts gilt das gleiche für die schöne Zeit vor dem Umzug.

Kai Gehrke gilt mein Dank für viele Disskussionen weit über die Physik hinaus, häufig bei Pizza und Bier im GroMo aber auch anderswo.

Bei meiner Familie und meinen Freunden möchte ich mich für die Unterstützung aus der Ferne auch herzlich bedanken. 



\section{Lebenslauf}

\section{Persönliche Daten}

Name: $\quad$ Gerrit Eilers

Geburtstag: $\quad$ 14.03.1977

Geburtsort: Westerstede

Staatsangehörigkeit: Deutsch

\section{Schulische Ausbildung}

1983 - 1987 Grundschule Bremer Heerstraße, Oldenburg

1987 - 1989 Orientierungsstufe Osternburg, Oldenburg

1889 - 1996 Altes Gymnasium, Oldenburg

\section{Wehrdienst}

09/1996 - 07/1997 Grundwehrdienst bei FlaRakGrp24, Oldenburg

\section{Hochschulausbildung}

WS 1997 Aufnahme des Studiums der Physik an der GeorgAugust-Universität Göttingen

01/2005 Diplom

Thema der Diplomarbeit: „Grundlegende Untersuchungen zu Tunnelmagnetowiderstandselementen: Schichtrauigkeit, Tunnelbarriere und mikromagnetische Simulationen“"

Seit 02/2005 Promotion an der Georg-August-Universität Göttingen 